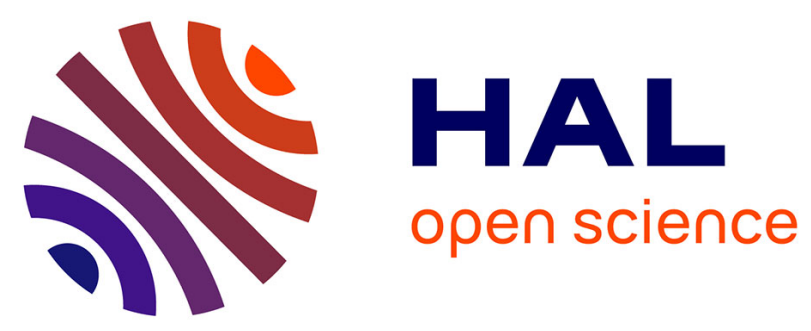

\title{
Internal wave generation by oscillation of a sphere, with application to internal tides
}

Bruno Voisin, Evgeny V. Ermanyuk, Jan-Bert Flór

\section{To cite this version:}

Bruno Voisin, Evgeny V. Ermanyuk, Jan-Bert Flór. Internal wave generation by oscillation of a sphere, with application to internal tides. Journal of Fluid Mechanics, 2011, 666, pp.308-357. 10.1017/S0022112010004209 . hal-00581790v2

\section{HAL Id: hal-00581790 \\ https://hal.science/hal-00581790v2}

Submitted on 17 May 2012

HAL is a multi-disciplinary open access archive for the deposit and dissemination of scientific research documents, whether they are published or not. The documents may come from teaching and research institutions in France or abroad, or from public or private research centers.
L'archive ouverte pluridisciplinaire HAL, est destinée au dépôt et à la diffusion de documents scientifiques de niveau recherche, publiés ou non, émanant des établissements d'enseignement et de recherche français ou étrangers, des laboratoires publics ou privés. 


\title{
Internal wave generation by oscillation of a sphere, with application to internal tides
}

\author{
B. VOISIN ${ }^{1} \dagger$, E. V. ERMANYUK ${ }^{2}$ AND J.-B. FLÓ $\mathbf{R}^{1}$ \\ ${ }^{1}$ Laboratoire des Écoulements Géophysiques et Industriels, CNRS - Université de Grenoble, BP 53, \\ 38041 Grenoble, France \\ ${ }^{2}$ Lavrentyev Institute of Hydrodynamics, Siberian Division of the Russian Academy of Science, \\ Prospekt Lavrentyev 15, Novosibirsk 630090, Russia
}

(Received 20 August 2009; revised 17 July 2010; accepted 4 August 2010; first published online 25 November 2010)

A joint theoretical and experimental study is performed on the generation of internal gravity waves by an oscillating sphere, as a paradigm of the generation of internal tides by barotropic tidal flow over three-dimensional supercritical topography. The theory is linear and three-dimensional, applies both near and far from the sphere, and takes into account viscosity and the unsteadiness arising from the interference with transients generated at the start-up. The waves propagate in conical beams, evolving with distance from a bimodal to unimodal wave profile. In the near field, the profile is asymmetric with its major peak towards the axis of the cones. The experiments involve horizontal oscillations and develop a cross-correlation technique for the measurement of the deformation of fluorescent dye planes to sub-pixel accuracy. At an oscillation amplitude of one fifth of the radius of the sphere, the waves are linear and the agreement between experiment and theory is excellent. As the amplitude increases to half the radius, nonlinear effects cause the wave amplitude to saturate at a value that is $20 \%$ lower than its linear estimate. Application of the theory to the conversion rate of barotropic tidal energy into internal tides confirms the expected scaling for flat topography, and shows its transformation for hemispherical topography. In the ocean, viscous and unsteady effects have an essentially local role, in keeping the wave amplitude finite at the edges of the beams, and otherwise dissipate energy on such large distances that they hardly induce any decay.

Key words: baroclinic flows, internal waves, topographic effects

\section{Introduction}

Internal, or baroclinic, tides are a special form of internal gravity waves generated in the ocean by ebb and flow of the surface, or barotropic, tide over seafloor topography. Internal tides are thought to extract energy from the barotropic tide at a global rate of about $1 \mathrm{TW}$ over the world's oceans, that is, one fourth of the total dissipation of the barotropic tide, and to transfer this energy to abyssal mixing through their selfinteraction, their interaction with the mean flow and their reflection at the seafloor, all of which may lead to breaking, local mixing events and the deposit of momentum 
(Garrett 2003). The large number of reviews devoted to these topics attest to the current interest in internal tides (see e.g. St. Laurent \& Garrett 2002; Kunze \& Llewellyn Smith 2004; Wunsch \& Ferrari 2004; Nycander 2005; Ivey, Winters \& Koseff 2008; Ferrari \& Wunsch 2009). Specific reviews have dealt with the theories of internal tide generation (Vlasenko, Stashchuk \& Hutter 2005; Garrett \& Kunze 2007).

In the so-called 'acoustic' limit, namely at small barotropic excursion compared with the horizontal scale of the topography, Bell $(1975 a, b)$ has shown that the internal tide is a linear monochromatic internal wave, generated in the frame of reference moving with the barotropic tide by the small horizontal oscillations of the topography. The experiments by Baines \& Fang (1985), Gostiaux \& Dauxois (2007) and Zhang, King \& Swinney (2008) for continental slopes, and Peacock, Echeverri \& Balmforth (2008) and Echeverri et al. (2009) for ridges, have confirmed this theoretical analysis. They have pointed out the separation of two regimes, subcritical and supercritical, respectively, depending on whether the topographic slope is everywhere smaller or locally larger than the slope of the wave rays, which is set by the frequency. The internal tide is generated preferentially at the critical locations where the topography and the rays are tangent to each other; it is accordingly more pronounced in the supercritical case.

The semi-circular cylinder in two dimensions, and the hemisphere in three dimensions, have slopes varying continuously between $90^{\circ}$ at the bottom and $0^{\circ}$ at the summit, making them supercritical at all frequencies. This revived the interest in the classical problem of internal wave generation by oscillating cylinders and spheres, and motivated the laboratory experiments and numerical simulations by Zhang, King \& Swinney (2007) for the cylinder and King, Zhang \& Swinney (2009) for the sphere.

Early theoretical studies took into account either the size of the forcing but not the viscosity of the fluid (see Hurley 1969, 1972, 1997 and Appleby \& Crighton 1986 for the cylinder, and Hendershott 1969, Sarma \& Krishna 1972, Appleby \& Crighton 1987 and Voisin 1991 for the sphere), or viscosity but not the size of the forcing (see Thomas \& Stevenson 1972; Gordon \& Stevenson 1972). Using an approximate representation of the cylinder or sphere introduced by Gorodtsov \& Teodorovich (1982, 1986), Ivanov (1989) and Makarov, Neklyudov \& Chashechkin (1990) showed how the two approaches can be combined together. Later, Hurley \& Keady (1997) and Hurley \& Hood (2001) showed how the approximation can be avoided for the cylinder.

These theoretical developments have relied closely on the simultaneous developments of experimental techniques. Most techniques are two-dimensional in that the signal is integrated across the width of the tank. Accordingly, they have been applied primarily to the cylinder. Early techniques, either non-intrusive such as Schlieren visualization (Görtler 1943; Mowbray \& Rarity 1967), Schlieren interferometry (Peters 1985; Merzkirch \& Peters 1992), Moiré fringes (Ivanov 1988, 1989; Sakai 1990) and shearing interferometry (Makarov et al. 1990), or intrusive such as buoyant drop tracking (Thomas \& Stevenson 1972; Gordon \& Stevenson 1972) and particle streaks (Xu et al. 1997), required a lot of effort while yielding access to only a fairly modest portion of the wave field. The situation changed drastically with the advent of digital image processing. Two new measurement techniques have been introduced: synthetic Schlieren, developed by Sutherland et al. (1999) and Dalziel, Hughes \& Sutherland (2000), and applied by Sutherland et al. (1999, 2000), Sutherland \& Linden (2002), Ermanyuk \& Gavrilov (2005, 2008), Mercier, Garnier \& 
Dauxois (2008), Thomas, Marino \& Dalziel (2009) and Clark \& Sutherland (2009); and particle image velocimetry (see e.g. Westerweel 1997), applied by Zhang et al. (2007).

The three-dimensionality of the sphere complicates the measurements. With particle streaks (McLaren et al. 1973; Lin, Boyer \& Fernando 1994) and particle image velocimetry (King et al. 2009), a light sheet allows the selection of a given plane of fluid. With Schlieren techniques, one possibility is to consider the axisymmetric problem of vertical oscillations, and to either use the integrated image directly, as did Peacock \& Weidman (2005), Chashechkin \& Prikhod'ko (2007) and Chashechkin (2007), or apply a simplified inverse tomographic transform developed by Onu, Flynn \& Sutherland (2003) and Flynn, Onu \& Sutherland (2003), as did Flynn et al. (2003) and Sutherland, Flynn \& Onu (2003). For non-axisymmetric problems, the full inverse tomographic transform has been developed by Décamp, Kozack \& Sutherland (2008), but remains to be applied.

The present paper considers the generation of internal gravity waves by an oscillating sphere both theoretically and experimentally. The theory is linear and based on Voisin (2003). It provides a counterpart for the sphere of the theory of Hurley (1997) and Hurley \& Keady (1997) for the cylinder. The solution satisfies the free-slip condition at the sphere exactly, and applies at arbitrary distance from it. The effect of viscosity on the propagation of the waves, and the effect of unsteadiness, namely the interference with transients generated at the start-up, are both accounted for. Only the physical implications of the theory are presented, while the details of the derivations will be reported elsewhere (Voisin 2010a,b,c).

The experiments involve horizontal oscillations of the sphere. The advantages of this configuration over vertical oscillations are twofold: conceptually the problem becomes relevant to internal tides; and practically the wave field becomes directional, such that energy is radiated parallel to the direction of oscillation, allowing absorption at the tank walls perpendicular to this direction while the other two walls remain available for visualization. Following Flynn et al. (2003), two spheres are used, one large and the other small, so as to create bimodal waves governed primarily by the size of the sphere and unimodal waves governed primarily by viscosity, respectively. For the latter, two amplitudes of oscillation are considered, so as to create linear and slightly nonlinear waves. The frequency of oscillation is larger than half the buoyancy frequency, precluding the propagation of higher harmonics.

A new simple technique is developed for measuring isopycnal displacements, based on the deformation of fluorescent dye planes. This technique has been introduced by Hopfinger et al. (1991) for the qualitative visualization of lee waves, and applied later by Bonnier, Bonneton \& Eiff (1998) to the qualitative visualization of stratified wakes and by Flór, Ungarish \& Bush (2002) and Flór, Bush \& Ungarish (2004) to the quantitative manual measurement of isopycnal displacements in spin-up flows. Here the measurement is made automatic, by developing an algorithm to process the experimental dye lines and determine their displacement to sub-pixel accuracy. Horizontal dye planes are used, illuminated by a vertical light sheet parallel to the direction of oscillation of the sphere. The sheet is able to scan the tank at time intervals small compared with the period of oscillation, allowing spatial reconstruction of the three-dimensional density field. We focus on the structure of the waves in the vertical plane through the centre of the sphere along its direction of oscillation. The structure of the waves in perpendicular planes will be considered in a separate paper (Ermanyuk, Flór \& Voisin 2010), together with the generation of higher harmonics. 
The paper is organized as follows. Section 2 introduces the theoretical expression of the waves and $\S 3$ analyses their structure. Section 4 discusses the relation of these results to the literature. The experimental set-up and data processing are described in $\S 5$, and the processed data are compared with theory in $\S 6$. Section 7 discusses the application of the theory to internal tides, with emphasis on the conversion rate of barotropic tidal energy into internal tides, for hemispherical topography and for two flat bell-shaped topographies. The main findings of the paper are summarized in $\S 8$, and the Appendix focuses on the emergence of the singularities of the wave field. Throughout the paper a number of one-sided Fourier transforms are used silently in the theory; they are taken from table 5 of Voisin (2003).

\section{Wave calculation}

\subsection{General case}

In an unbounded viscous uniformly stratified Boussinesq fluid of kinematic viscosity $v$ and buoyancy frequency $N$, a source of mass starts at time $t=0$ to release at the frequency $\omega<N$ the volume $q(\boldsymbol{x}) \exp (-\mathrm{i} \omega t)$ of fluid per unit volume per unit time. The source is characterized by its radius $a$ and the amplitude $A$ of the oscillations of the released fluid, such that $q(\boldsymbol{x})$ is a function of order $\omega A / a$ inside a region of radius $a$ around the origin, vanishing rapidly outside this region. The amplitude is assumed much smaller than the radius, a condition expressed as $K e \ll 1$ in terms of the amplitude ratio or the Keulegan-Carpenter number

$$
K e=\frac{A}{a},
$$

corresponding to the 'acoustic' limit of internal tides (Bell 1975a,b) and allowing the linearization of the equations of motion. The hydrostatic approximation, which would restrict the frequency range to $\omega \ll N$, is not made. The radiation condition is applied in its causal formulation, by adding to the real frequency an infinitesimal positive imaginary part; namely, whenever necessary in order to set the determination of multivalued functions of frequency, $\omega$ is replaced by $\omega+\mathrm{i} 0=\lim _{\epsilon \rightarrow 0}(\omega+\mathrm{i} \epsilon)$ with $\epsilon>0$. This procedure, discussed e.g. by Lighthill $(1978, \S 4.9)$, amounts to assuming that the source has grown up slowly as $\mathrm{e}^{\epsilon t}$ from zero at $t=-\infty$ to its present level.

Internal waves are generated. The geometry of steady inviscid waves is well-known (see e.g. Lighthill 1978, §4.4) and has been described for a sphere by Hendershott (1969), Appleby \& Crighton (1987) and Voisin (1991). It is illustrated in figure 1 for a sphere of radius $a$. The waves, which propagate at the angle $\theta=\arccos (\omega / N)$ to the vertical, are confined within a conical shell of thickness $2 a$ delimited by the two double cones of vertical axis and semi-angle $\theta$ tangent to the sphere above and below. Taking the $z$-axis along the upward vertical and denoting a horizontal component by a subscript $h$, we introduce conical polar coordinates $\left(x_{ \pm}, \varphi, z_{ \pm}\right)$in terms of the cylindrical polar coordinates $\left(r_{h}, \varphi, z\right)$ through

$$
x_{ \pm}=r_{h} \cos \theta \mp z \sin \theta, \quad z_{ \pm}= \pm r_{h} \sin \theta+z \cos \theta .
$$

The part of the shell situated between the two upper cones $x_{+}=-a$ and $x_{+}=a$ defines an upper wave beam, and the part of the shell situated between the two lower cones $x_{-}=-a$ and $x_{-}=a$ defines a lower wave beam. Within them, the phase propagates along the transverse coordinate $x_{ \pm}$with wavenumber vector $\left(k_{ \pm}>0, \varphi_{k}, m_{ \pm}=0\right)$, while energy propagates along the longitudinal coordinate $\pm z_{ \pm}$with group velocity vector $\left(0, \varphi_{k}, \pm \omega \tan \theta / k_{ \pm}\right)$. 


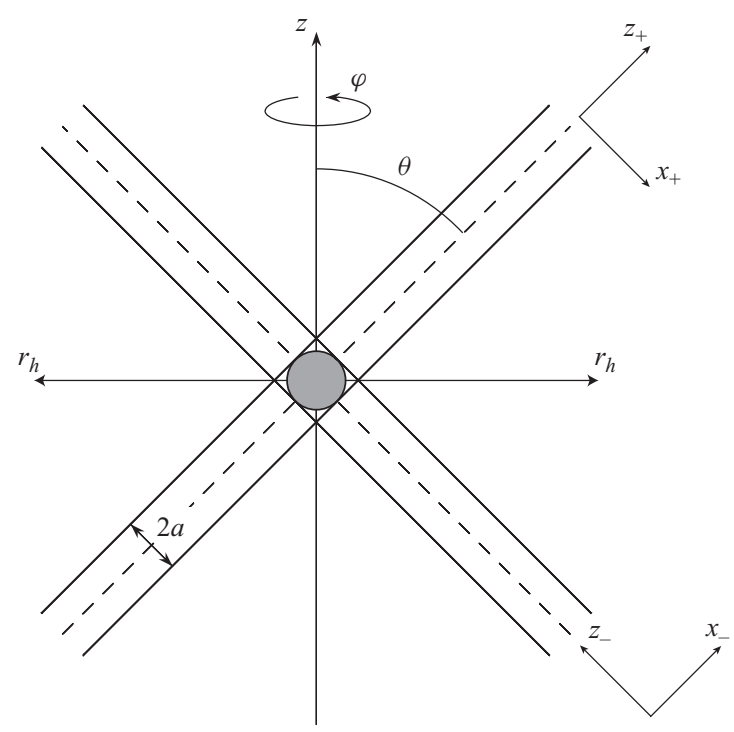

FIGURE 1. Coordinate systems for monochromatic internal waves and steady inviscid beam geometry for an oscillating sphere.

When unsteadiness and viscosity come into play, the structure of the beams is modified. It is governed by two dimensionless parameters: the product $\omega t$ characterizing the number of periods elapsed since the start-up, and the ReynoldsStokes number

$$
R e=\frac{2 \omega a^{2}}{v},
$$

characterizing, for an oscillating body, the ratio of its radius $a$ to the thickness $(2 v / \omega)^{1 / 2}$ of the boundary layer at its surface (see e.g. Batchelor 1967, §5.13). In the following, both parameters are assumed large, such that $\omega t \gg 1$ and $R e \gg 1$, allowing steady inviscid waves to form at the source before being affected by unsteadiness and viscosity as they propagate away. In particular, the boundary condition at the surface of an oscillating body is approximately steady and inviscid (see Hurley \& Hood 2001 for a flat plate and Davis \& Llewellyn Smith 2010 for a circular disk).

With no further assumption, Voisin (2003) derived expressions of the waves as spectral integrals in which the contribution of each wavenumber is determined by either of three mechanisms acting in isolation: the structure of the source through its spectrum

$$
q_{ \pm}\left(k_{ \pm}, \varphi_{k}, m_{ \pm}\right)=q(\boldsymbol{k})=\int q(\boldsymbol{x}) \exp (-\mathrm{i} \boldsymbol{k} \cdot \boldsymbol{x}) \mathrm{d}^{3} x ;
$$

unsteadiness through cutoff at a distance $\left|z_{ \pm}\right|$at the wavenumber $1 /\left(\alpha\left|z_{ \pm}\right|\right)$with

$$
\alpha=\frac{1}{\omega t \tan \theta}
$$

and viscosity through attenuation at the rate $\beta k_{ \pm}^{3}$ per unit distance along rays (Lighthill 1978, §§ 4.7 and 4.10) with

$$
\beta=\frac{v}{2 \omega \tan \theta} .
$$


Combining the three expressions, (4.21), (5.12) and (6.5) in Voisin (2003) respectively, we obtain for the velocity

$$
\begin{aligned}
\boldsymbol{u}= \pm \frac{\cos \theta}{8 \pi^{2}} \exp & -\mathrm{i} \omega t) \int_{0}^{2 \pi} \mathrm{d} \varphi_{k} \boldsymbol{e}_{m_{ \pm}}\left(\varphi_{k}\right) \int_{0}^{\cos \theta /(\alpha \mid z))} \mathrm{d} k_{ \pm} \exp \left(-\beta k_{ \pm}^{3}|z| / \cos \theta\right) \\
& \times k_{ \pm} q_{ \pm}\left(k_{ \pm}, \varphi_{k}, 0\right) \exp \left\{\mathrm{i} k_{ \pm}\left[r_{h} \cos \theta \cos \left(\varphi_{k}-\varphi\right) \mp z \sin \theta\right]\right\},
\end{aligned}
$$

where the unit vector $\boldsymbol{e}_{m_{ \pm}}$is related to the unit vectors $\left(\boldsymbol{e}_{r_{h}}, \boldsymbol{e}_{\varphi}, \boldsymbol{e}_{z}\right)$ of the cylindrical polar coordinate system by

$$
\boldsymbol{e}_{m_{ \pm}}\left(\varphi_{k}\right)= \pm \boldsymbol{e}_{r_{h}} \sin \theta \cos \left(\varphi_{k}-\varphi\right) \pm \boldsymbol{e}_{\varphi} \sin \theta \sin \left(\varphi_{k}-\varphi\right)+\boldsymbol{e}_{z} \cos \theta .
$$

The upper sign is used in the upper half-space and the lower sign in the lower halfspace, so that $\pm=\operatorname{sign} z$. The physical interpretation of this result is the superposition of the contributions of all the wavenumber vectors $\left(k_{ \pm}, \varphi_{k}, 0\right)$ satisfying the dispersion relation $m_{ \pm}=0$, subject to the radiation condition $k_{ \pm}>0$ of no waves coming in from infinity with vertical group velocity $\pm \omega \sin \theta / k_{ \pm}$opposite in sign to $z$. Its mathematical derivation is intricate and will be reported elsewhere (Voisin 2010c).

As the waves propagate further away from their source, the longitudinal coordinate becomes large, such that $\left|z_{ \pm}\right| / a \gg 1$, while the transverse coordinate remains moderate, such that $\left|x_{ \pm}\right| / a=O(1)$. The far field is reached, where the velocity becomes longitudinal and given by

$$
\begin{aligned}
& \boldsymbol{u} \sim \pm \frac{\cot ^{1 / 2} \theta}{2^{5 / 2} \pi^{3 / 2}} \boldsymbol{e}_{z_{ \pm}} \frac{\exp [-\mathrm{i}(\omega t+\pi / 4)]}{\left|z_{ \pm}\right|^{1 / 2}} \int_{0}^{1 /\left(\alpha \mid z_{ \pm}\right)} \mathrm{d} k_{ \pm} \exp \left(-\beta k_{ \pm}^{3}\left|z_{ \pm}\right|\right) \\
& \times k_{ \pm}^{1 / 2} q_{ \pm}\left(k_{ \pm}, \varphi, 0\right) \exp \left(\mathrm{i} k_{ \pm} x_{ \pm}\right),
\end{aligned}
$$

with $\boldsymbol{e}_{z_{+}}= \pm \boldsymbol{e}_{r_{h}} \sin \theta+\boldsymbol{e}_{z} \cos \theta$, as can be obtained formally from (2.7) by applying the method of stationary phase to the azimuthal integral and retaining the contribution of $\varphi_{k}=\varphi$ while discarding that of $\varphi_{k}=\varphi+\pi$. This corresponds physically to retaining only the wavenumber vectors $\left(k_{ \pm}>0, \varphi, 0\right)$ with group velocity vectors $\left(0, \varphi, \pm \omega \tan \theta / k_{ \pm}\right)$ pointing towards the observer.

In the laboratory, other quantities of interest are the vertical displacement $\zeta$, related to the vertical velocity $w=\partial \zeta / \partial t$, and the stability variation $\Delta N^{2}=-N^{2} \partial \zeta / \partial z$. Both follow from the vertical components of (2.7) and (2.9), by multiplying their integrands by $\mathrm{i} /(N \cos \theta)$ for $\zeta$ and $\mp N k_{ \pm} \tan \theta$ for $\Delta N^{2}$.

\subsection{Application to the sphere}

When the forcing is an oscillating body, at which surface the interaction with the fluid takes place, a natural approach is to replace the body by a distribution of singularities at this surface. The condition of continuity of the normal velocity at the surface becomes an integral equation for the distribution, hence the name boundary integral method (see e.g. Pozrikidis 1992, 2002). Gorodtsov \& Teodorovich (1982, 1986) pioneered its application to internal wave generation, for circular cylinders and spheres. However, they did not solve the proper integral equation and assumed instead that the distribution obtained for potential flow of a homogeneous fluid remains approximately valid for internal waves in a stratified fluid. Later, for twodimensional internal tides in an ocean of finite depth, Llewellyn Smith \& Young (2003) solved the integral equation analytically for a vertical barrier and Nycander 
(2006) for an infinite array of such barriers, but for more complicated topographies triangular, polynomial, sinusoidal and Gaussian ridges - Pétrélis, Llewellyn Smith \& Young (2006) and Balmforth \& Peacock (2009) had to resort to numerical procedures.

For the sphere, the integral equation can be solved analytically. Again, the derivation is intricate and will be reported elsewhere (Voisin 2010a). We consider the oscillations of a rigid sphere of radius $a$ at the frequency $\omega>N$ with velocity $\boldsymbol{U} \exp (-\mathrm{i} \omega t)$. The $y$-axis is taken perpendicular to the vertical plane of oscillation through the centre of the sphere. In Cartesian coordinates $(x, y, z)$, with $r=|\boldsymbol{x}|$ and $\boldsymbol{U}=(U, 0, W)$, the solution of the integral equation is

$$
q(x)=\left[\frac{2 U}{1+B(\omega / N)} \frac{x}{r}+\frac{W}{1-B(\omega / N)} \frac{z}{r}\right] \delta(r-a),
$$

and has the spectrum

$$
q(\boldsymbol{k})=-4 \mathrm{i} \pi a^{2}\left[\frac{2 U}{1+B(\omega / N)} \frac{k}{\kappa}+\frac{W}{1-B(\omega / N)} \frac{m}{\kappa}\right] j_{1}(\kappa a),
$$

where

$$
B\left(\frac{\omega}{N}\right)=\frac{\omega^{2}}{N^{2}}\left[1-\left(\frac{\omega^{2}}{N^{2}}-1\right)^{1 / 2} \arcsin \left(\frac{N}{\omega}\right)\right],
$$

$\delta(x)$ is the Dirac delta function, $\boldsymbol{k}=(k, l, m)$ and $\kappa=|\boldsymbol{k}|$. The notation $\mathrm{J}_{\mu}(x)$ will indicate a cylindrical Bessel function, and $j_{1}(x)=[\pi /(2 x)]^{1 / 2} \mathrm{~J}_{3 / 2}(x)=(\sin x) / x^{2}-$ $(\cos x) / x$ is a spherical Bessel function.

The frequency dependence of the solution expresses the effect of the stratification on the added mass of the sphere; in particular, it affects its dipole strength $\int \boldsymbol{x} q(\boldsymbol{x}) \mathrm{d}^{3} x$. This effect will be considered in detail in Voisin $(2010 b)$ and is consistent with the direct measurements of the added mass by Ermanyuk (2002) and Ermanyuk \& Gavrilov (2003). The limit $\omega / N \rightarrow \infty$ corresponds to oscillations too fast to be affected by buoyancy, so that $B(\omega / N) \rightarrow 1 / 3$ and the approximation of Gorodtsov \& Teodorovich $(1982,1986)$ is recovered, namely

$$
q(\boldsymbol{x})=\frac{3}{2} \boldsymbol{U} \cdot \frac{\boldsymbol{x}}{r} \delta(r-a) .
$$

At the frequencies $\omega<N$ of internal wave propagation, the determination of $B(\omega / N)$ follows from analytic continuation according to the radiation condition. We obtain

$$
B\left(\frac{\omega}{N}\right)=\frac{\omega^{2}}{N^{2}}\left\{1-\left(1-\frac{\omega^{2}}{N^{2}}\right)^{1 / 2}\left[\operatorname{arccosh}\left(\frac{N}{\omega}\right)+\mathrm{i} \frac{\pi}{2}\right]\right\},
$$

or, in terms of the angle of propagation $\theta=\arccos (\omega / N)$ to the vertical,

$$
B(\cos \theta)=\cos ^{2} \theta\left\{1-\sin \theta\left[\operatorname{arctanh}(\sin \theta)+\mathrm{i} \frac{1}{2} \pi\right]\right\},
$$

with variations illustrated in figure 2 .

In the following, the sphere oscillates at the frequency $\omega<N$ with amplitude $A \ll a$ at the angle $\eta$ to the horizontal. The position of its centre is $A(\cos \eta, 0, \sin \eta) \sin (\omega t+$ $\Theta)$, with $\Theta$ as the initial phase, so that for example $\eta=0$ and $\Theta=0$ correspond to horizontal oscillations started at $t=0$ from the centre of oscillation to the right. In complex notation we have $\boldsymbol{U}=\omega A(\cos \eta, 0, \sin \eta) \exp (-\mathrm{i} \Theta)$. Non-dimensionalizing the position as $\boldsymbol{X}=\boldsymbol{x} / a$, so that $\left(R_{h}, Z\right)=\left(r_{h}, z\right) / a$ and $\left(X_{ \pm}, Z_{ \pm}\right)=\left(x_{ \pm}, z_{ \pm}\right) / a$, and the 


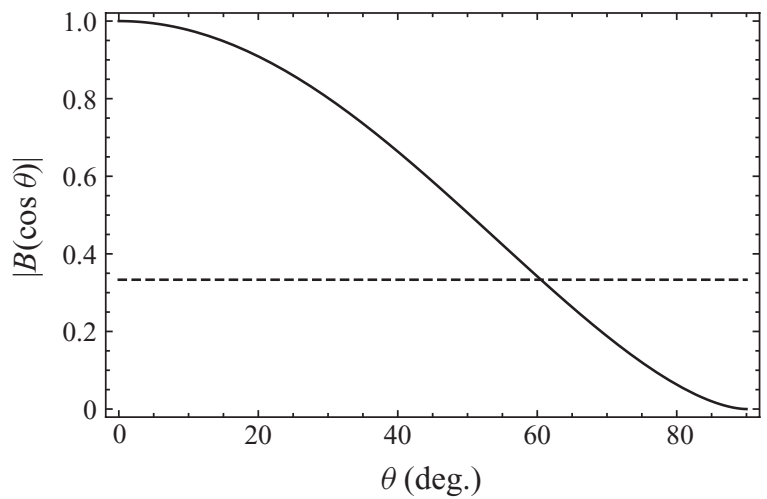

FIGURE 2. Variations of $|B(\cos \theta)|$ (solid line) and 'approximate' value $1 / 3$ in the absence of stratification (dashed line).

wavenumber vector as $\boldsymbol{K}=\boldsymbol{k} a$, so that $K_{ \pm}=k_{ \pm} a$, we obtain for the velocity, from (2.7) and (2.11),

$$
\begin{aligned}
\frac{u_{r_{h}}}{\omega A}= & \cos \theta \sin \theta \exp [-\mathrm{i}(\omega t+\Theta)] \int_{0}^{\cos \theta /(\alpha|Z|)} \mathrm{d} K_{ \pm} \exp \left(-\frac{\beta}{a^{2}} \frac{K_{ \pm}^{3}|Z|}{\cos \theta}\right) K_{ \pm} j_{1}\left(K_{ \pm}\right) \\
& \times\left\{\mathrm{i} \frac{2 \cos \theta \cos \eta}{1+B(\cos \theta)}\left[\frac{\mathrm{J}_{1}\left(K_{ \pm} R_{h} \cos \theta\right)}{K_{ \pm} R_{h} \cos \theta}-\mathrm{J}_{0}\left(K_{ \pm} R_{h} \cos \theta\right)\right] \cos \varphi\right. \\
& \left.\mp \frac{\sin \theta \sin \eta}{1-B(\cos \theta)} \mathrm{J}_{1}\left(K_{ \pm} R_{h} \cos \theta\right)\right\} \exp \left(-\mathrm{i} K_{ \pm}|Z| \sin \theta\right),
\end{aligned}
$$$$
\frac{u_{\varphi}}{\omega A}=\cos \theta \sin \theta \exp [-\mathrm{i}(\omega t+\Theta)] \int_{0}^{\cos \theta /(\alpha|Z|)} \mathrm{d} K_{ \pm} \exp \left(-\frac{\beta}{a^{2}} \frac{K_{ \pm}^{3}|Z|}{\cos \theta}\right) K_{ \pm} j_{1}\left(K_{ \pm}\right)
$$$$
\times \mathrm{i} \frac{2 \cos \theta \cos \eta}{1+B(\cos \theta)} \frac{\mathrm{J}_{1}\left(K_{ \pm} R_{h} \cos \theta\right)}{K_{ \pm} R_{h} \cos \theta} \sin \varphi \exp \left(-\mathrm{i} K_{ \pm}|Z| \sin \theta\right)
$$

$$
\begin{aligned}
\frac{w}{\omega A}= & \cos ^{2} \theta \exp [-\mathrm{i}(\omega t+\Theta)] \int_{0}^{\cos \theta /(\alpha|Z|)} \mathrm{d} K_{ \pm} \exp \left(-\frac{\beta}{a^{2}} \frac{K_{ \pm}^{3}|Z|}{\cos \theta}\right) K_{ \pm} j_{1}\left(K_{ \pm}\right) \\
& \times\left[ \pm \frac{2 \cos \theta \cos \eta}{1+B(\cos \theta)} \mathrm{J}_{1}\left(K_{ \pm} R_{h} \cos \theta\right) \cos \varphi+\mathrm{i} \frac{\sin \theta \sin \eta}{1-B(\cos \theta)} \mathrm{J}_{0}\left(K_{ \pm} R_{h} \cos \theta\right)\right] \\
& \times \exp \left(-\mathrm{i} K_{ \pm}|Z| \sin \theta\right) .
\end{aligned}
$$

The longitudinal component follows as $u_{z_{ \pm}}= \pm u_{r_{h}} \sin \theta+w \cos \theta$. In the far field, this component becomes dominant, according to

$$
\begin{aligned}
\frac{\boldsymbol{u}}{\omega A} \sim \frac{\cot ^{1 / 2} \theta}{2}[ \pm & \left.\frac{2 \cos \theta \cos \eta}{1+B(\cos \theta)} \cos \varphi-\frac{\sin \theta \sin \eta}{1-B(\cos \theta)}\right] \boldsymbol{e}_{z_{ \pm}} \frac{\exp [-\mathrm{i}(\omega t+\Theta+3 \pi / 4)]}{\left|Z_{ \pm}\right|^{1 / 2}} \\
& \times \int_{0}^{1 /\left(\alpha\left|Z_{ \pm}\right|\right)} \exp \left(-\frac{\beta}{a^{2}} K_{ \pm}^{3}\left|Z_{ \pm}\right|\right) \mathrm{J}_{3 / 2}\left(K_{ \pm}\right) \exp \left(\mathrm{i} K_{ \pm} X_{ \pm}\right) \mathrm{d} K_{ \pm}
\end{aligned}
$$

either from (2.9) and (2.11), or from replacing the Bessel functions $\mathrm{J}_{0}$ and $\mathrm{J}_{1}$ in (2.16) by their asymptotic expansions and retaining the outward terms varying as $\exp \left(\mathrm{i} K_{ \pm} R_{h} \cos \theta\right)$ while discarding the inward terms varying as $\exp \left(-\mathrm{i} K_{ \pm} R_{h} \cos \theta\right)$. 
The normalized vertical displacement $\zeta / A$ and the stability variation $\left(\Delta N^{2} / N^{2}\right)(a / A)$ follow from the normalized vertical velocity $w /(\omega A)$ by multiplying its integrand by $\mathrm{i}$ and $\mp K_{ \pm} \sin \theta$, respectively.

\section{Wave structure}

Owing to the assumptions of large times $\omega t \gg 1$ and low viscosity $R e \gg 1$, the wave field is built by small unsteady and viscous effects around a steady inviscid skeleton. We investigate the interplay of these effects separately in the near field, corresponding to moderate propagation distances $\left|Z_{ \pm}\right|=O(1)$, and in the far field, corresponding to large propagation distances $\left|Z_{ \pm}\right| \gg 1$. The discussion is expressed in terms of the vertical displacement, which is the quantity measured in the present experiments.

\subsection{Near field}

Setting $\alpha=0$ and $\beta=0$ in (2.16c), and denoting henceforth a steady inviscid result by a subscript 0 , we obtain

$$
\begin{aligned}
\frac{\zeta_{0}}{A}= & \cos ^{2} \theta \exp [-\mathrm{i}(\omega t+\Theta)]\left\{ \pm \frac{2 \cos \theta \cos \eta}{1+B(\cos \theta)}\left[\left(X_{+}+1\right)^{1 / 2}\left(X_{-}-1\right)^{1 / 2}\right.\right. \\
& -\left(X_{+}-1\right)^{1 / 2}\left(X_{-}+1\right)^{1 / 2}+\frac{1}{2}\left(\frac{X_{+}+1}{X_{-}-1}\right)^{1 / 2}-\frac{1}{2}\left(\frac{X_{-}-1}{X_{+}+1}\right)^{1 / 2} \\
& \left.+\frac{1}{2}\left(\frac{X_{+}-1}{X_{-}+1}\right)^{1 / 2}-\frac{1}{2}\left(\frac{X_{-}+1}{X_{+}-1}\right)^{1 / 2}\right] \frac{\cos \varphi}{X_{+}+X_{-}} \\
& +\frac{\sin \theta \sin \eta}{1-B(\cos \theta)}\left[\mathrm{i} \ln \frac{\left(X_{+}+1\right)^{1 / 2}-\mathrm{i}\left(X_{-}-1\right)^{1 / 2}}{\left(X_{+}-1\right)^{1 / 2}-\mathrm{i}\left(X_{-}+1\right)^{1 / 2}}\right. \\
& \left.\left.+\frac{1}{2\left(X_{+}+1\right)^{1 / 2}\left(X_{-}-1\right)^{1 / 2}}+\frac{1}{2\left(X_{+}-1\right)^{1 / 2}\left(X_{-}+1\right)^{1 / 2}}\right]\right\}
\end{aligned}
$$

where $X_{+}$must be understood as $X_{+}+\mathrm{i} 0 \operatorname{sign} Z$ and $X_{-}$as $X_{-}-\mathrm{i} 0 \operatorname{sign} Z$. This result, and the similar result for a pulsating sphere of the radial velocity $U \exp (-\mathrm{i} \omega t)$ represented by the source $q(x)=U \delta(r-a)$, are equivalent to those of Hendershott (1969), Sarma \& Krishna (1972), Appleby \& Crighton (1987) and Voisin (1991). They are the analogues for the sphere of the result of Hurley (1997) for a horizontal circular cylinder oscillating perpendicular to its axis. Their complexity, compared with the simplicity of the latter, illustrates the difficulty of three-dimensional geometries.

The waves have singular amplitudes on the two critical double cones of vertical axis and semi-angle $\theta$ tangent to the sphere above and below, and phase variations in between, yielding the structure represented in figure 1: two wave beams are formed, an upper beam $\left|X_{+}\right|<1$ delimited by the two upper cones and a lower beam $\left|X_{-}\right|<1$ delimited by the two lower cones, with an integrable inverse square root singularity at the cones.

Of particular interest is the region, of biconical shape, with a close-up in figure 3, where the beams overlap. At the poles $Z= \pm \csc \theta$ and equator $R_{h}=\sec \theta$ of the bicone, the critical cones intersect and the order of the singularity may become higher. In order to determine it, we look at the waves on the polar axis and in the 


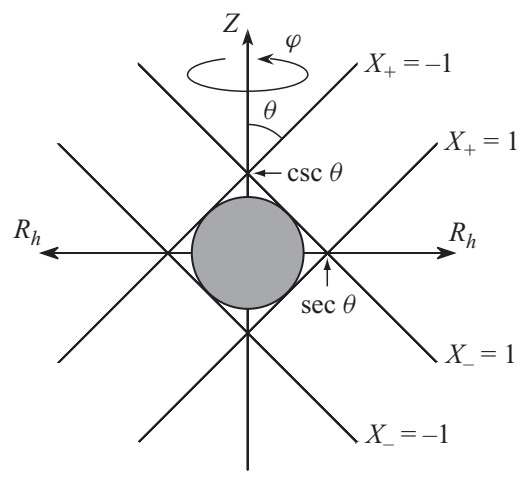

FIGURE 3. Geometry of the overlap region.

equatorial plane. On the polar axis $R_{h}=0$ we have

$$
\begin{array}{r}
\frac{\zeta_{0}}{A}=\mathrm{i} \cos ^{2} \theta \exp [-\mathrm{i}(\omega t+\Theta)] \frac{\sin \theta \sin \eta}{1-B(\cos \theta)}\left[\frac{|Z| \sin \theta}{1-Z^{2} \sin ^{2} \theta}+\operatorname{arctanh}(|Z| \sin \theta)+\mathrm{i} \frac{\pi}{2}\right] \\
\frac{(1<|Z|<\csc \theta), \quad(3.2 a)}{\frac{\zeta_{0}}{A}=\mathrm{i} \cos ^{2} \theta \exp [-\mathrm{i}(\omega t+\Theta)] \frac{\sin \theta \sin \eta}{1-B(\cos \theta)}\left[\frac{|Z| \sin \theta}{1-Z^{2} \sin ^{2} \theta}+\operatorname{arccoth}(|Z| \sin \theta)\right]} \\
(|Z|>\csc \theta), \quad(3.2 b)
\end{array}
$$

and as the equatorial plane is approached from above or below, namely as $Z \rightarrow \pm 0$, we have

$$
\begin{array}{r}
\frac{\zeta_{0}}{A}=\cos ^{2} \theta \exp [-\mathrm{i}(\omega t+\Theta)]\left[ \pm \mathrm{i} \frac{2 \cos \theta \cos \eta}{1+B(\cos \theta)} \frac{R_{h} \cos \theta \cos \varphi}{\left(1-R_{h}^{2} \cos ^{2} \theta\right)^{1 / 2}}-\frac{\sin \theta \sin \eta}{1-B(\cos \theta)} \frac{\pi}{2}\right] \\
\left(1<R_{h}<\sec \theta\right), \quad(3.3 a) \\
\frac{\zeta_{0}}{A}=\cos ^{2} \theta \exp [-\mathrm{i}(\omega t+\Theta)] \frac{\sin \theta \sin \eta}{1-B(\cos \theta)}\left[\frac{1}{\left(R_{h}^{2} \cos ^{2} \theta-1\right)^{1 / 2}}-\arcsin \left(\frac{1}{R_{h} \cos \theta}\right)\right] \\
\left(R_{h}>\sec \theta\right), \quad(3.3 b)
\end{array}
$$

with variations plotted in figure 4 . At the top and bottom of the sphere, namely at $\left(R_{h}=0,|Z|=1\right)$, we verify that $w_{0}=-\mathrm{i} \omega \zeta_{0}=\omega A \sin \eta \exp [-\mathrm{i}(\omega t+\Theta)]=W \exp (-\mathrm{i} \omega t)$, consistent with continuity of the prescribed normal velocity.

On the polar axis, no vertical motion takes place for horizontal oscillations of the sphere. For vertical oscillations, the phase jumps by $\pi$ at the poles of the bicone and the amplitude has an inverse first power singularity. As predicted by Appleby \& Crighton (1987), the two poles are higher-order focusing singularities at which the velocity is no longer integrable. Simakov (1994) and Bühler \& Muller (2007) have investigated the fate of these singularities in more complex geometries. The present analysis allows us to consider their regularization by unsteady and viscous effects. Coming back to $(2.16 c)$ and introducing parameters

$$
\tau_{u}=\frac{\sin \theta \cos \theta}{\alpha}=\omega t \sin ^{2} \theta, \quad \tau_{v}=\left(\frac{a^{2} \sin \theta \cos \theta}{\beta}\right)^{1 / 3}=\left(\operatorname{Re} \sin ^{2} \theta\right)^{1 / 3},
$$


(a)

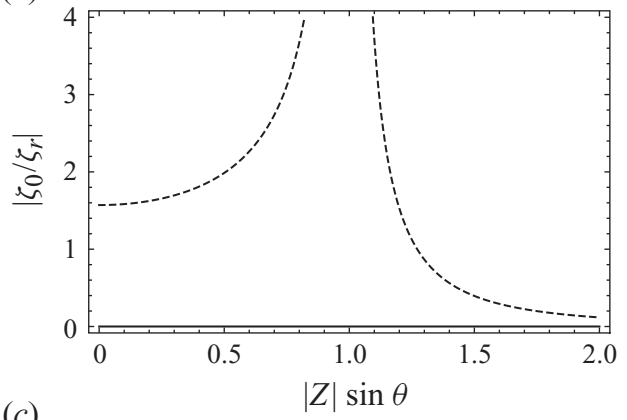

(c)

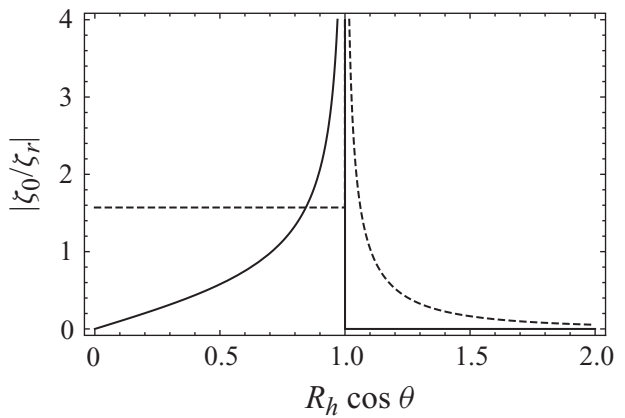

(b)

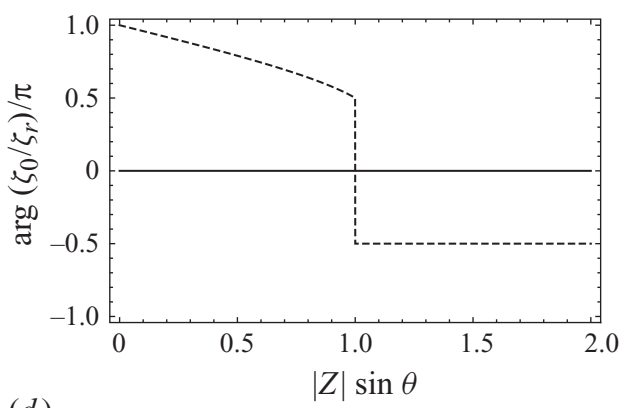

$(d)$

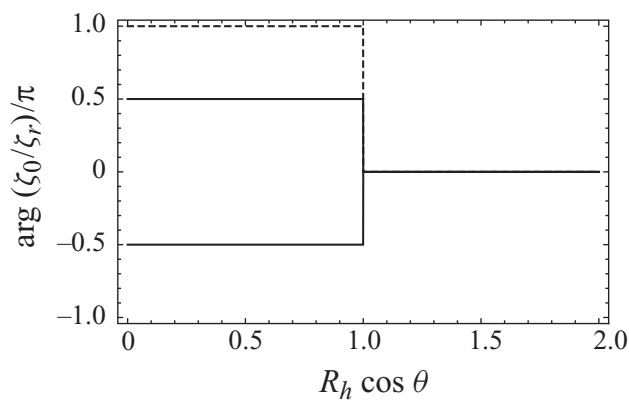

FIGURE 4. Variations of the steady inviscid vertical displacement in $(a, c)$ amplitude and $(b, d)$ phase on $(a, b)$ the polar axis $R_{h}=0$ and $(c, d)$ the equatorial plane $Z=0$, for a sphere oscillating horizontally (solid lines) or vertically (dashed lines). The displacement is normalized by $\zeta_{r}=2 A \exp [-\mathrm{i}(\omega t+\Theta)] \cos ^{3} \theta \cos \varphi /[1+B(\cos \theta)]$ for horizontal oscillations and $A \exp [-\mathrm{i}(\omega t+\Theta)] \cos ^{2} \theta \sin \theta /[1-B(\cos \theta)]$ for vertical oscillations.

we obtain at the foci

$$
\begin{aligned}
\frac{\zeta}{A}=-\cos ^{2} \theta \exp [-\mathrm{i}(\omega t & +\Theta)] \frac{\sin \theta \sin \eta}{1-B(\cos \theta)} \\
& \times \int_{0}^{\tau_{u}} \exp \left(-K_{ \pm}^{3} / \tau_{v}^{3}\right) K_{ \pm} j_{1}\left(K_{ \pm}\right) \exp \left(-\mathrm{i} K_{ \pm}\right) \mathrm{d} K_{ \pm} .
\end{aligned}
$$

The evolution of the amplitude with $\tau_{u}$ and $\tau_{v}$ is presented in figure 5. Figure 5(a), in particular, exhibits how the amplitude increases with time until it reaches a steady value at a time determined, together with the value itself, by the Reynolds-Stokes number. For the experiments of Flynn et al. (2003), involving a small sphere with $R e=350$ and a large sphere with $R e=1000$, oscillating vertically at $\omega / N=0.49$, the steady state is predicted to start at, say, $\tau_{u} \approx 9$ for the former and 13 for the latter, while the measurements were performed during the third period $4 \pi<\omega t<6 \pi$, that is, $9.5<\tau_{u}<14$. Accordingly, the waves were already steady at the foci for the small sphere and almost steady for the large sphere.

Caution must be exerted, in interpreting figure $5(a)$ and the forthcoming figures 14 and 16, that the present results, though starting from $\zeta=0$ at $t=0$ and hence physically plausible for all $t$, were obtained on the assumption $\omega t \gg 1$ and cannot hold for $\omega t \ll 1$. Being started up instantaneously from rest, the initial motion of the fluid is potential (see e.g. Batchelor 1967, §6.10, and Voisin 1991, Appendix B), implying that $\zeta$ varies as $t$ for $\omega t \ll 1$ whereas (3.5) varies as $t^{3}$ in the same limit. As a rule, we expect the present results to hold, say, for $\omega t \gtrsim 1$. 

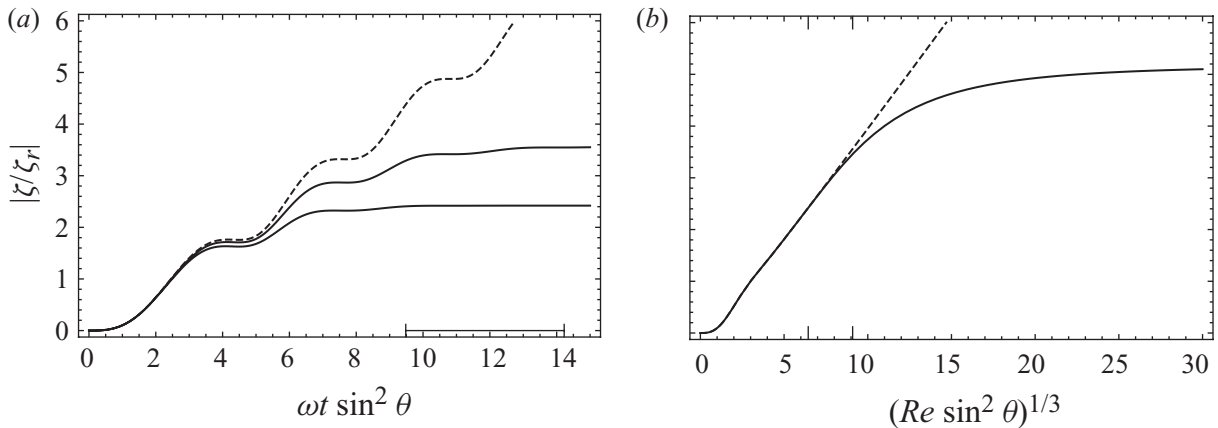

FiguRE 5. Evolution of the vertical displacement amplitude at the focusing singularities for a vertically oscillating sphere, with $(a)$ non-dimensional time $\omega t$ and $(b)$ Reynolds-Stokes number $R e$. In $(a)$ the solid lines are plotted for $R e=350$ (lower line) and 1000 (upper line), and the dashed line in the inviscid limit $R e \rightarrow \infty$; the horizontal segment corresponds to the interval $4 \pi<\omega t<6 \pi$ at $\omega / N=0.49$. In $(b)$ the solid line is plotted for $\omega t=5 \pi$, and the dashed line in the steady limit $\omega t \rightarrow \infty$; the bigger tick marks correspond to $R e=350$ and 1000 for $\omega / N=0.49$. The values of $R e, \omega t$ and $\omega / N$ are those for the small and large spheres in the experiments of Flynn et al. (2003).

In the equatorial plane, the phase jumps at the equator of the bicone and the amplitude has an inverse square root singularity. Accordingly, no higher-order singularity is observed. What makes this annular singularity remarkable, however, is that neither unsteady nor viscous effects affect it; there, nonlinear effects are likely to be significant, vitiating the wave/vortex decomposition on which the theory is based (Voisin 2003). In addition, for horizontal oscillations of the sphere, the phase jumps by $\pi$ as the equatorial disk $1<R_{h}<\sec \theta$ is traversed, implying that the upper and lower sides of the disk oscillate in opposition so that the velocity is discontinuous.

The evolution of the horizontal amplitude profiles with distance from the equatorial plane is illustrated in figure 6, calculated from (2.16c). Owing to unsteady and viscous effects, the annular singularity separates into mild humps along the two critical cones intersecting at the equator; one of the humps reaches the polar axis at the focusing singularity and is reflected away from it; then, together with the other hump it determines the edges of the wave beam. Figure 7 adds phase information to the picture, transforming the amplitude profiles into isopycnal lines.

In order to get better insight into the structure of the beam, selected profiles are plotted in figures 8 and 9 in the conditions of the experiments of $\S 6$, namely at the instant $\omega t=41 \pi$ of the horizontal oscillations at $\omega / N=0.74$ of two spheres, one large with $R e=1500$ and the other small with $R e=520$. For the large sphere, the two humps are asymmetrical, with a major hump near the polar axis and a minor hump away from it, yielding a double-peaked shape in which one may recognize the outline of a 'boa constrictor digesting an elephant' (Saint-Exupéry 1946). As the waves propagate away from the equatorial plane the profile becomes gradually symmetrical, until at, say, $|Z|=3$ the far field is reached, where a more appropriate description involves the transverse coordinate $X_{ \pm}$in which the profile is fully symmetrical. For the small sphere, the profiles are plotted further away and include a single hump at the centre of the beam, implying that the two humps have merged into one. The explanation for this phenomenon requires the consideration of the far field. 

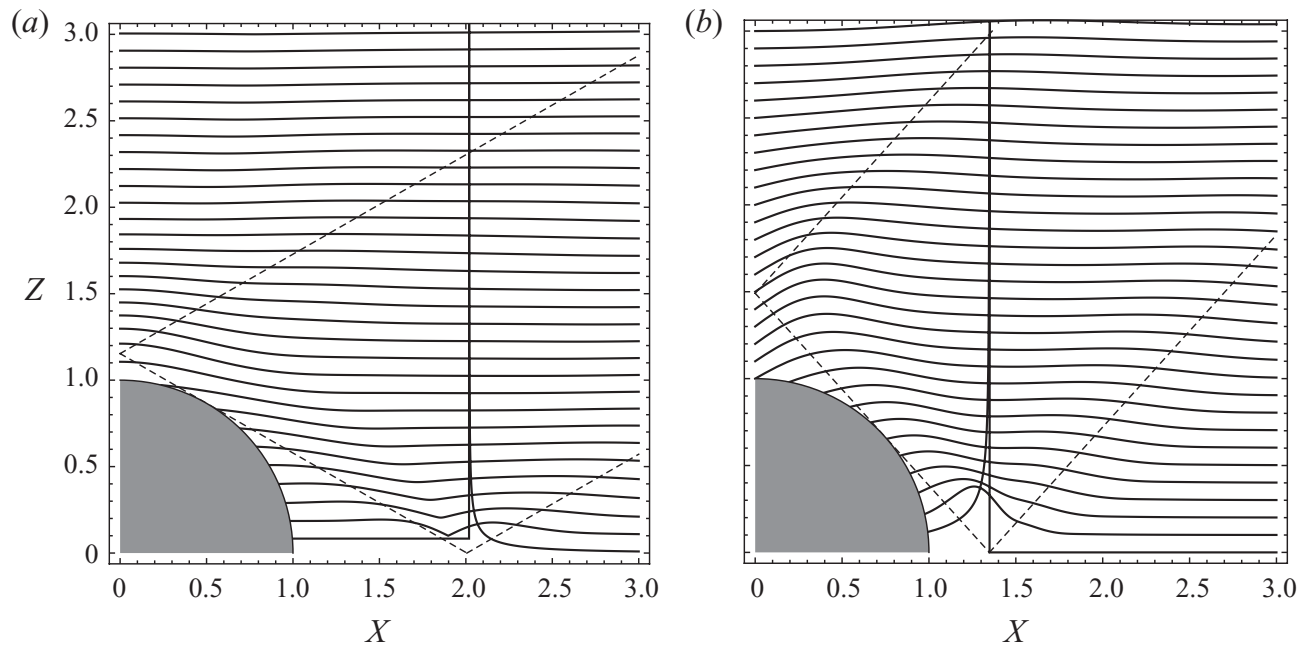

FIGURE 6. Horizontal profiles of the vertical displacement amplitude in the plane $Y=0$, for spheres oscillating (a) vertically at $\omega / N=0.49, R e=1000, \omega t=5 \pi$ and $K e=0.27$, corresponding to the large sphere in the experiments of Flynn et al. (2003), and (b) horizontally at $\omega / N=0.74, R e=1500, \omega t=41 \pi$ and $K e=0.18$, corresponding to the large sphere in the present experiments. The plotted quantity is $Z+|\zeta| / a$ at $Z=0,0.1,0.2, \ldots$, and the dashed lines represent the steady inviscid critical cones.
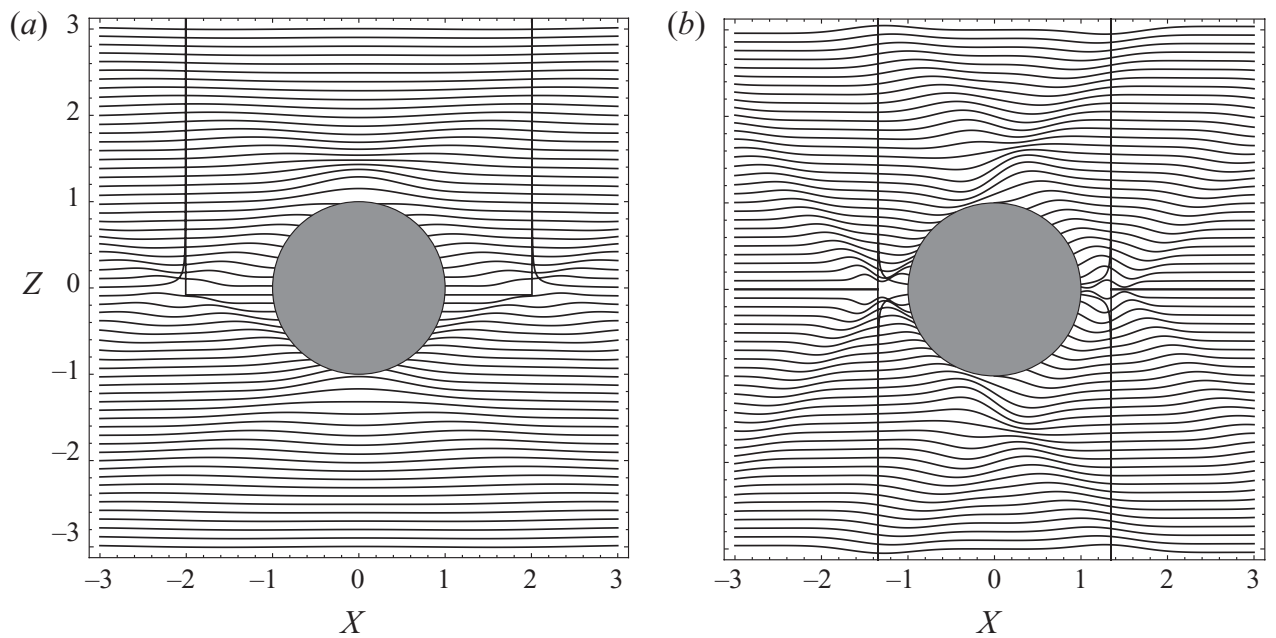

FIGURE 7. Isopycnal lines in the plane $Y=0$. The mode of representation is the same as in figure 6, except for the plotted quantity which is $Z+\zeta / a$, and the time in $(b)$ which is $\omega t=40 \pi+3.06$ as in figure 18. The initial phase is $\Theta=\pi$, corresponding to spheres starting at $t=0$ to move $(a)$ downwards and $(b)$ to the left from the centre of oscillation.

\subsection{Far field}

Either writing $X_{\mp} \sim\left|Z_{ \pm}\right| \sin (2 \theta) \gg 1$ and $\left|X_{ \pm}\right|=O(1)$ in (3.1), or setting $\alpha=0$ and $\beta=0$ in (2.17), the steady inviscid skeleton becomes, in the far field,

$$
\begin{aligned}
\frac{\zeta_{0}}{A} \sim \frac{\cos ^{3 / 2} \theta}{2^{5 / 2} \sin ^{1 / 2} \theta}\left[\mp \frac{2 \cos \theta \cos \eta}{1+}+B(\cos \theta)\right. & \left.\cos \varphi+\frac{\sin \theta \sin \eta}{1-B(\cos \theta)}\right] \\
& \times \frac{\exp [-\mathrm{i}(\omega t+\Theta)]}{\left|Z_{ \pm}\right|^{1 / 2}} \frac{\left[\left(X_{ \pm}+1\right)^{1 / 2}-\left(X_{ \pm}-1\right)^{1 / 2}\right]^{3}}{\left(X_{ \pm}+1\right)^{1 / 2}\left(X_{ \pm}-1\right)^{1 / 2}},
\end{aligned}
$$




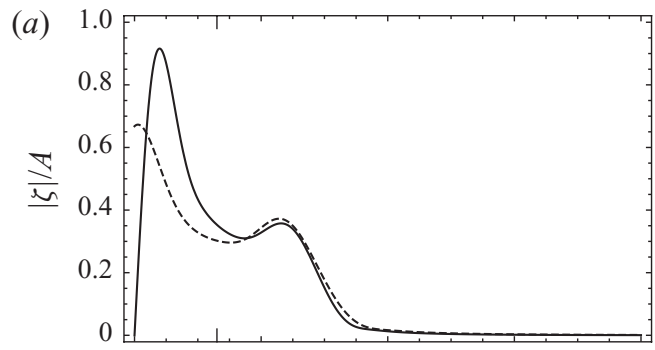

(b)
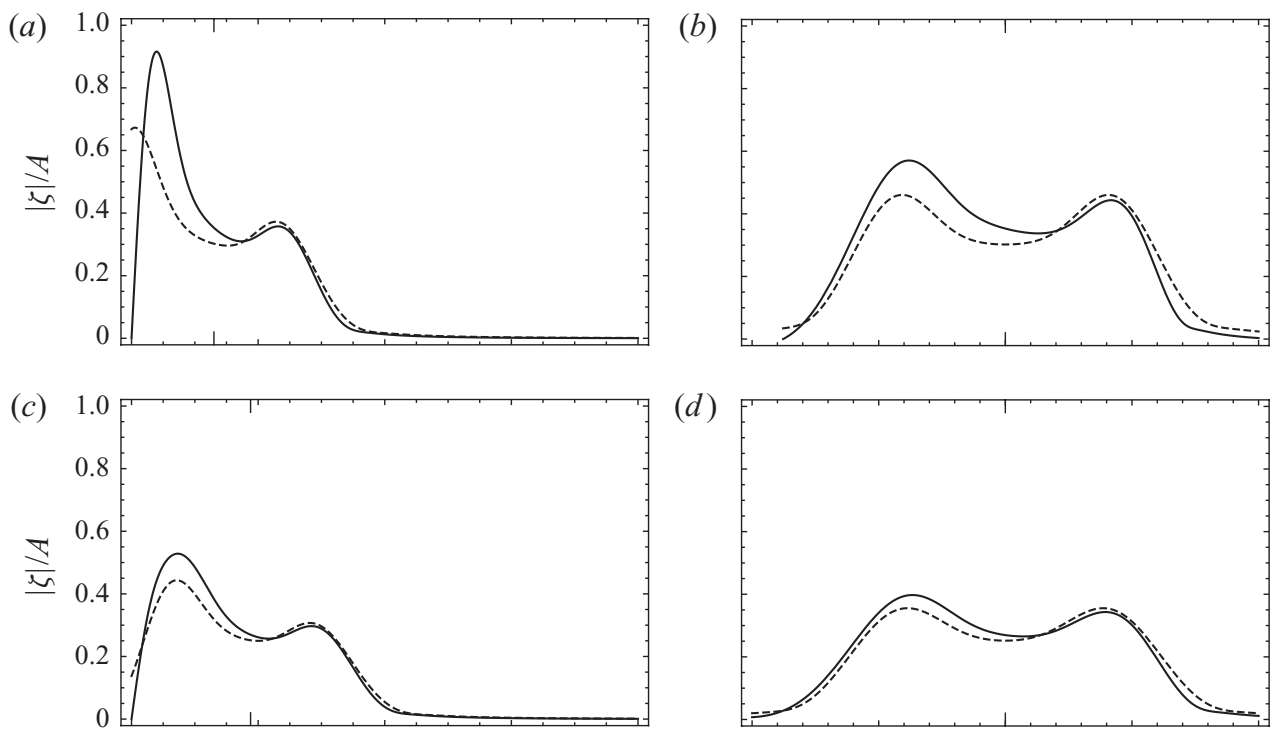

$(d)$

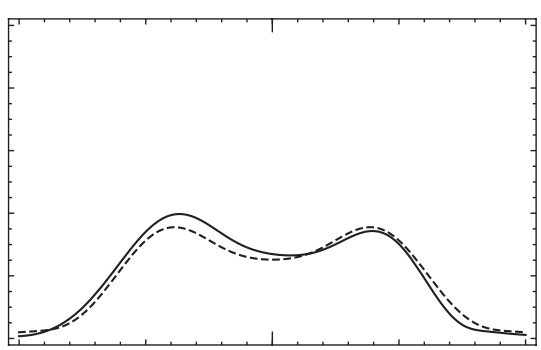

(e)

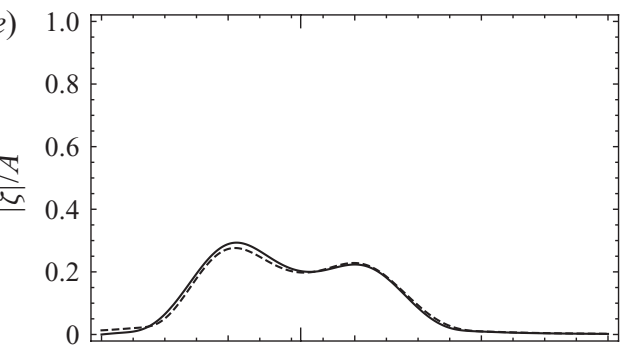

$(f)$
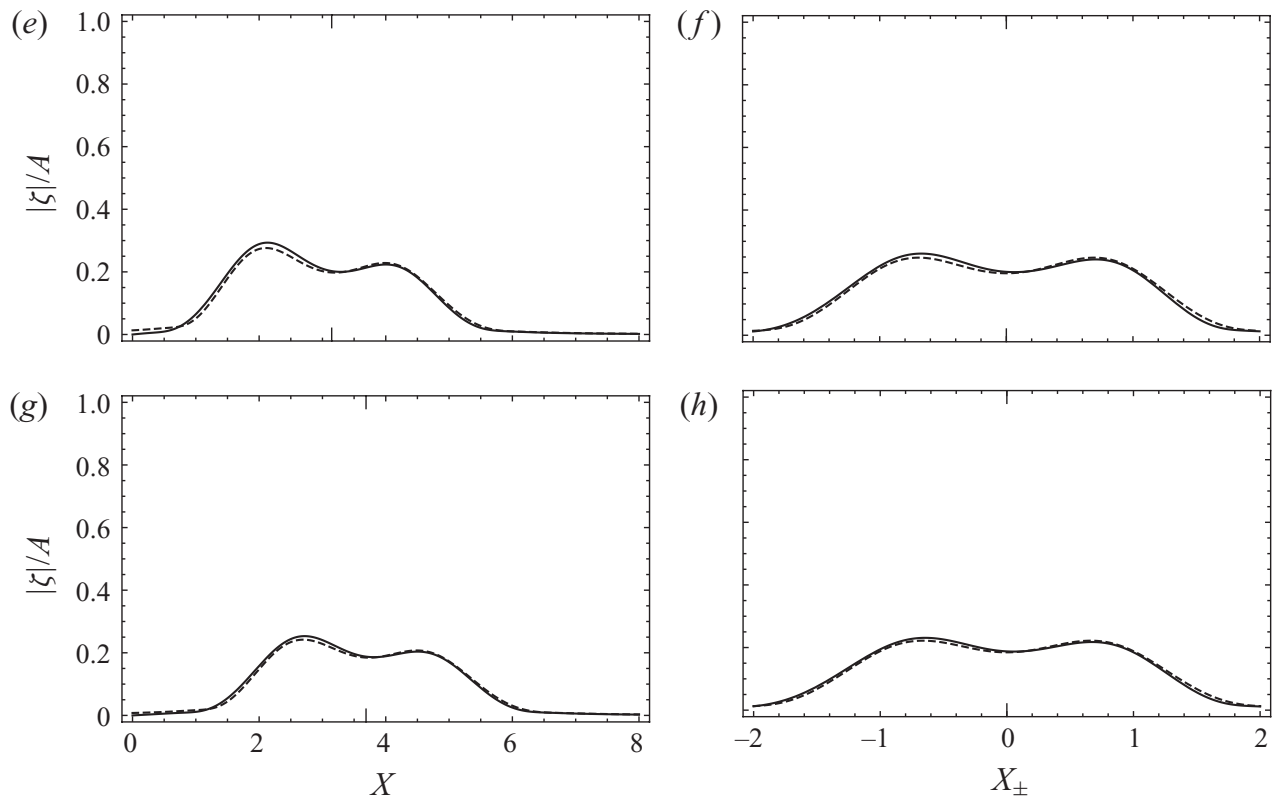

$(h)$

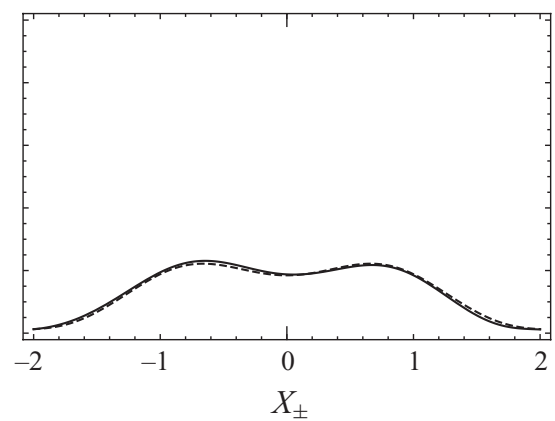

FiguRE 8. Horizontal profiles of the vertical displacement amplitude at $(a)|Z|=1.44$, (c) $|Z|=2.08$, (e) $|Z|=3.48$, (g) $|Z|=4.08$, and transverse profiles at (b) $\left|Z_{ \pm}\right|=1.94$, (d) $\left|Z_{ \pm}\right|=2.80,(f)\left|Z_{ \pm}\right|=4.69$, (h) $\left|Z_{ \pm}\right|=5.50$. The positions of the horizontal profiles and the parameters $\omega / N=0.74, \omega t=41 \pi$ and $R e=1500$ have been chosen to match those for the measurements in figure 22. The positions of the transverse profiles have been chosen such that the two types of profiles intersect at the centreline of the beams, indicated by bigger tick marks. The solid lines represent the exact solution (2.16c) and the dashed lines its far-field approximation (2.17).

where $X_{ \pm}$must be understood as $X_{ \pm}+\mathrm{i} 0$. In practice, as follows from the preceding analysis, unsteady or viscous effects in the laboratory have taken the wave field over long before the far field is reached, so that the adequate representation of the waves there is (2.17) with $\alpha \neq 0$ or $\beta \neq 0$. 


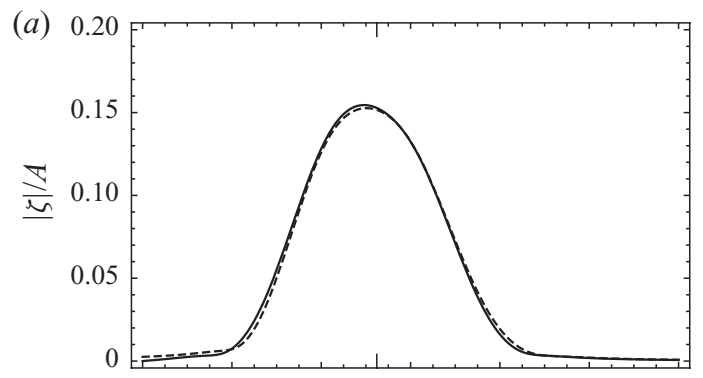

(b)
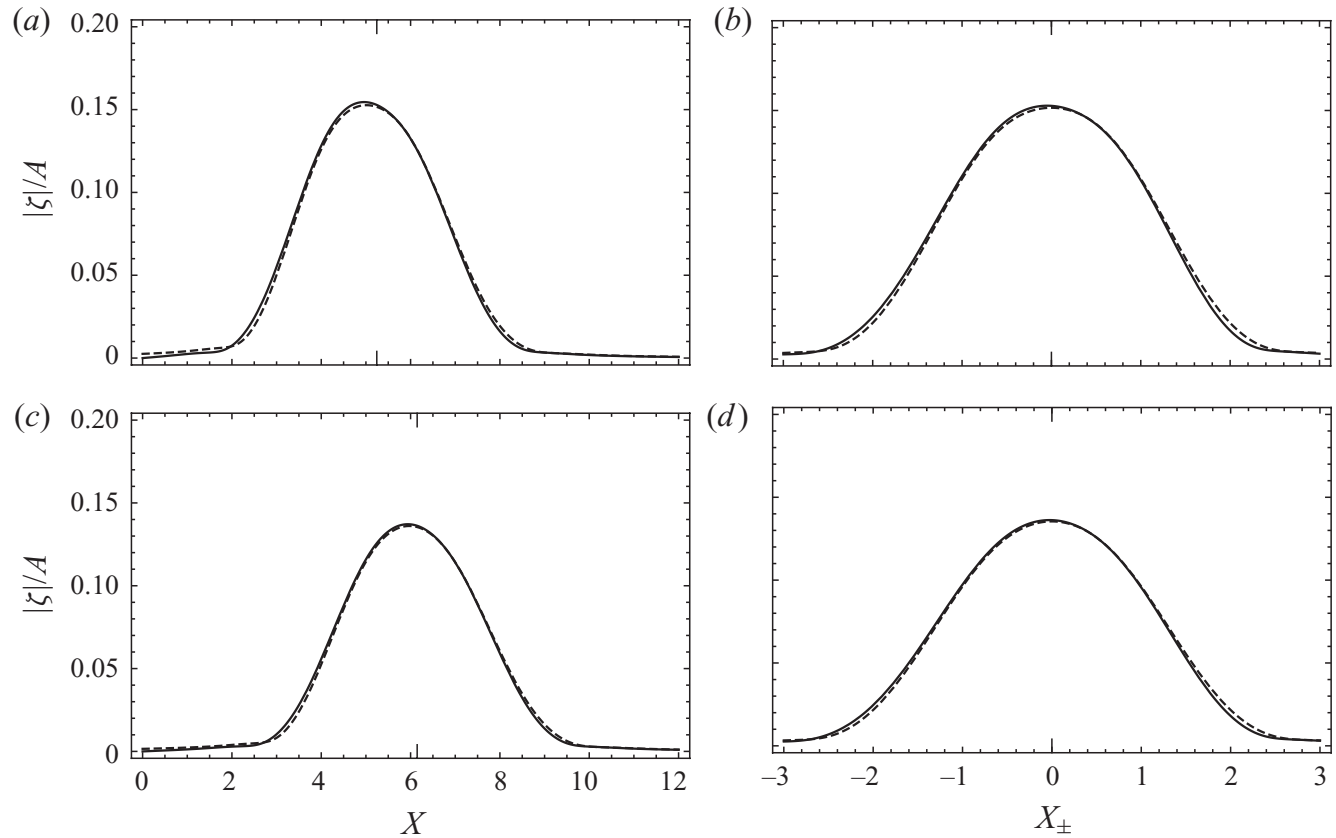

FIGURE 9. Horizontal profiles of the vertical displacement amplitude at $(a)|Z|=5.80$, (c) $|Z|=6.80$, and transverse profiles at $(b)\left|Z_{ \pm}\right|=7.82,(d)\left|Z_{ \pm}\right|=9.17$. The positions of the horizontal profiles and the parameters $\omega / N=0.74, \omega t=41 \pi$ and $R e=520$ have been chosen to match those for the measurements in figure 23 . The mode of representation is the same as in figure 8.

An important consequence of (2.17) is that the transverse wave profile is independent of the angle of oscillation $\eta$ of the sphere, and described by the function

$$
F\left(X_{ \pm}, \sigma_{u}, \sigma_{v} ; n, \mu\right)=\int_{0}^{1 / \sigma_{u}} \exp \left(-\sigma_{v}^{3} K_{ \pm}^{3}\right) K_{ \pm}^{n} \mathrm{~J}_{\mu}\left(K_{ \pm}\right) \exp \left(\mathrm{i} K_{ \pm} X_{ \pm}\right) \mathrm{d} K_{ \pm},
$$

where $n=0$ for the vertical displacement and the longitudinal velocity and 1 for the stability variation, while $\mu=3 / 2$ for the sphere and 1 in the similar functions $I_{e}$ and $I_{o}$ introduced by Hurley \& Keady (1997) for the cylinder. Two non-dimensional parameters have been defined:

$$
\sigma_{u}=\alpha\left|Z_{ \pm}\right|=\frac{\left|Z_{ \pm}\right|}{\omega t \tan \theta}
$$

which quantifies the importance of unsteady effects and may be viewed as the ratio of the transverse scale $\alpha\left|z_{ \pm}\right|$of the waves governed by unsteadiness alone (Makhortykh \& Rybak 1990) to the transverse scale $a$ of the waves governed by the size of the sphere alone (Hendershott 1969; Appleby \& Crighton 1987; Voisin 1991); and

$$
\sigma_{v}=\left(\frac{\beta}{a^{2}}\left|Z_{ \pm}\right|\right)^{1 / 3}=\left(\frac{\left|Z_{ \pm}\right|}{R e \tan \theta}\right)^{1 / 3}
$$

which quantifies the importance of viscous effects and may be viewed as the ratio of the transverse scale $\left(\beta\left|z_{ \pm}\right|\right)^{1 / 3}$ of the waves governed by viscosity alone (Thomas \& Stevenson 1972) to $a$. See Voisin (2003) for the consideration of all three effects in parallel. The relation of $R e$ and $\sigma_{v}$ to the parameters $\lambda$ and $d$ introduced by Hurley \& 
Keady (1997) and used by Sutherland \& Linden (2002), Flynn et al. (2003) and Zhang et al. (2007) is

$$
\lambda=\frac{\beta}{a^{2}}=\frac{1}{R e \tan \theta}, \quad d=\lambda\left|Z_{ \pm}\right|=\sigma_{v}^{3} .
$$

The steady limit $\omega t \rightarrow \infty$ becomes $\sigma_{u} \rightarrow 0$ and the inviscid limit $R e \rightarrow \infty$ becomes $\sigma_{v} \rightarrow 0$.

In a given experiment, namely at given $\omega t$ and $R e$, the variations of $\sigma_{u}$ and $\sigma_{v}$ are not independent. Instead, they are related to the variations of the propagation distance $\left|Z_{ \pm}\right|$according to

$$
\omega t \sigma_{u}=R e \sigma_{v}^{3}=\left|Z_{ \pm}\right| \cot \theta .
$$

Let us imagine temporarily that the far-field solution is valid everywhere. Then $\sigma_{u}$ and $\sigma_{v}$ take their minimal values at the sphere, $1 /(\omega t \tan \theta)$ and $1 /(\operatorname{Re} \tan \theta)^{1 / 3}$ respectively, both small. The wave profile is close to the steady inviscid profile (3.6) and its envelope $|F|$ exhibits two sharp peaks at the edges $\left|X_{ \pm}\right|=1$ of the wave beams; such a profile is called bimodal. As the distance increases, unsteady and viscous effects grow and widen the peaks, which gradually overlap and merge. Ultimately, a single smooth peak at the centre of the beams remains, with small oscillatory tails on either side if $\sigma_{v}$ is small so that the profile is close to unsteady inviscid, and without tail if $\sigma_{u}$ is small so that the profile is close to steady viscous; such a profile is called unimodal.

When near-field effects are taken into account, the wave profile loses its symmetry close to the sphere but the transition from bimodality to unimodality remains, as has been seen in figures 8 and 9 .

The transition is illustrated in figure 10. It was observed first by Ivanov (1989) and Makarov et al. (1990), the latter introducing the terms 'unimodal' and 'bimodal' together with a simple mathematical criterion for the transition: starting from the source and travelling with the wave along the beam, the transition takes place the last time the concavity of the amplitude profile at the centreline changes sign, from a positive to a negative value so that the profile turns from convex into concave. The associated transition distance is

$$
Z_{c}=\max \left\{\left|Z_{ \pm}\right| \mid\left[\frac{\partial^{2}\left|F\left(X_{ \pm}, \sigma_{u}, \sigma_{v} ; n, \mu\right)\right|}{\partial X_{ \pm}^{2}}\right]_{X_{ \pm}=0}=0\right\},
$$

with $\sigma_{u}$ and $\sigma_{v}$ related to $\left|Z_{ \pm}\right|$by (3.11). The transition equation $\left(\partial^{2}|F| / \partial X_{ \pm}^{2}\right)_{X_{ \pm}=0}=0$ has one single solution in the steady case $\sigma_{u}=0$, and infinitely many solutions in the inviscid case $\sigma_{v}=0$ owing to the presence, in the amplitude profile, of secondary peaks associated with transients.

In the $\left(\sigma_{u}, \sigma_{v}\right)$-plane, each experiment, namely each set of values of $\omega t$ and $R e$, travels along a curve (3.11) as $\left|Z_{ \pm}\right|$varies, with (3.12) defining a transition threshold $\left(\sigma_{u_{c}}, \sigma_{v_{c}}\right)$ on the curve. The inviscid limit $R e \rightarrow \infty$ corresponds to the $\sigma_{u}$-axis, and the steady limit $\omega t \rightarrow \infty$ to the $\sigma_{v}$-axis. Considering all possible values of $\omega t$ and $R e$, a transition boundary is obtained in the plane, to separate bimodal and unimodal domains. We introduce the function

$$
I\left(\sigma_{u}, \sigma_{v} ; n, \mu\right)=F\left(0, \sigma_{u}, \sigma_{v} ; n, \mu\right)=\int_{0}^{1 / \sigma_{u}} \exp \left(-\sigma_{v}^{3} K_{ \pm}^{3}\right) K_{ \pm}^{n} \mathrm{~J}_{\mu}\left(K_{ \pm}\right) \mathrm{d} K_{ \pm},
$$

generalizing that introduced by Flynn et al. (2003). The transition equation becomes

$$
I^{2}\left(\sigma_{u_{c}}, \sigma_{v_{c}} ; n+1, \mu\right)=I\left(\sigma_{u_{c}}, \sigma_{v_{c}} ; n, \mu\right) I\left(\sigma_{u_{c}}, \sigma_{v_{c}} ; n+2, \mu\right) .
$$



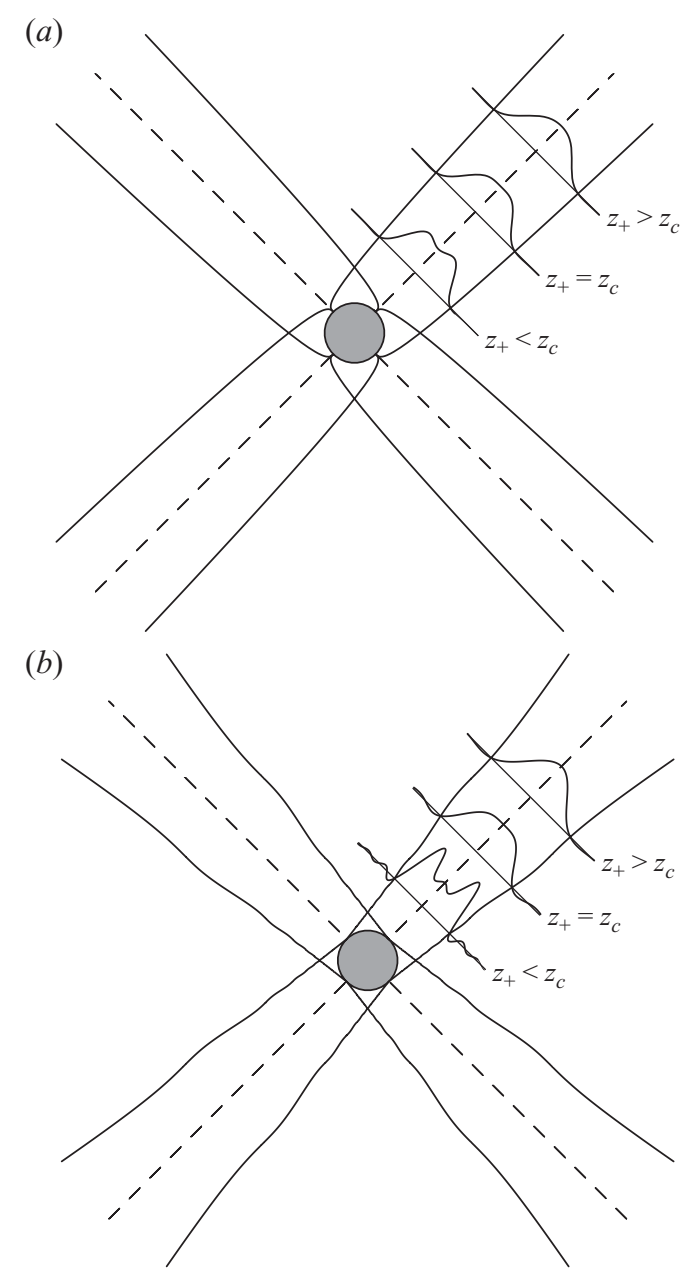

FIGURE 10. Wave beams from a sphere in $(a)$ the steady viscous regime as $\omega t \rightarrow \infty$ with $R e=280$ and $(b)$ the unsteady inviscid regime as $R e \rightarrow \infty$ with $\omega t=20$. For definiteness the beams are drawn for a pulsating sphere and the profiles represent the wave intensity or average rate of energy flux per unit area. Distances are shown to scale with the sphere.

Its solutions and the associated transition boundaries are plotted in figure 11 for $n=0$ corresponding to the vertical displacement and the longitudinal velocity, and $n=1$ corresponding to the stability variation, with $\mu=3 / 2$ corresponding to the sphere. In the inviscid limit, the transition occurs at

$$
\sigma_{u_{c}}=0.18 \quad(n=0), \quad 0.19 \quad(n=1),
$$

and in the steady limit at

$$
\sigma_{v_{c}}=0.22 \quad(n=0), \quad 0.25 \quad(n=1) .
$$

Similar equations, summarized by Chashechkin, Kistovich \& Smirnov (2001), have been obtained for an inclined flat plate oscillating along its plane (Kistovich \& Chashechkin 1999a,b) and a vertical circular cylinder oscillating along its axis (Il'inykh, Smirnov \& Chashechkin 1999). 


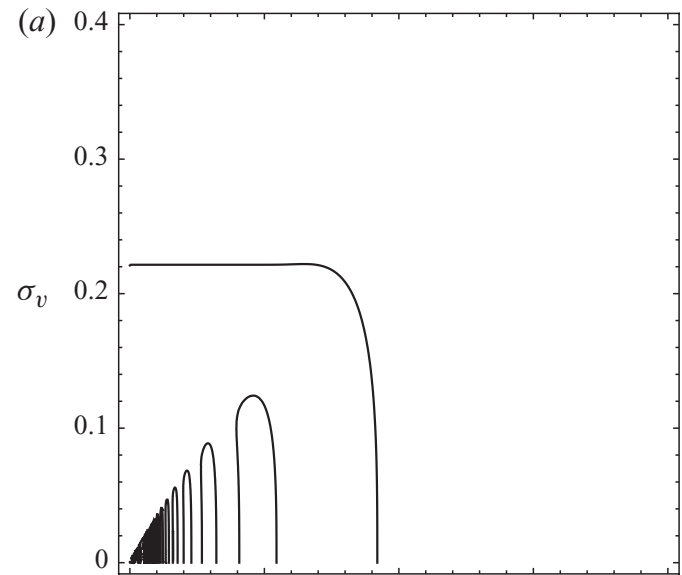

(b)

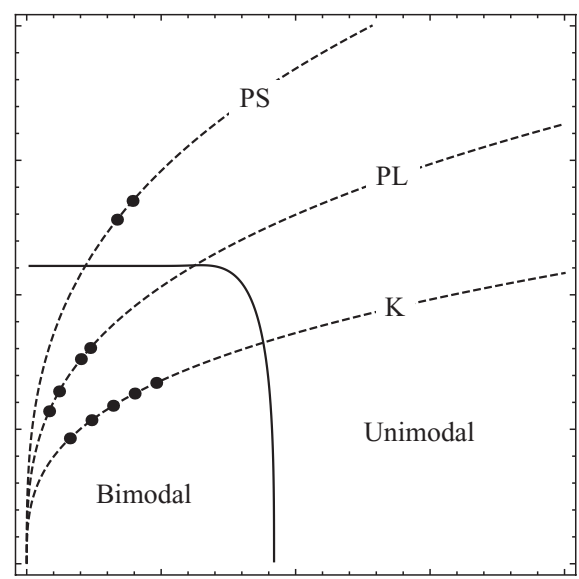

(c)

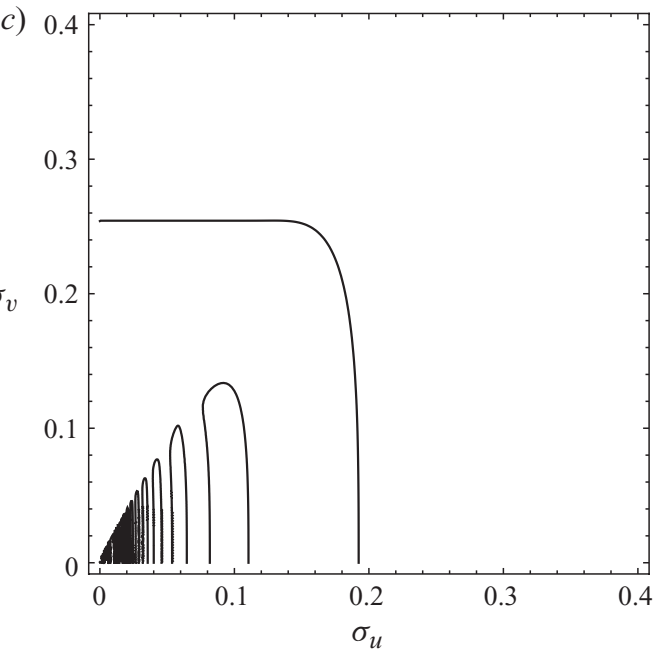

$(d)$

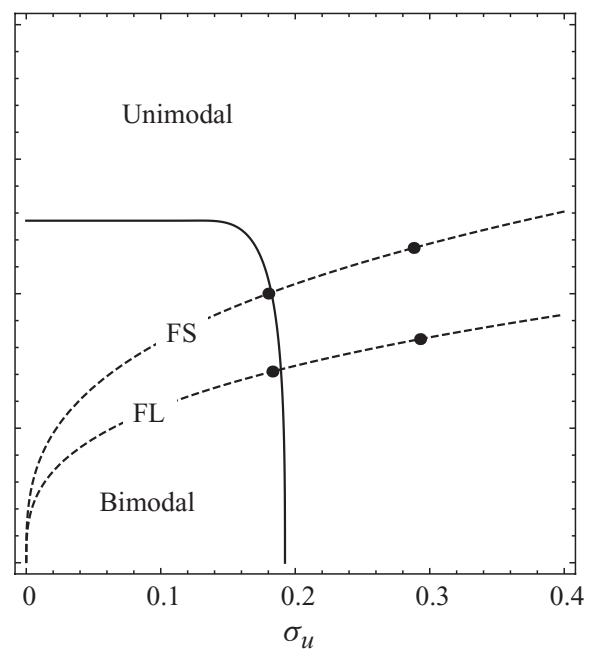

FigURE 11. $(a, c)$ Solutions of the transition equation and $(b, d)$ associated transition diagrams, for $(a, b)$ the vertical displacement and the longitudinal velocity and $(c, d)$ the stability variation. The dashed lines represent the trajectories in the $\left(\sigma_{u}, \sigma_{v}\right)$-plane, as the distance $\left|Z_{ \pm}\right|$from the sphere increases, of: the experiments of Flynn et al. (2003), in which $\omega / N=0.49, \omega t=5 \pi$ and $R e=1000$ for the large sphere (FL) and 350 for the small sphere (FS); the experiments and simulations (K) of King et al. (2009), in which $\omega / N=0.63, \omega t=16 \pi$ and $R e=2000$; and the present experiments, in which $\omega / N=0.74, \omega t=41 \pi$ and $R e=1500$ for the large sphere (PL) and 520 for the small sphere (PS). The dots indicate the distances at which the wave profiles have been measured, namely $\left|Z_{+}\right|=5$ and 8 for Flynn et al. (2003), $\left|Z_{+}\right|=2,3,4,5$ and 6 for King et al. (2009), and $\left|Z_{ \pm}\right|=1.94,2.80,4.69$ and 5.50 for the large sphere and 7.82 and 9.17 for the small sphere in the present experiments.

\section{Relation to the literature}

At this point it is relevant to investigate the relation of the above theoretical results to the literature. We start with the near field, which has only been considered very recently. Table 1 recapitulates all the experimental and numerical studies of oscillating cylinders and spheres known to the authors, together with the values of the relevant non-dimensional parameters, namely the frequency ratio $\omega / N$, the Reynolds-Stokes number $R e$ and the Keulegan-Carpenter number Ke. In all studies but Ermanyuk \& 


\section{Paper}

Görtler (1943)

Mowbray \& Rarity (1967)

Thomas \& Stevenson (1972)

Gordon \& Stevenson (1972)

Peters (1985)

Ivanov (1989)

Makarov et al. (1990)

Sakai (1990)

Xu et al. (1997)

Sutherland et al. (1999)

Sutherland et al. (2000)

Sutherland \& Linden (2002)

Ermanyuk \& Gavrilov (2005)

Zhang et al. (2007)

Mercier et al. (2008)

Ermanyuk \& Gavrilov (2008)

Thomas et al. (2009)

Clark \& Sutherland (2009)

McLaren et al. (1973)

Lin et al. (1994)

Flynn et al. (2003)

Sutherland et al. (2003)

Peacock \& Weidman (2005)

Chashechkin \& Prikhod'ko (2007)

King et al. (2009)

Present paper

Body
Cylinder
Cylinder
Cylinder
Cylinder
Cylinder
Cylinder
Cylinder
Cylinder
Cylinder
Cylinder
Cylinder
Cylinder
Cylinder
Cylinder
Cylinder
Cylinder
Cylinder
Cylinder
Sphere
Sphere
Sphere
Sphere
Sphere
Sphere
Sphere
Sphere

Aspect ratio
1
1
1
1
1
1
1
1
1
1
1
1
1
1
1
1
1
1
1
1
1
1
1
1
1
1
1

\section{$\omega / N$}

Horizontal

Horizontal

Horizontal

Vertical

Vertical

Vertical and inclined

Vertical

Horizontal and vertical

Vertical

Vertical and inclined

Vertical

Vertical

Horizontal

Vertical

Horizontal and vertical

Vertical

Vertical

Vertical

Horizontal

Vertical

Vertical

Vertical

Vertical

Horizontal

Horizontal
0.4-0.8

$0.2-1.0$

0.6

$0.9-1.1$

$0.4-0.9$

$0.2-1.0$

$0.4-0.7$

$0.4-1.0$

$0.2-6.0$

$0.2-0.6$

$0.1-0.7$

$0.1-0.6$

0.7

$0.3,0.4$

0.3

$0.4-2.6$

0.2

$0.7,0.8$

$0.3-1.0$

$0.02-0.8$

$0.3-0.9$

0.5

$0.2-0.9$

0.4-1.

0.6

0.7
Re

$\mathrm{Ke}$

$0.2 \dagger$

60-290† $\quad 0.2-0.5 \dagger$

$30+$

10-20

50-140

180-1200‡

5-350

500-1300

60-3500

120-310

$80-400$

60-330

110

70-1300

120

50-340

$240 \ddagger$

2700,3500

$30-550$

220-1700

350

240-1600

240-960

2000

520,1500
$0.3 \dagger$

$0.2-0.9 \dagger$

$0.02-0.3$

0.03-0.7

$0.3-4.0$

$0.1,0.2$

$0.1-0.2$

$0.1-0.2$

0.6

0.05-0.2

0.1

$0.3-1.2$

0.2

0.7

$0.2-4.8$

$0.1-0.3$

0.3

0.6

$0.2-1.2$

0.05-0.6

$0.2,0.5$

$\dagger$ The amplitude was not specified as peak-to-peak or peak-to-node, and has been assumed peak-to-peak.

$\ddagger$ The kinematic viscosity was not specified, and has been taken as $v=1 \mathrm{~mm}^{2} \mathrm{~s}^{-1}$ typical of saline stratification.

If Owing to background rotation at the angular velocity $\Omega$, the frequency ratio $\omega / N$ has been replaced with $\sqrt{ }\left(\omega^{2}-4 \Omega^{2}\right) /\left(N^{2}-4 \Omega^{2}\right)$.

TABLE 1. Experimental and numerical investigations of oscillating cylinders and spheres in the literature. All cylinders are circular, except for Sutherland \& Linden (2002), who considered an elliptical cylinder with horizontal and vertical principal axes; in this case, the radius is defined as the arithmetic mean of the semi-axes and the aspect ratio as the ratio of the horizontal to vertical axes. 
Gavrilov $(2005,2008)$ the wave field is assumed steady, namely the condition $\omega t \rightarrow \infty$ is assumed to be effectively satisfied.

\subsection{Near field}

The main feature of the near field is the existence of distinct flow structures inside and outside the biconical overlap region circumscribing the sphere. At small amplitude $K e=0.22$, this region is clearly visible in figure $1(a)$ of the experiments of Chashechkin \& Prikhod'ko (2007) for the vertical oscillations of a sphere at $\omega / N=0.73$ and $R e=450$, while their other figures and those of Chashechkin (2007) exhibit the deformation of the region as the amplitude increases, leading ultimately to mixing. Small-scale structures are observed near the poles and equatorial plane, but the visualization method - classical Schlieren - does not allow the determination of the flow field in their neighbourhood.

In two dimensions, an experimental study of the near field has been performed for the horizontal oscillations of a cylinder by Zhang et al. (2007). Their figure 4 exhibits the excellent quantitative agreement between the measurement of the flow field across the diamond-shaped overlap region at $\omega / N=0.40, R e=1300$ and $\mathrm{Ke}=0.05$, and its theoretical prediction by Hurley \& Keady (1997).

\subsection{Far field}

The main feature of the far field is the transition from bimodality to unimodality. Only the steady case $\sigma_{u}=0$ has been considered in the literature. The transition was observed first for the cylinder by Ivanov (1989), who used the term 'far field' for the unimodal region and proposed the estimate $Z_{c} \propto R e \tan \theta$ for the transition distance, and Makarov et al. (1990), who introduced the terms 'bimodal' and 'unimodal' and noticed their connection with the concavity of the amplitude profile at the centreline. The subsequent discussion of Makarov et al. (1990), however, focused instead on the so-called viscous wave scale

$$
\ell_{\nu}=\frac{(g \nu)^{1 / 3}}{N}
$$

such that the waves are everywhere bimodal for $a>\ell_{v}$ while both bimodal and unimodal regions are present for $a<\ell_{\nu}$, with $g$ as the acceleration due to gravity. It turns out that the occurrence of $\ell_{v}$ was a mere artefact of a priori nondimensionalization of the coordinates based on the stratification scale $g / N^{2}$, characterizing the distance over which non-Boussinesq effects become significant, even though these effects play absolutely no role in the dynamics. What can only be said is that, in practice, experiments are designed for non-Boussinesq effects to remain negligible, by ensuring that the tank size is much smaller than $g / N^{2}$. Accordingly, the propagation distance satisfies $\left|Z_{ \pm}\right| \ll g /\left(N^{2} a\right)$ over the whole tank, so that the condition $a>\ell_{v}$ yields $\left|Z_{ \pm}\right| \ll R e$, implying that the transition distance $Z_{c}$ is never reached, while the opposite condition $a<\ell_{v}$ leaves this possibility opened.

Later, Hurley \& Keady (1997), for the cylinder, Kistovich \& Chashechkin $(1999 a, b)$, for an inclined plate oscillating along its plane, and Il'inykh et al. (1999), for a vertical cylinder oscillating along its axis, replaced the transition distance by a transition range, outside which the waves are said to be bimodal for lower $\sigma_{v}$ and unimodal for higher $\sigma_{v}$. Hurley \& Keady (1997) based their definition, $0.1<\sigma_{v}<1.3$ in the present notation, on the longitudinal variations of the amplitude at the centreline: the bimodal condition $\sigma_{v}<0.1$ corresponds to the requirement that the amplitude remains within $1 \%$ of its value at $\sigma_{v}=0$, and the unimodal condition $\sigma_{v}>1.3$ to the requirement that it is well approximated by its leading-order asymptotics as $\sigma_{v} \rightarrow \infty$. 
Kistovich \& Chashechkin $(1999 a, b)$ and Il'inykh et al. (1999) based their definition, $0.12<\sigma_{v}<0.33$ for the plate in the present notation, on the concavity of the amplitude profile at the centreline: for their specific forcing mechanisms, the equation of zero concavity $\left(\partial^{2}|F| / \partial X_{+}^{2}\right)_{X_{+}=0}=0$ has three separate roots, and the transition range is defined as the interval between the first and third roots.

Flynn et al. (2003) considered the two types of transition criteria in parallel, for the cylinder and the sphere. First, definition (3.12) of the transition distance was used, based on the concavity of the amplitude profile at the centreline. Values consistent with (3.16) were obtained for the sphere, and the values obtained for the cylinder have been verified experimentally by Zhang et al. (2007). Second, an alternative definition was proposed, based on the longitudinal variations of the amplitude at the centreline and placing the transition at the distance where this amplitude, normalized by its dependence as $\left|Z_{ \pm}\right|^{-\epsilon}$ at $\sigma_{v}=0$, with $\epsilon=1 / 2$ for the sphere and 0 for the cylinder, reaches a maximum before decreasing ultimately according to its leadingorder asymptotics as $\sigma_{v} \rightarrow \infty$. Mathematically this is expressed as

$$
Z_{c}^{\star}=\max \left\{\left|Z_{ \pm}\right| \mid\left[\frac{\partial\left|F\left(X_{ \pm}, \sigma_{u}, \sigma_{v} ; n, \mu\right)\right|}{\partial\left|Z_{ \pm}\right|}\right]_{X_{ \pm}=0}=0\right\} .
$$

In terms of $I\left(\sigma_{u}, \sigma_{v} ; n, \mu\right)$, the transition equation $\left(\partial|F| / \partial\left|Z_{ \pm}\right|\right)_{X_{ \pm}=0}=0$ becomes

$$
I\left(\sigma_{u_{c}}^{\star}, \sigma_{v_{c}}^{\star} ; n+3, \mu\right)+\frac{\mathrm{J}_{\mu}\left(1 / \sigma_{u_{c}}^{\star}\right)}{\left(\sigma_{u_{c}}^{\star}\right)^{n+1}\left(\sigma_{v_{c}}^{\star}\right)^{3}} \exp \left[-\left(\frac{\sigma_{v_{c}}^{\star}}{\sigma_{u_{c}}^{\star}}\right)^{3}\right]=0,
$$

and simplifies to $I\left(0, \sigma_{v_{c}}^{\star} ; n+3, \mu\right)=0$ in the steady case $\sigma_{u}=0$ considered by Flynn et al. (2003). However, this equation does not always admit of a solution, and when it does this solution does not differ significantly from that of (3.14).

\subsection{Wave profiles}

The only quantitative measurements of the waves from a sphere in the literature have been performed by Flynn et al. (2003), Sutherland et al. (2003) and King et al. (2009).

Flynn et al. (2003) and Sutherland et al. (2003) have considered vertical oscillations experimentally. We focus on the former. Two spheres were used, one small with $R e=350$ and $K e=0.15,0.19$ and 0.26 , the other large with $R e=1000$ and $K e=0.10$, 0.18 and 0.27 , both oscillating at $\omega / N=0.49$. The initial phase was $\Theta=\pi$, such that the oscillations started from their centre downwards. For each sphere, two transverse profiles were measured at the distances $Z_{+}=5$ and 8 , and two longitudinal profiles along the centre $X_{+}=0$ and inviscid edge $X_{+}=-1$ of the upper wave beam. The plotted quantity was the amplitude $\left|\Delta N^{2}\right|$ of the stability variation.

The axisymmetric geometry prevented absorbers from being positioned at the tank walls, thus preventing all reflections while leaving the walls available for visualization. As a consequence, the measurements had to be performed as early as possible, before significant reflection could take place. Two periods after the start-up, the wave field appeared to be steady. Accordingly, the measurements were performed during the third period $2<t / T<3$, with $T=2 \pi / \omega$ as the period of oscillation. The plots were compared with theoretical predictions obtained by applying to the sphere the same method that Hurley (1997), Hurley \& Keady (1997) and Hurley \& Hood (2001) had applied to the cylinder; namely, writing the steady inviscid solution of Hendershott (1969), Sarma \& Krishna (1972), Appleby \& Crighton (1987) and Voisin (1991) in a form allowing the inclusion of the viscous attenuation factor derived by Lighthtill $(1978, \S \S 4.7$ and 4.10). 


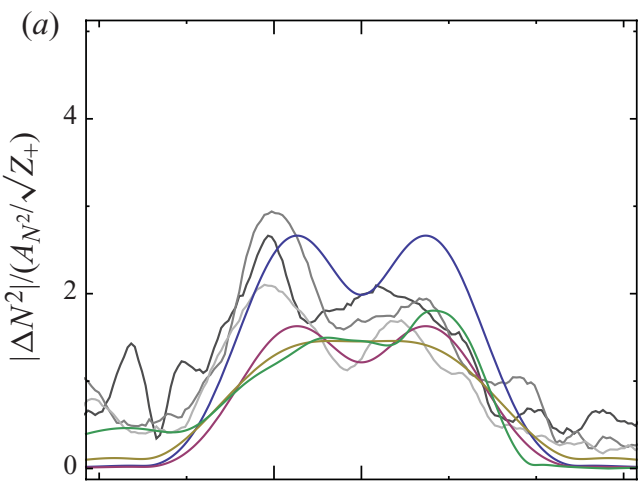

(b)
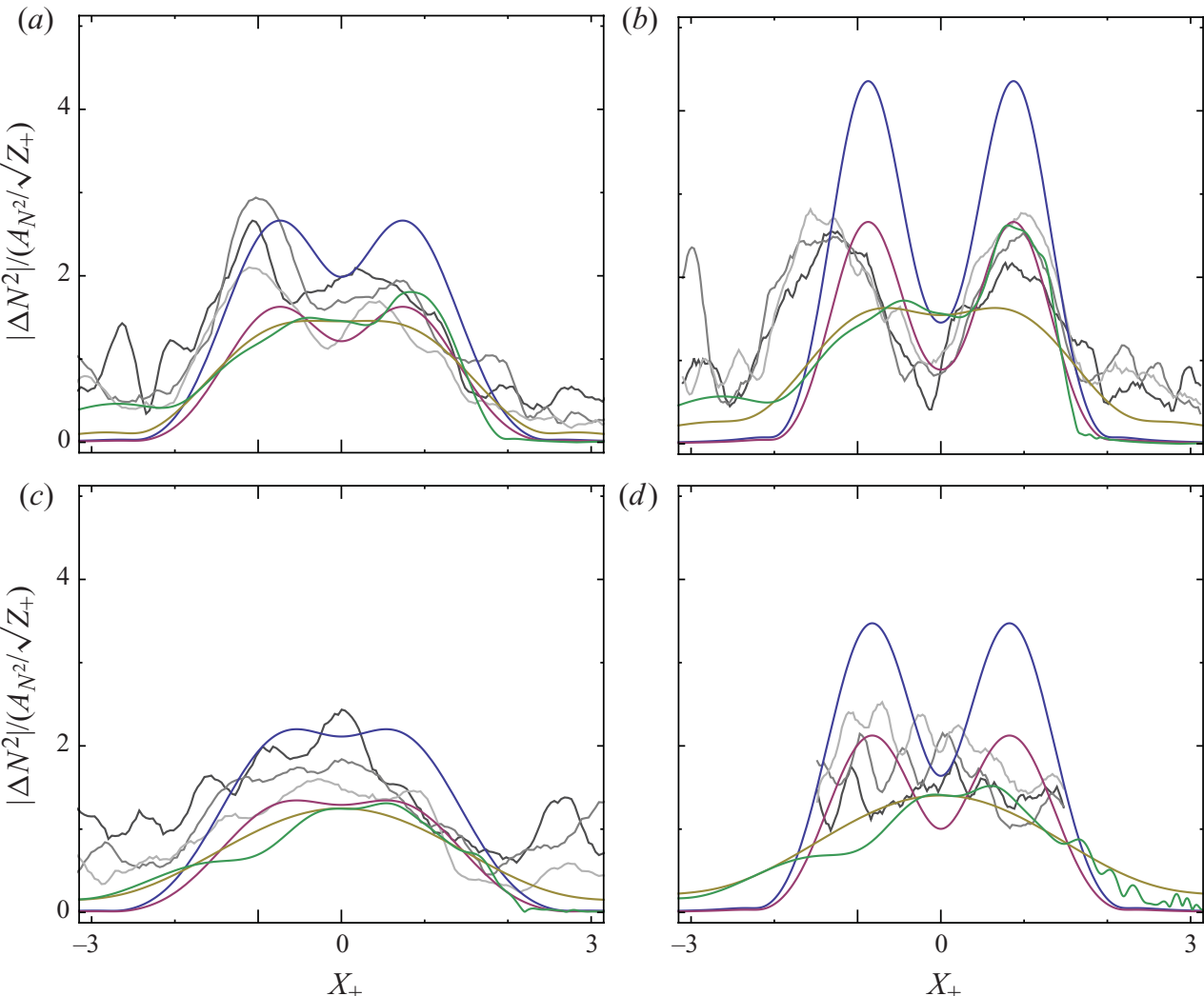

$(d)$

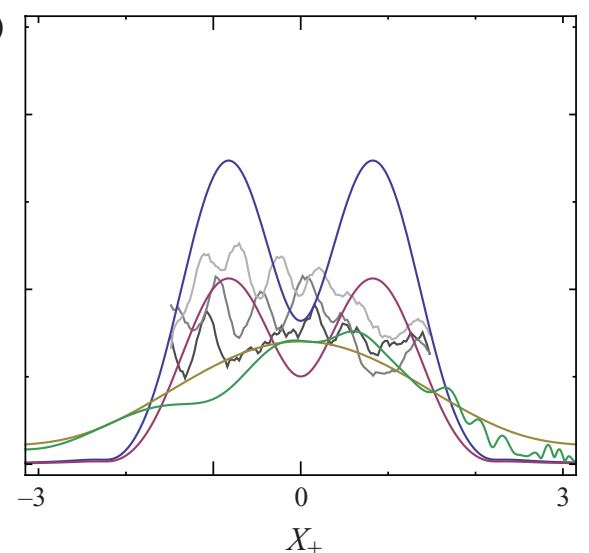

FIGURE 12. Transverse profiles of the stability variation amplitude in the experiments of Flynn et al. (2003), at $(a, b) Z_{+}=5$ and $(c, d) Z_{+}=8$ for $(a, c)$ the small sphere and $(b, d)$ the large sphere. The grey lines represent the experimental data at $K e=0.15$ (black), 0.19 (grey) and 0.26 (light grey) for the small sphere, and $K e=0.10$ (black), 0.18 (grey) and 0.27 (light grey) for the large sphere. The colour lines represent the steady far-field theory with no effect of stratification on added mass (blue), as used by Flynn et al. (2003), and its modification by the cumulative addition of the effects of stratification on added mass (purple), of unsteady effects at time $t / T=2.5$ (dark green), and of near-field effects (light green). The amplitude has been normalized by $A_{N^{2}}=\left(N^{2} / 2\right)(A / a) \sin \theta \cos \theta$, and the bigger tick marks indicate the positions of the longitudinal profiles from figure 13 .

The comparison is reproduced in figures 12 and 13, corresponding to figures 8-11 of Flynn et al. (2003). The predicted transverse profiles are all bimodal and symmetrical, whereas the measured profiles are bimodal at $Z_{+}=5$, symmetrical for the large sphere and asymmetrical for the small sphere, and unimodal at $Z_{+}=8$, with a high noise level; in addition, the amplitude is overpredicted for the large sphere by almost a factor 2. The longitudinal profile is also overpredicted for the large sphere at $X_{+}=-1$, and exhibits at $X_{+}=0$ an unexpected re-increase with distance.

In order to propose explanations for these discrepancies, we look more closely at each approximation involved. The comparison of (2.15) of Flynn et al. (2003) with the present (2.17) reveals that in addition to explicit far-field and steady approximations, an implicit approximation $B(\cos \theta)=1 / 3$ has been introduced inadvertently by Flynn et al. (2003), equivalent to the approximation (2.13) of Gorodtsov \& Teodorovich (1982, 1986). Hurley (1997) and Hurley \& Keady (1997) have shown that this 


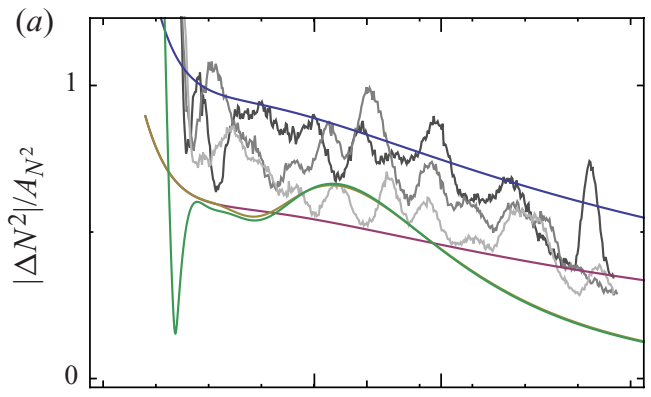

(b)
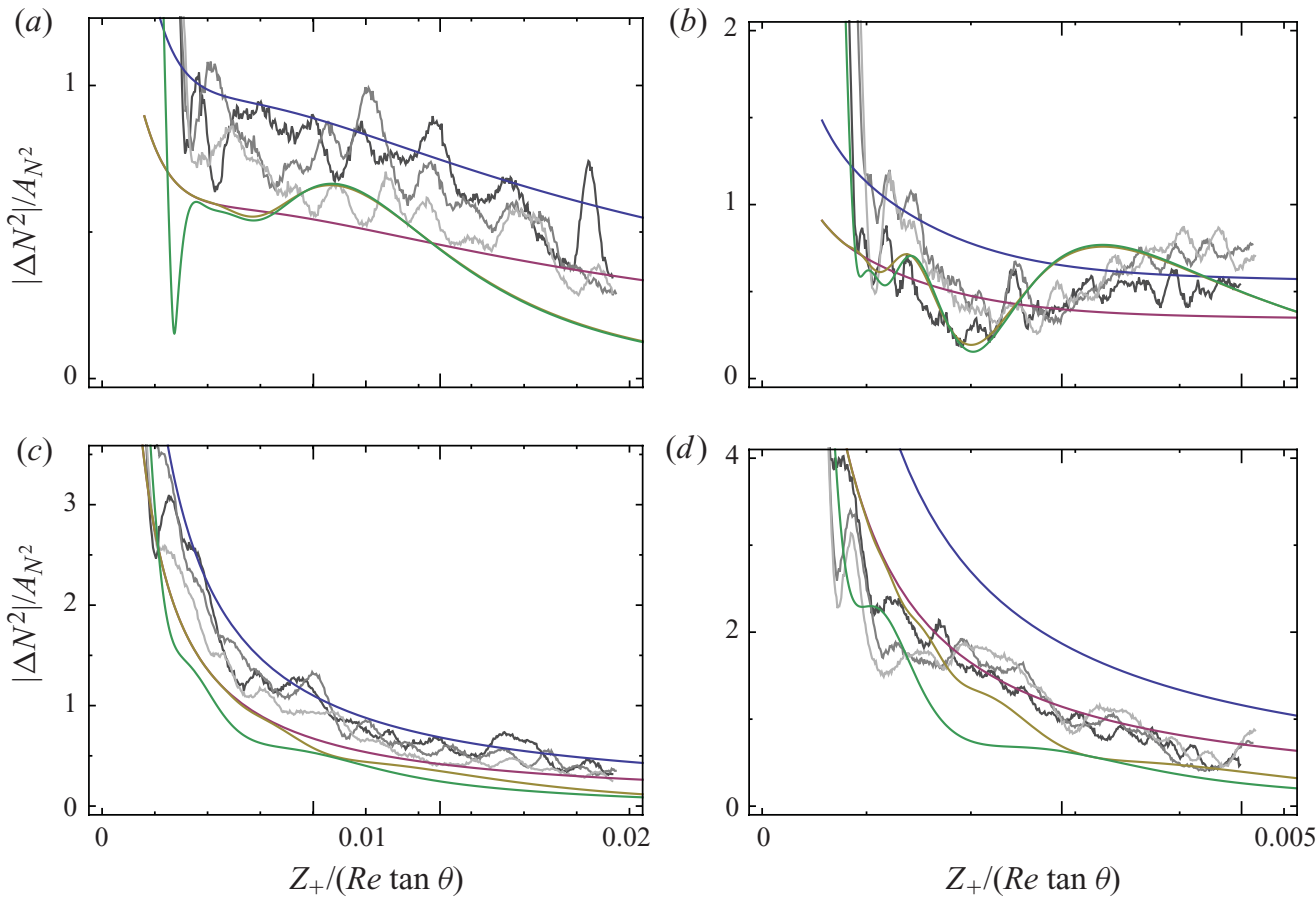

FIGURE 13. Longitudinal profiles of the stability variation amplitude in the experiments of Flynn et al. (2003), at $(a, b) X_{+}=0$ and $(c, d) X_{+}=-1$ for $(a, c)$ the small sphere and $(b, d)$ the large sphere. The mode of representation is the same as in figure 12, and the bigger tick marks indicate the positions of the transverse profiles from figure 12 .

approximation is erroneous for the cylinder; we see here that it is also erroneous for the sphere. Indeed, reinstating the correct form $(2.15)$ of $B(\cos \theta)$ in (2.17) yields satisfactory prediction of the peak amplitude for the large sphere in figures 12 and 13.

The steady approximation has been considered at the foci in figure $5(a)$, concluding that the waves are effectively steady over the measurement period. Further away, the steady state takes longer to reach: in figure 14 it is seen to start at, say, $t / T=3$ for the small sphere and 5 for the large sphere at $Z_{+}=5$, and at, say, $t / T=5$ for the small sphere and 7 for the large sphere at $Z_{+}=8$. Accordingly, none of the transverse profiles is steady. Reinstating unsteady effects in (2.17), we see that the unimodal character of the transverse profiles at $Z_{+}=8$ in figure $12(c, d)$ is elucidated, consistent with the transition diagram in figure $11(d)$, and that the re-increase of the amplitude with distance at $X_{+}=0$ in figure $13(b)$ for the large sphere is also elucidated.

Detailed experimental studies of the temporal evolution of the waves from a cylinder have been performed by Ermanyuk \& Gavrilov (2005), who concluded that for vertical oscillations at $R e=110$ and $\omega / N=0.70$ the steady state is reached at, say, $t / T=5$ for $Z_{+}=5$ and $t / T=6$ for $Z_{+}=8$, and Ermanyuk \& Gavrilov (2008), who concluded that for horizontal oscillations at $R e=100$ and $\omega / N=0.76$ the steady state is reached at, say, $t / T=7$ for $Z_{+}=12$. In both cases, a duration of 10 periods after the start-up was considered a safe estimate, ensuring that the waves are steady over typical measurement distances. Similar durations were observed for the sphere by Chashechkin \& Prikhod'ko (2007), who counted 10 periods for vertical oscillations 

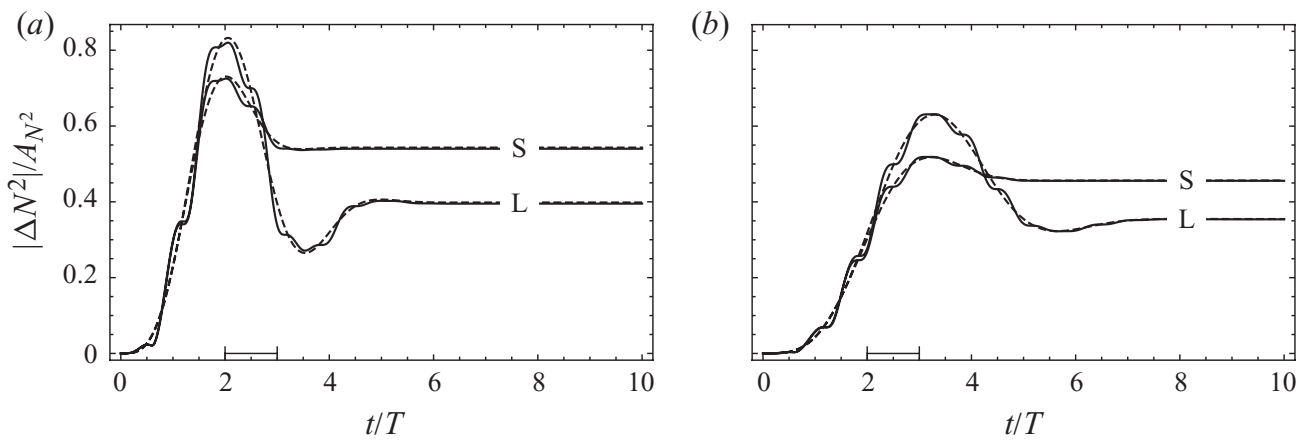

FIGURE 14. Temporal evolution of the stability variation amplitude at the centreline, at (a) $Z_{+}=5$ and (b) $Z_{+}=8$ for the large sphere (L) and the small sphere (S) in the experiments of Flynn et al. (2003). The solid lines represent the exact prediction (2.16) and the dashed lines its far-field approximation (2.17). The horizontal segments indicate the measurement interval $2<t / T<3$.
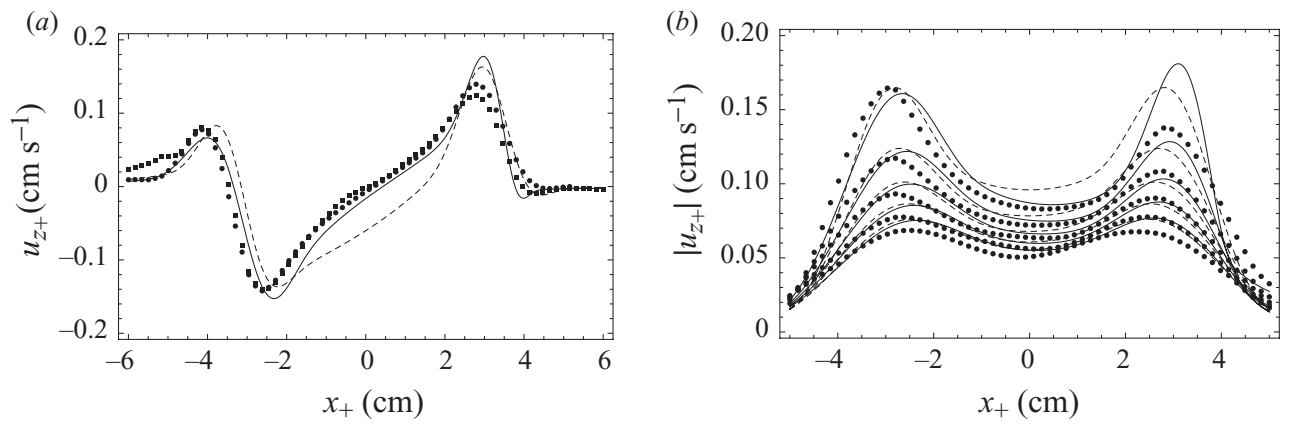

FIGURE 15. Transverse profiles of the longitudinal velocity in the experiments and simulations of King et al. (2009). The profiles are measured in the seventh-tenth periods $6<t / T<10$, for $(a)$ the instantaneous profile at $Z_{+}=2$ and phase $11 \pi / 10$ after the start of a period, and (b) the amplitude profiles at $Z_{+}=2,3,4,5$ and 6 (from top to bottom). The dots represent the experimental data (ם) and the numerical data $(\bullet)$, while the solid lines represent the exact prediction (2.16) and the dashed lines its far-field approximation (2.17).

at $R e=450$ and $\omega / N=0.73$, and King et al. (2009), who counted six periods for horizontal oscillations at $R e=2000$ and $\omega / N=0.63$.

The far-field approximation is relaxed by replacing (2.17) by (2.16). The variations of the amplitude at small distances at $X_{+}=0$ in figure 13(b) for the large sphere are seen to be attributable to near-field effects, but the asymmetry of the transverse profile at $Z_{+}=5$ in figure $12(a)$ for the small sphere remains unexplained. As a rule, the noise level in the measurements is too large for definitive conclusions to be drawn. This provided the motivation for the present experiments.

Before moving on to them, we look briefly at the measurements of King et al. (2009), who considered horizontal oscillations experimentally and numerically. A single sphere was used in the experiments, with $\omega / N=0.63, \operatorname{Re}=2000$ and $\mathrm{Ke}=0.09$. The initial phase was $\Theta=\pi / 2$, such that the oscillations started from their right extremity to the left. Transverse profiles of the longitudinal velocity $u_{z_{+}}$were measured, instantaneous at $Z_{+}=2$ and of the amplitude at $Z_{+}=2,3,4,5$ and 6 , yielding figures 3(c) and 6 of King et al. (2009), respectively. A comparison with the present theory is proposed in figure 15. Quantitative agreement is excellent. The profiles are 

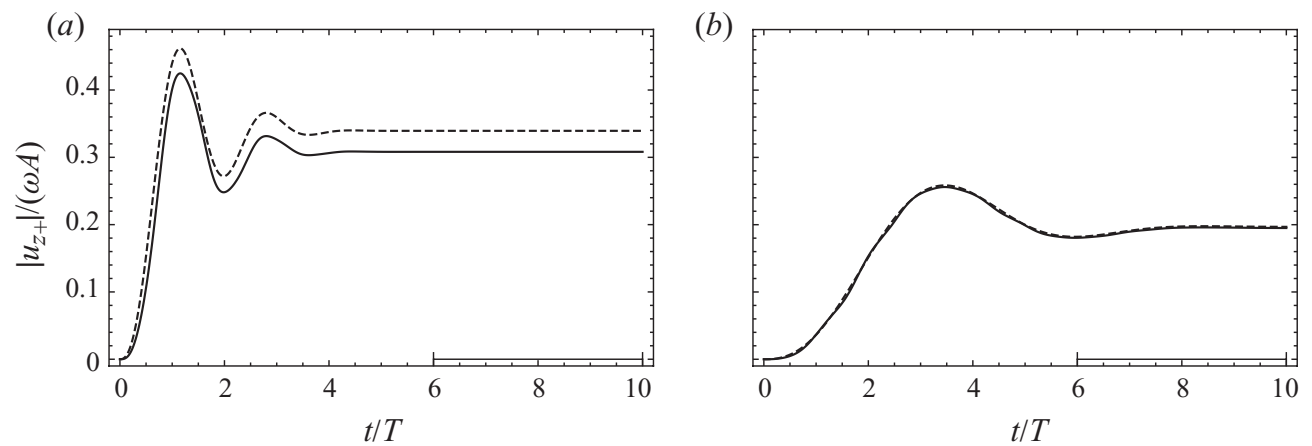

FIGURE 16. Temporal evolution of the longitudinal velocity amplitude at the centreline, at (a) $Z_{+}=2$ and (b) $Z_{+}=6$ in the experiments and simulations of King et al. (2009). The solid lines represent the exact prediction (2.16) and the dashed lines its far-field approximation (2.17). The horizontal segments indicate the measurement interval $6<t / T<10$.

bimodal, as expected from figure 11(b). Near-field effects are seen to be responsible for the shape of the profile near the centre of the beams, but fail to account for the observed asymmetry of the peaks.

The measurements were performed in the seventh-tenth periods, that is, for $6<t / T<10$, after determining numerically that, by the seventh period, the waves were within $1.3 \%$ of their asymptotic values at points in the wave field. The prediction of the temporal evolution of the longitudinal velocity at $Z_{+}=2$ and 6 in figure 16 supports this view.

\section{Experimental procedure}

\subsection{Set-up}

The present experiments were conducted in a Plexiglas square tank of length $97 \mathrm{~cm}$, width $97 \mathrm{~cm}$ and height $50 \mathrm{~cm}$, represented in figure 17. The tank was filled to a depth of $47 \mathrm{~cm}$ with a linearly stratified fluid using the 'double-bucket' method (Fortuin 1960; Oster \& Yamamoto 1963; Oster 1965). Salt was used as stratifying agent and tap water as working fluid. The linearity of the stratification was checked by taking samples at different heights in the fluid and measuring their density with a highprecision Anton Paar density meter. The buoyancy frequency was kept constant for the different experiments at $N=1.22 \mathrm{~s}^{-1}$, and the kinematic viscosity $v=1.2 \mathrm{~mm}^{2} \mathrm{~s}^{-1}$ was also constant.

The waves were generated by a Plexiglas sphere attached to a pendulum of length $l=1.3 \mathrm{~m}$. The oscillations of the pendulum were driven by a wheel of $7 \mathrm{~cm}$ in diameter, placed at mid-height and rotating around an eccentric axis. The pendulum was pushed against the wheel by a counterweight mounted near the pivot. The amplitude of the oscillations was varied by changing the vertical position of the motor. Their frequency was kept constant at $\omega=0.905 \mathrm{~s}^{-1}$, so that their period was $T=6.94 \mathrm{~s}$ and the frequency ratio was $\omega / N=0.74$. The amplitude $A$ of the oscillations of the sphere was kept small compared to the length of the pendulum $(A / l<0.016)$, such that the motion of the sphere was in good approximation rectilinear and horizontal. The initial phase was $\Theta=\pi$, such that the oscillations started from their centre to the left. Two spheres were used, one small of radius $a=1.85 \mathrm{~cm}$ and the Reynolds-Stokes number $R e=520$, and the other large of radius $a=3.125 \mathrm{~cm}$ and the Reynolds-Stokes number $R e=1500$. 
(a)

Pendulum

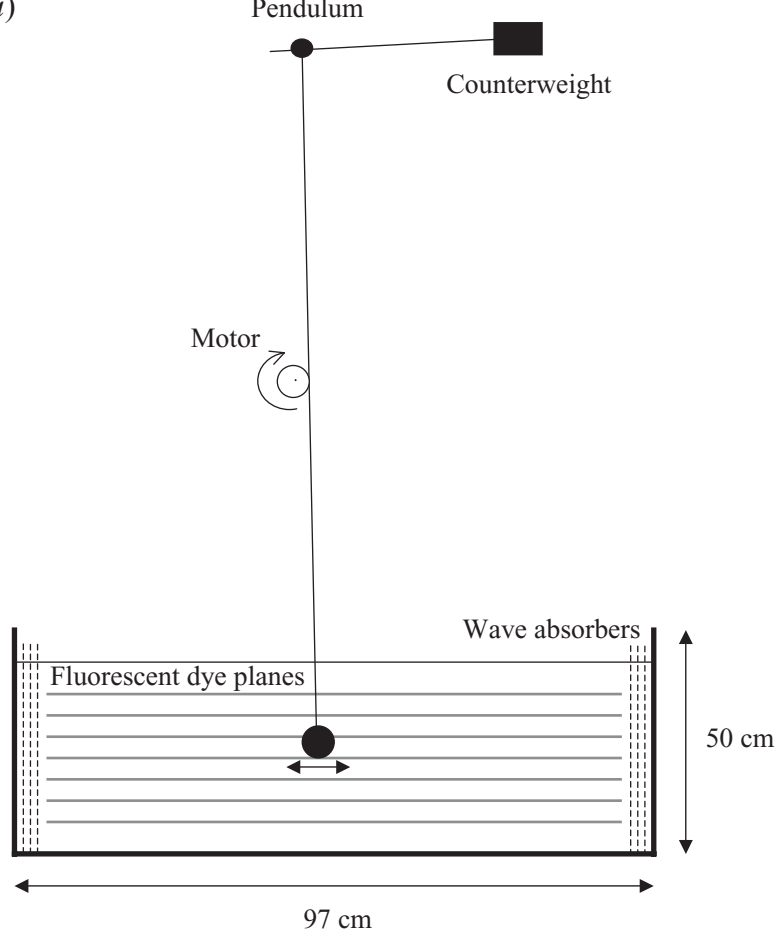

(b)

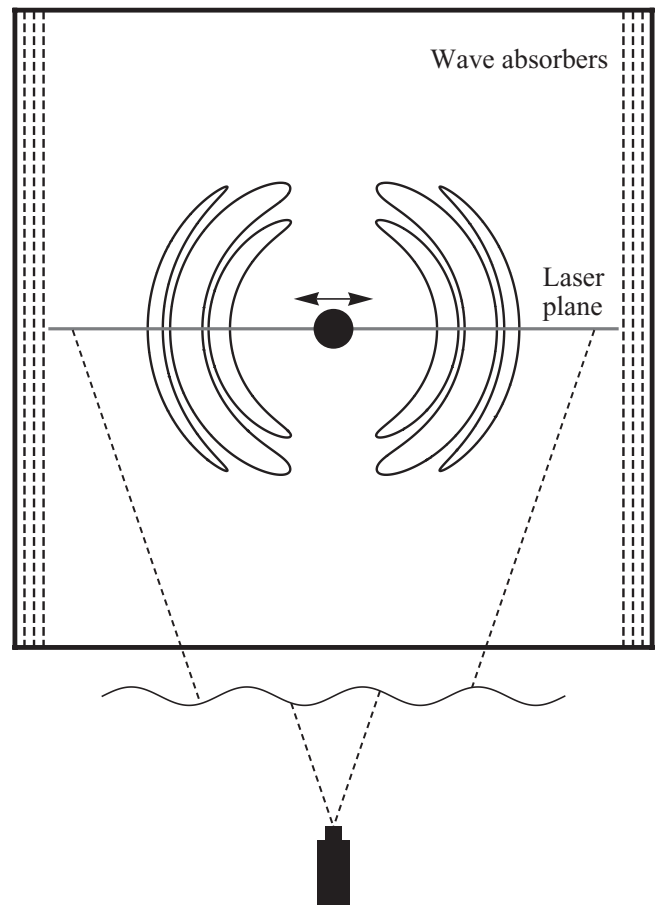

FIGURE 17. Experimental set-up in $(a)$ side view and $(b)$ top view. In the latter, the theoretical isolines $|\zeta| / A=0.04$ are plotted for the large sphere at $Z=-8$, illustrating the position of the waves just before reflection at the bottom of the tank. 
The waves were recorded from the side with a 12 bit DALSA camera with CCD matrix of $1024 \times 1024$ pixels. The field of view was $37 \times 37 \mathrm{~cm}^{2}$, with the sphere at its centre. The analysed area was $0 \leqslant x \leqslant 18 \mathrm{~cm}$ by $-16 \leqslant z \leqslant 10 \mathrm{~cm}$ in a Cartesian coordinate system with origin at the centre of oscillation. Since the camera was located at a distance of $1.5 \mathrm{~m}$ from the tank, parallax errors were negligibly small.

Reflection of the waves at the sidewalls was suppressed by taking advantage of the radiation pattern of the waves, following from the factor $\cos \varphi$ in (2.16)-(2.17), such that, in the horizontal plane, the waves propagate in the direction of oscillation and are attenuated in the perpendicular direction, as illustrated in figure $17(b)$. This pattern has since been verified experimentally and numerically by King et al. (2009). The two walls perpendicular to the direction of oscillation were equipped with absorbers and the other two walls remained available for visualization. The absorbers were made of three layers of a diagonally placed plastic grid of mesh size $2 \times 2 \mathrm{~cm}^{2}$ separated from one another by about $2 \mathrm{~cm}$. Their presence reduced the effective width of the tank by about $10 \mathrm{~cm}$ on each side. The wave energy being proportional to the wave amplitude squared, the range of azimuthal angles $\varphi$ that the absorbers covered $\left( \pm 52^{\circ}\right)$ represented $88 \%$ of the total radiated energy, implying that this energy was in good approximation dissipated by the absorbers. In practice, no wave reflection was observed at the walls and less than $2 \%$ of the total wave energy was radiated towards the camera.

With wall reflection suppressed, it was possible to wait long enough for the steady state to be reached. Under typical experimental conditions 10 periods were sufficient, consistent with the discussion in $\S 4.3$. In order to avoid any ambiguity, the data were recorded after 20 periods, that is, in the interval $20<t / T<21$.

\subsection{Fluo-line technique}

The waves were measured using the same method introduced by Hopfinger et al. (1991) and Bonnier et al. (1998) for the visualization of lee waves and wakes, and Flór et al. $(2002,2004)$ for the measurement of isopycnal displacements. Prior to the experiment a rake of horizontally spanned thin cotton wires, soaked in a fluorescein solution of high concentration and dried, was carefully displaced through the fluid creating a set of equidistant dye planes about $2 \mathrm{~cm}$ apart. The sphere was introduced carefully into the fluid and the whole system left in rest for about $10 \mathrm{~min}$, so that all perturbations could decay. During that time molecular diffusion, which was high during the first two hours because of high gradients and small afterwards, caused a Gaussian distribution of dye across each plane. Then the planes were illuminated by a vertical laser sheet about $2 \mathrm{~mm}$ thick through the centre of the sphere along its direction of oscillation, and the experiment started. To ensure sufficient contrast, a black screen serving as background was fixed to the back wall of the tank. A typical experimental image is reproduced in figure 18 .

The Gaussian dye distribution allows for the accurate measurement of the position of each plane, with sub-pixel resolution. Along a vertical line the light intensity $I(z)$ varies as a sequence of Gaussian distributions superposed with random noise. To determine the centre of each distribution, we calculate the cross-correlation of the experimental signal with a standard Gaussian function

$$
G\left(z-z^{\prime}, s\right)=\frac{1}{(2 \pi)^{1 / 2} s} \exp \left[-\frac{\left(z-z^{\prime}\right)^{2}}{2 s^{2}}\right]
$$




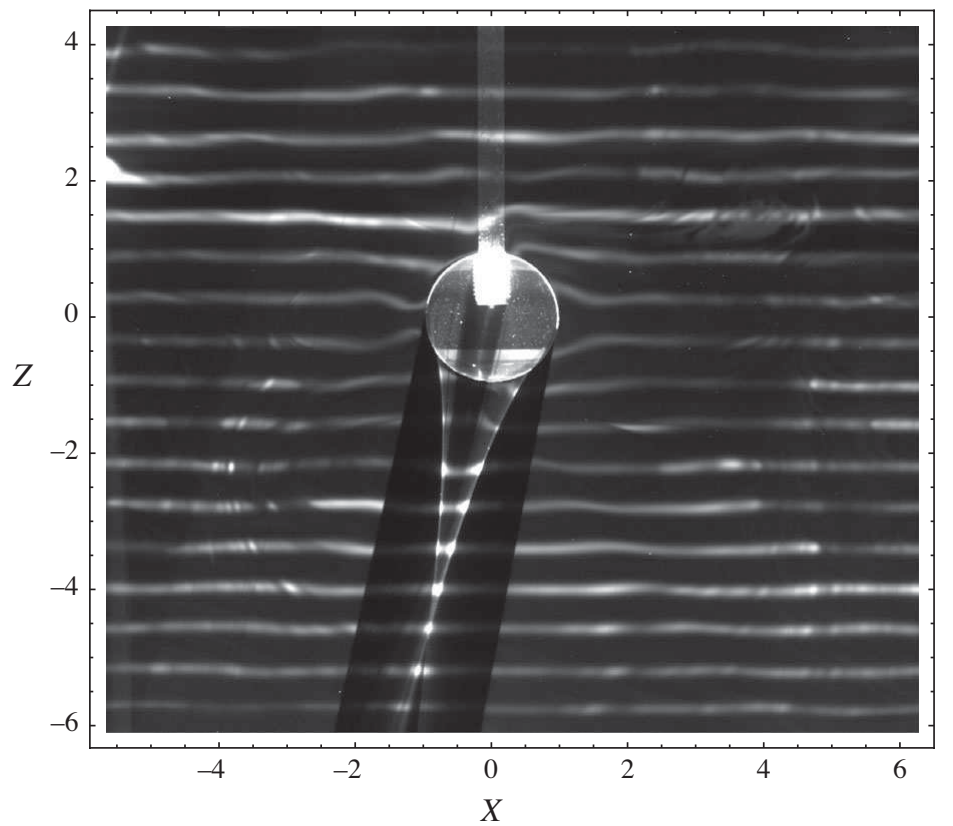

FiguRE 18. Observed dye pattern for the large sphere at $K e=0.18$. The image is taken 20 periods and $3.38 \mathrm{~s}$ after the start-up and is 1024 pixels wide by 889 pixels high, the sphere having a radius of 86 pixels.

of fixed width $2 s$ and moving centre at $z=z^{\prime}$. The cross-correlation function,

$$
C(z)=\int I\left(z^{\prime}\right) G\left(z^{\prime}-z, s\right) \mathrm{d} z^{\prime},
$$

has maxima at the positions of the Gaussian maxima in the signal. For computational efficiency it is written in the form

$$
C(z)=\int G\left(z^{\prime \prime}, s\right) I\left(z^{\prime \prime}+z\right) \mathrm{d} z^{\prime \prime},
$$

namely the Gaussian function is calculated once with fixed centre and the experimental signal is displaced with respect to it. This signal being measured in discrete coordinates with an increment of 1 pixel, the Gaussian function $G_{j}$ is also calculated in discrete coordinates, on $N=4 s+1$ pixels in order to make sure that its tails are sufficiently close to zero. The cross-correlation function becomes

$$
C_{n}=\sum_{j=0}^{N-1} G_{j} I_{j+n} .
$$

Its values $C_{n}$ are used as nodes for interpolation by cubic splines, and the maxima of the interpolated curve are determined with an accuracy of 0.1 pixel, corresponding to the estimated noise level in the experiments (based on dye plane thickness and picture quality). For very thin dye planes, the accuracy can be as high as 0.03 pixel. The cross-correlation procedure is illustrated in figure 19.

The width $2 s$ of the theoretical Gaussian function has been chosen to coincide with the typical width of the experimental Gaussian distributions, determined by a leastsquares fit to the data. This choice represents a good compromise between spatial 


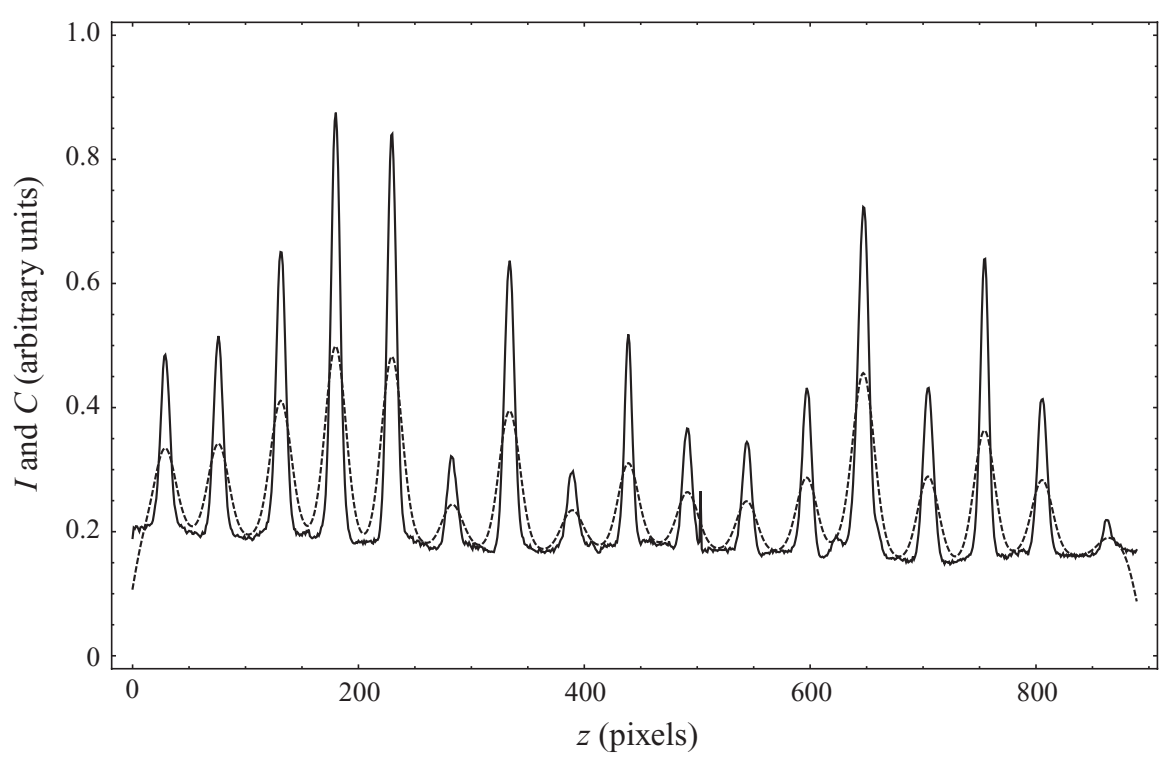

FIGURE 19. Profiles of light intensity $I$ (solid line) and cross-correlation $C$ with a moving Gaussian function (dashed line) along a vertical line of pixels from figure 18.

resolution (which improves at lower $s$ ) and noise filtering (which improves at higher $s)$. In the present experiments the typical width was about 16 pixels, yielding $s=8$. In addition, for each vertical line of pixels the intensity profile $I_{j}$ was averaged over six neighbouring lines, to reduce the duration of the calculations together with the experimental noise arising from tiny defects (scratches and impurities) at the sidewalls of the tank. The average did not affect the horizontal resolution of the determination of isopycnal displacements, as it took place at a scale significantly smaller than the scale of the displacements, set by the horizontal size of about 100 pixels of the sphere.

Figure 20 illustrates how the above procedure has been applied. Vertical interpolation by cubic splines has been used to transform the discrete measurement of the isopycnal displacements at the dye planes into a continuous measurement of the displacements through the column of fluid. Over 20 periods, slow but detectable noise was visible in these measurements, arising both from the drift in electronic equipment and the physical drift of the dye planes. In order to filter it, it was chosen to plot the differential displacement $\Delta \zeta(t)=\zeta(t+\Delta t)-\zeta(t)$ between two successive images taken with time increment $\Delta t=1 \mathrm{~s}$. A similar filtering technique had been used in the past with time increment $\Delta t$ small compared with the period of oscillation $T$, so that the ratio $\Delta \zeta / \Delta t$ was in good approximation a temporal derivative $\partial \zeta / \partial t$; namely, $\Delta t / T=0.07$ for Sutherland et al. (1999), 0.008 for Sutherland \& Linden (2002) and 0.06 for Sutherland et al. (2003). Here, with $\Delta t / T=0.14$, the time increment is too large for the ratio to be considered a derivative.

A second source of measurement error is the fact that the waves are observed through a perturbed fluid, causing optical distortions between the laser sheet and the front wall of the tank. These can be estimated by combining the optical theory of Onu et al. (2003), Flynn et al. (2003) and Décamp et al. (2008) with the wave theory of $\S 2$; namely, by combining expression (5) of the light ray displacement in Décamp et al. (2008), with the first term in curly braces in its right-hand side dropped, with the expression of $\Delta N^{2}$ derived from (2.16c) above, and integrating the result between 
(a)

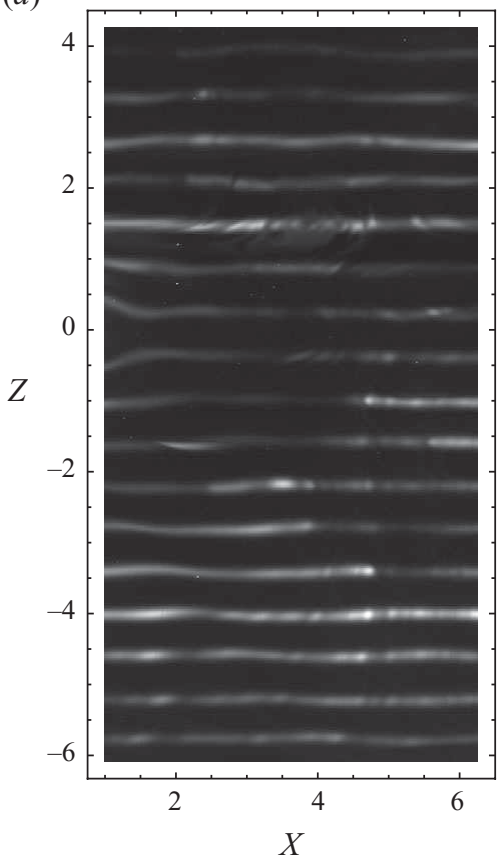

(b)

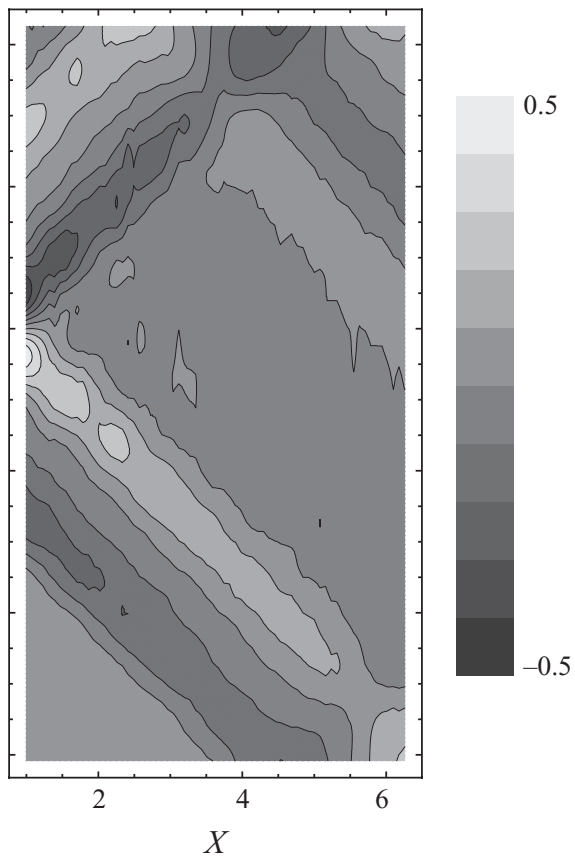

FIgURE 20. Analysis of the dye pattern from figure 18 on the right of the sphere, transforming (a) the corresponding portion of the pattern, 452 pixels wide by 889 pixels high, into $(b)$ a plot of the differential displacement $\Delta \zeta / A$ in the plane $Y=0$.

the front wall and the laser sheet. The optical distortions appear to be two orders of magnitude smaller than the vertical displacement of the dye planes. Accordingly, they can safely be neglected when processing the experimental data.

\section{Experimental measurements and comparison with theory}

Two series of experiments have been performed, one at small oscillation amplitude $K e=0.18$ so that linear theory applies, and the other at larger amplitude $K e=0.50$ so that nonlinear effects manifest themselves. Except when stated otherwise, all the other parameters were kept the same in both series.

\subsection{Linear regime}

At small amplitude $K e=0.18$, seven successive images were used for the large sphere, taken $1 \mathrm{~s}$ apart starting $0.38 \mathrm{~s}$ into the $21 \mathrm{st}$ period. Figure 18 reproduces the fourth image and figure 21 the associated theoretical prediction based on (2.16). The agreement between experiment and theory is good, but because of the smallness of the deformation of the dye lines, further fine-tuned processing by the cross-correlation technique of $\S 5.2$ is required for a quantitative comparison.

Four dye lines were selected, namely the third and fourth lines $Z=1.44$ and 2.08 above the midplane $Z=0$ and the sixth and seventh lines $Z=-3.48$ and -4.08 below it, respectively. The two upper lines were chosen so as to avoid the shadow of the sphere, visible in figure 21 and preventing measurement near the axis $X=0$. The two lower lines were chosen so as to reach larger vertical distances $|Z|$ than is possible above the sphere, owing to the reflections at the surface and bottom; these reflections, 


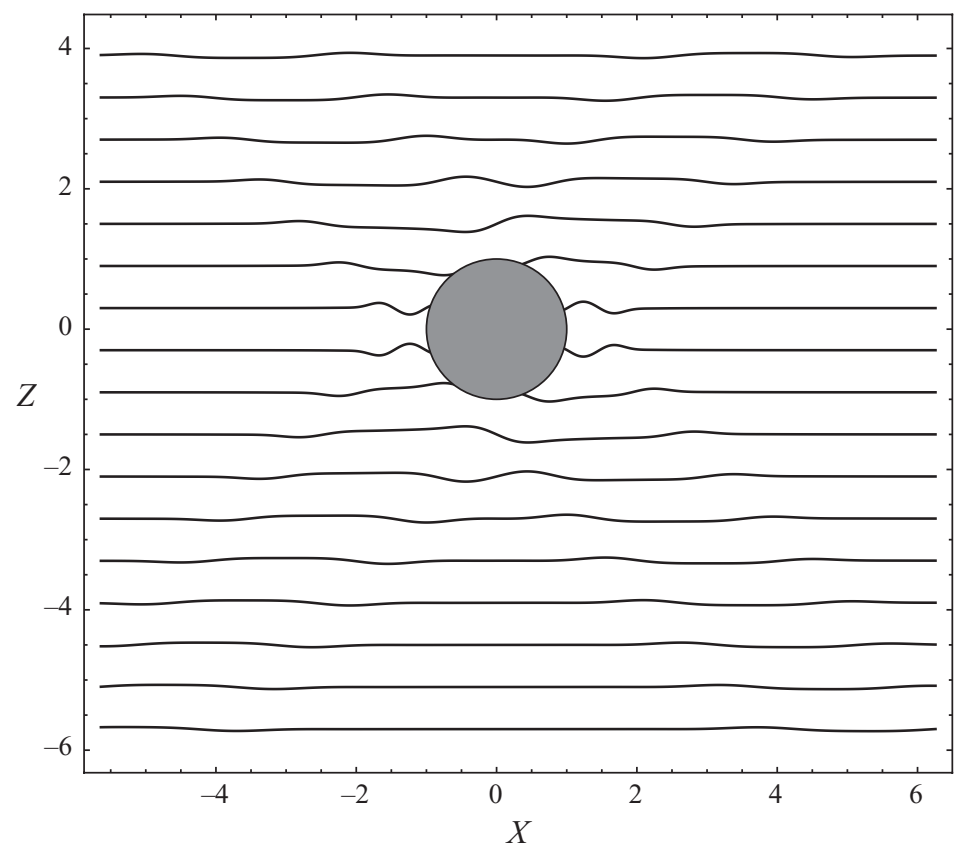

Figure 21. Theoretical dye pattern for figure 18. A close-up near the sphere was provided earlier in figure $7(b)$.

visible in figure $20(b)$, restrict the usable vertical range to, say, $-5<Z<2.5$, and the usable horizontal range to, say, $0<X<4$ for the two upper lines and $1<X<6$ for the two lower lines. The antisymmetry of the vertical displacement field with respect to the plane $Z=0$ was used to translate the measurements above this plane into measurements below it, so that the evolution of the wave profiles with $|Z|$ could be monitored.

The position of the dye lines in the transition diagram in figure 11(b) implies that the horizontal profiles are expected to be bimodal, consistent with the theoretical amplitude profiles in figure 8. This is verified by the experimental profiles in figure 22. A comparison with the exact theoretical prediction (2.16) and its farfield approximation (2.17) shows that only the former can account for the zero differential displacement observed at the axis $X=0$, and for the ratio between the major and minor peaks. Around $|Z|=3$ the far-field approximation becomes valid for the amplitude, while the phase remains slightly distorted by it.

Two dye lines at $Z=-5.8$ and -6.8 and five successive images starting $0.17 \mathrm{~s}$ into the 21st period were used for the small sphere. Consistent with figures 9 and $11(b)$, the experimental profiles in figure $23(a, c)$ are unimodal and compare excellently with the exact and far-field theoretical predictions, which are practically indistinguishable.

\subsection{Nonlinear regime}

Increasing the oscillation amplitude of the small sphere to $K e=0.50$ and monitoring the same dye lines on five successive images starting $0.20 \mathrm{~s}$ into the twenty-first period, the profiles in figure $23(b, d)$ are obtained. Their shape remains essentially unchanged, but their amplitude is overestimated by the theory by about $20 \%$.

This nonlinear effect called saturation, or its absence, has already been reported in the literature for oscillating cylinders and spheres, as listed in table 2. Saturation 


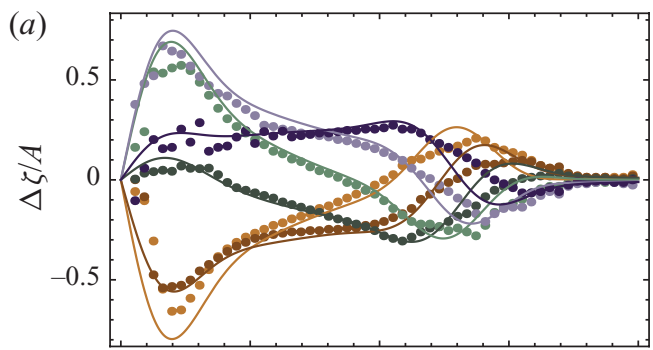

(b)
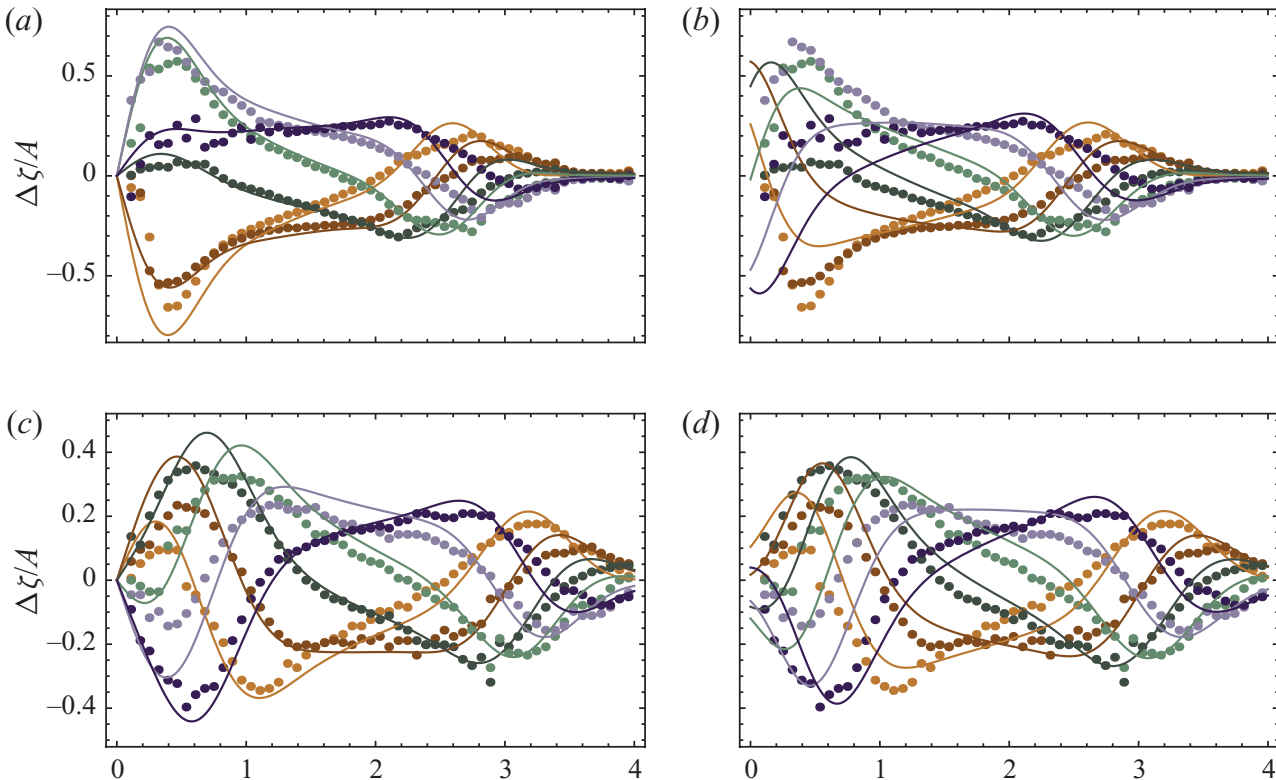

(d)
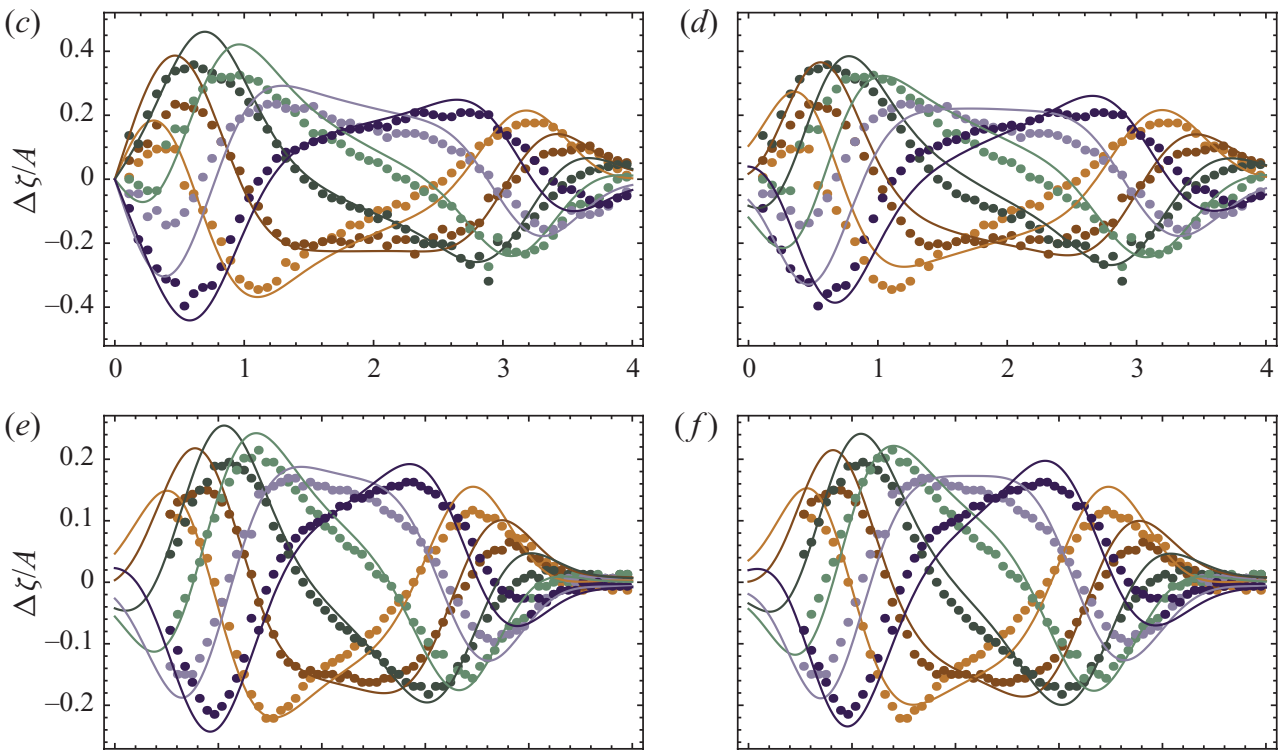

(f)
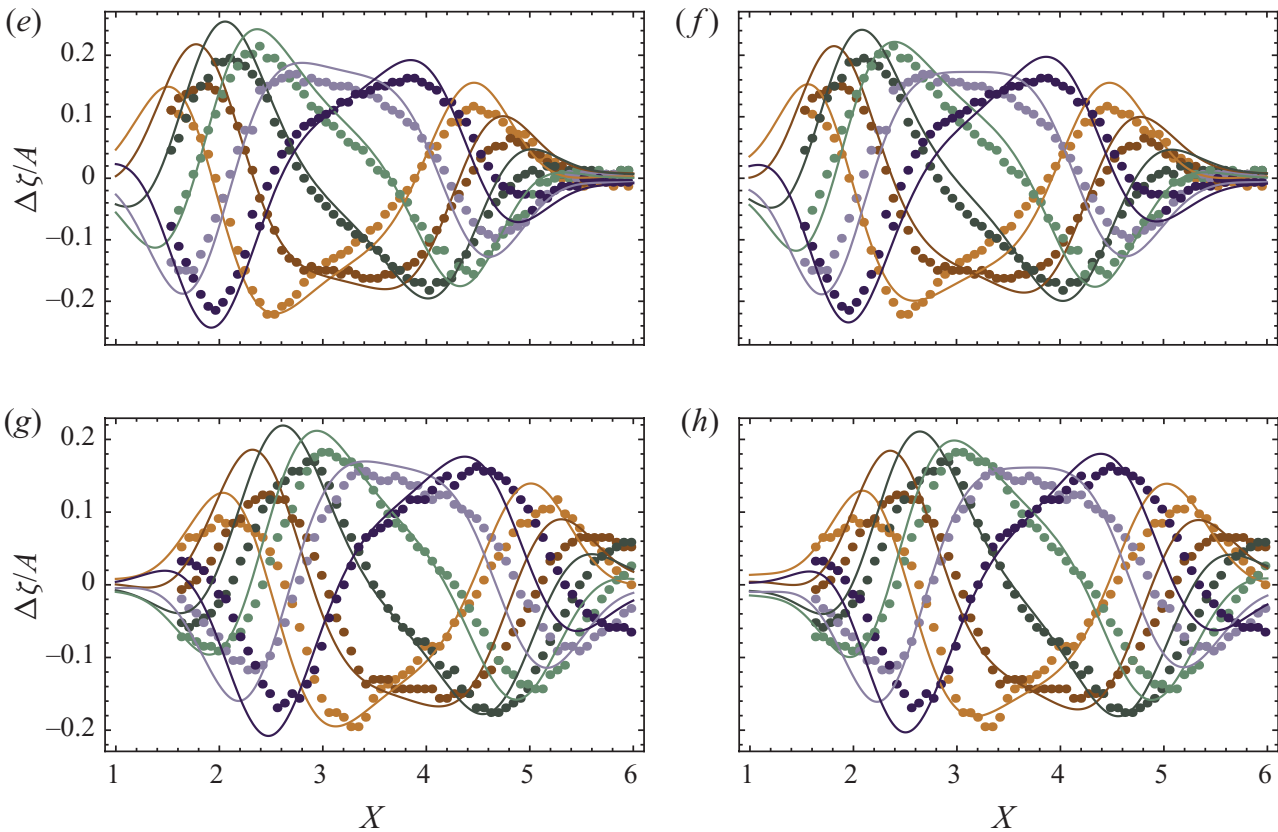

(h)

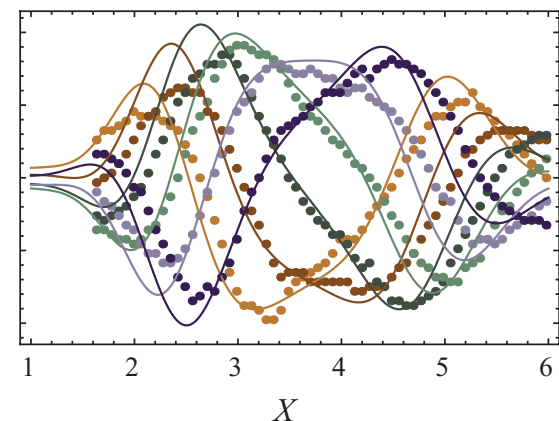

FIGURE 22. Horizontal profiles of the differential displacement at $(a, b) \quad Z=-1.44$, $(c, d) Z=-2.08,(e, f) Z=-3.48$ and $(g, h) Z=-4.08$, for the large sphere at $K e=0.18$. The lines represent the exact theory $(a, c, e, g)$ and the far-field theory $(b, d, f, h)$, and the dots the experimental data at successive times $0.38 \mathrm{~s}$ (light brown), $1.38 \mathrm{~s}$ (dark brown), $2.38 \mathrm{~s}$ (dark green), $3.38 \mathrm{~s}$ (light green), $4.38 \mathrm{~s}$ (light blue) and $5.38 \mathrm{~s}$ (dark blue) into the 21st period of oscillation.

appears to depend not only on the Keulegan-Carpenter number $K e$, but also on the Reynolds-Stokes number $R e$; specifically, saturation has only been observed for $R e>200$ and the associated threshold $K e_{c}$ varies with $R e$. 


\begin{tabular}{llrrr} 
& & & \multicolumn{2}{c}{ Ke } \\
\cline { 4 - 5 } Paper & Body & $R e$ & Linearity & Saturation \\
Peters (1985) & Cylinder & 74 & $0.42-0.94$ & \\
Peters (1985) & Cylinder & 110 & $0.22-0.56$ & \\
Peters (1985) & Cylinder & 130 & $0.24-0.62$ & \\
Makarov et al. $(1990)$ & Cylinder & 8 & $0.03-0.67$ & \\
Sutherland et al. $(1999)$ & Cylinder & 260 & 0.13 & 0.19 \\
Sutherland \& Linden (2002) & Cylinder & 140 & $0.11-0.19$ & \\
Zhang et al. $(2007)$ & Cylinder & 1300 & $0.05-0.15$ & $0.15-0.20$ \\
Ermanyuk \& Gavrilov (2008) & Cylinder & 99 & $0.30,0.60,0.90$ & \\
Flynn et al. $(2003)$ & Sphere & 350 & $0.15,0.19$ & 0.26 \\
King et al. $(2009)$ & Sphere & 2000 & $0.05-0.30$ & $0.30-0.60$ \\
Present paper & Sphere & 520 & 0.18 & 0.50
\end{tabular}

TABLE 2. Observations of wave amplitude versus oscillation amplitude in the literature.

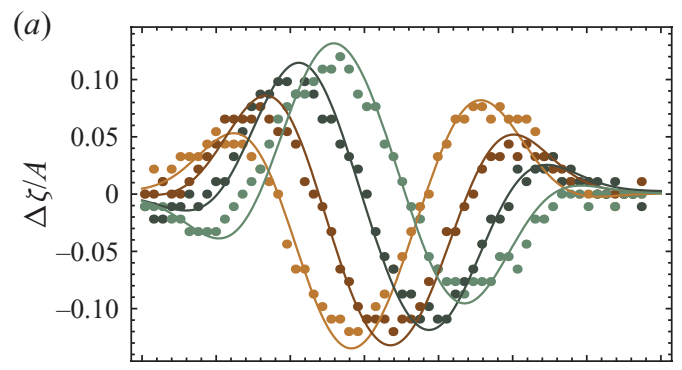

(b)
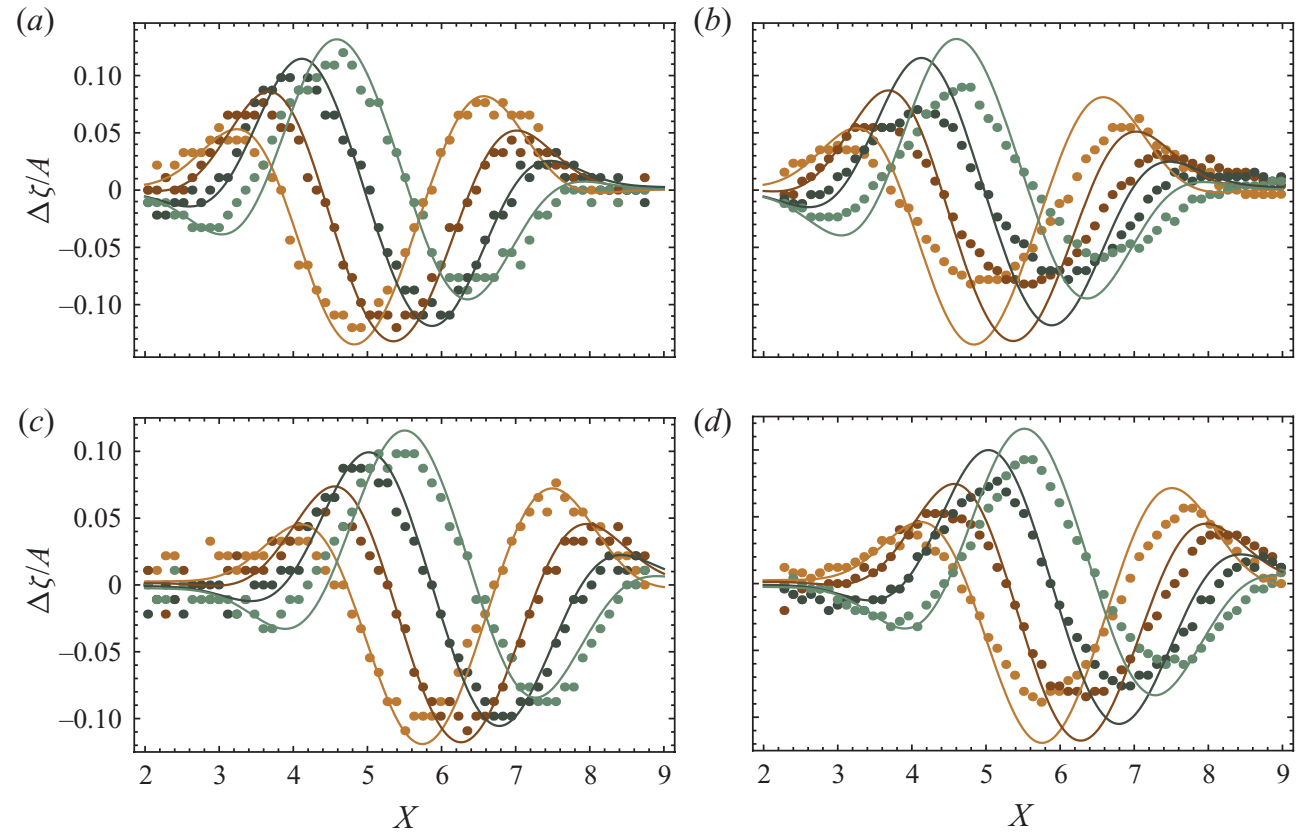

FIGURE 23. Horizontal profiles of the differential displacement at $(a, b) \quad Z=-5.8$ and $(c, d) Z=-6.8$, for the small sphere at $(a, c) K e=0.18$ and $(b, d) K e=0.50$. The lines represent the exact theory, and the dots the experimental data at successive times $0.17 \mathrm{~s}$ (light brown), $1.17 \mathrm{~s}$ (dark brown), $2.17 \mathrm{~s}$ (dark green) and $3.17 \mathrm{~s}$ (light green) for $K e=0.18$, and $0.20 \mathrm{~s}$ (light brown), $1.20 \mathrm{~s}$ (dark brown), $2.20 \mathrm{~s}$ (dark green) and $3.20 \mathrm{~s}$ (light green) for $\mathrm{Ke}=0.50$, into the 21st period of oscillation.

On the basis of the observations of Ermanyuk \& Gavrilov (2008) for the circular oscillations of a horizontal cylinder in the vertical plane, saturation seems to be caused by the loss of wave energy to mixing in the vicinity of the oscillating body. For vertical rectilinear oscillations, Ivanov (1988) observed that mixing close to the cylinder was related to the alternative Reynolds number,

$$
R e_{s}=\operatorname{Re} K e^{2}=\frac{2 \omega A^{2}}{v},
$$



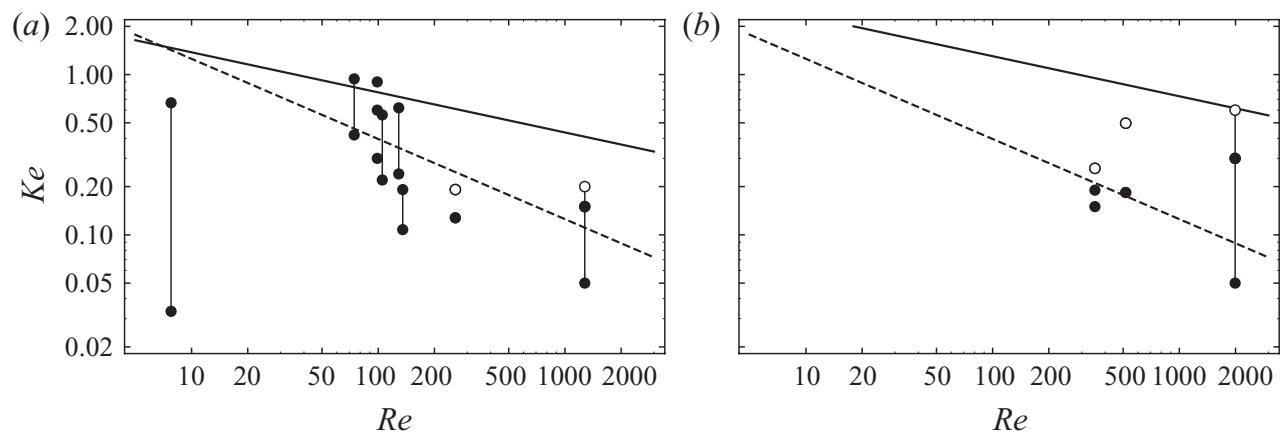

FIGURE 24. Instability thresholds of the oscillatory boundary layer at $(a)$ a circular cylinder and $(b)$ a sphere. The dots represent the observations of linear variation $(\bullet)$ and saturation $(\bigcirc)$ of the wave amplitude according to table 2. The lines represent the two alternative thresholds (6.3), based on the linear theory of Hall (1984) and Otto (1992) for a homogeneous fluid (solid lines), and (6.2), based on the observations of Ivanov (1988) in a stratified fluid (dashed lines) applied for definiteness not only to the cylinder but also to the sphere.

in which the radius of the cylinder is replaced by the amplitude of oscillation, and that for $R e_{s}<5 \pi \approx 16$ the flow was fully attached while for $R e_{s}>16$ vorticity generation and mixing took place. A first value of the saturation threshold follows,

$$
K e_{c}=\frac{4}{R e^{1 / 2}} \quad \text { (circular cylinder). }
$$

Now, the Reynolds number $R e_{s}$ is known to characterize, in a homogeneous fluid, the steady streaming flow formed, in an outer boundary layer of the thickness $(2 v / \omega)^{1 / 2} a / A$, by the Reynolds stresses in the inner Stokes boundary layer of the thickness $(2 v / \omega)^{1 / 2}$; see e.g. Batchelor $(1967, \S 5.13)$ and Riley (2001). In the approximation $R e \gg 1$, the linear stability analysis of the outer layer yields a critical value of $R e_{s}$ given by $6.00 \mathrm{Ke}^{1 / 2}$ for the cylinder (Hall 1984) and $16.96 \mathrm{Ke}^{1 / 2}$ for the sphere (Otto 1992). On the assumption that these values remain unchanged in a stratified fluid, a second, alternative value of the saturation threshold follows,

$$
K e_{c}=\frac{2.45}{R e^{1 / 4}} \quad \text { (circular cylinder), } \quad \frac{4.12}{R e^{1 / 4}} \quad \text { (sphere). }
$$

This assumption is supported by the flow regime diagrams in the $(R e, K e)$-plane drawn, in a stratified fluid, by Lin et al. (1994) for the horizontal oscillations of a sphere and $\mathrm{Xu}$ et al. (1997) for the horizontal oscillations of a cylinder; namely, the boundaries between fully attached flow and attached vortices for the sphere, and weakly detached flow and localized mixing for the cylinder, correspond approximately to the second threshold. As the oscillation amplitude increases over the threshold, the visualizations of Chashechkin \& Prikhod'ko (2007) and Chashechkin (2007) for the vertical oscillations of a sphere seem to indicate that vorticity generation and mixing escape from the streaming layer and invade the whole overlap region of the wave beams, ultimately transforming it into jets aligned along the direction of oscillation.

Figure 24 plots together the observations of linear amplitude variation and saturation and the two threshold predictions in the (Re, Ke)-plane. The observations of saturation are too scarce to draw definite conclusions, all the more so since saturation was not studied per se in most cases, so that the analysis in table 2 is mostly a reinterpretation of the observations; in particular, saturation is reported by 
Sutherland et al. (1999) and Flynn et al. (2003) to be fairly weak and possibly fall within the experimental noise. Accordingly, nonlinear scaling is still an open question.

\section{Energy radiation and application to internal tides}

Having validated linear theory, we return to the original motivation of our study, namely internal tides. In this context, the quantity of interest is the average radiated power, characterizing the conversion rate of barotropic energy into internal tides. Only the steady inviscid limit has been considered in the literature, often, but not always, on the hydrostatic approximation. Various topographies have been considered, either two- or three-dimensional and either flat or upright. Combining the results, the conversion rate is seen to scale as $\left(N^{2}-\omega^{2}\right)^{1 / 2} h^{2}|U|^{2}$ in two dimensions and $\left(N^{2}-\omega^{2}\right)^{1 / 2} \ell h^{2}|U|^{2}$ in three dimensions, with $U$ as the velocity amplitude of the barotropic flow, $h$ as the height of the topography and $\ell$ as its half-width; see e.g. Bell (1975a,b), Llewellyn Smith \& Young (2002, 2003), Pétrélis et al. (2006), Nycander (2006) and Bühler \& Muller (2007).

The average radiated power $P$ may be calculated by integrating the average rate of energy flux, $\boldsymbol{I}=\operatorname{Re}\left[p \boldsymbol{u}^{*}\right] / 2$ with $p$ as the perturbation pressure and * as a complex conjugate, over a biconical surface crossing the wave beams at a given distance $\left|z_{ \pm}\right|$. The integration is performed in the far field, implying that, for steady inviscid waves, the non-integrable singularities in the overlap region of the wave beams are avoided and only the integrable singularities at the beam edges remain; accordingly, the steady inviscid limits $\alpha \rightarrow 0$ and $\beta \rightarrow 0$ are uniform. Combining the three results (4.42), (5.19) and (6.14) of Voisin (2003), we obtain

$$
P=\frac{\rho_{0} N \cos \theta \sin \theta}{32 \pi^{2}} \int_{0}^{2 \pi} \mathrm{d} \varphi_{k} \sum_{ \pm} \int_{0}^{1 /\left(\alpha\left|z_{ \pm}\right|\right)} \mathrm{d} k_{ \pm} \exp \left(-2 \beta k_{ \pm}^{3}\left|z_{ \pm}\right|\right)\left|q_{ \pm}\left(k_{ \pm}, \varphi_{k}, 0\right)\right|^{2},
$$

with no need of the hydrostatic approximation.

For the sphere, represented by the source (2.10) of the spectrum (2.11), this becomes, in the steady inviscid case,

$$
P_{0}=\frac{\pi^{2}}{3} \rho_{0}\left(N^{2}-\omega^{2}\right)^{1 / 2} a^{3} \frac{\omega}{N}\left[|U|^{2} \frac{2 \omega^{2} / N^{2}}{|1+B(\omega / N)|^{2}}+|W|^{2} \frac{1-\omega^{2} / N^{2}}{|1-B(\omega / N)|^{2}}\right],
$$

and evaluates otherwise to

$$
\frac{P}{P_{0}}=\frac{6}{\pi} \int_{0}^{1 / \sigma_{u}} \exp \left(-2 \sigma_{v}^{3} K_{ \pm}^{3}\right) j_{1}^{2}\left(K_{ \pm}\right) \mathrm{d} K_{ \pm},
$$

implying that the parameters $\sigma_{u}$ and $\sigma_{v}$ provide an adequate characterization of unsteady and viscous effects on the conversion rate.

For internal tides, we set $W=0$. The sphere translates to a hemisphere in a flow of the velocity $-\boldsymbol{U} \mathrm{e}^{-\mathrm{i} \omega t}$, with $\boldsymbol{U}=(U, 0,0)$, and the source (2.10) represents the combination of the hemisphere and its image through the plane ocean bottom $z=0$. In order to study the effect of the criticality of the topography, we consider not only the supercritical hemisphere but also flat topographies of a slope much smaller than the slope of the wave rays; namely, we consider topographies of the elevation $f\left(\boldsymbol{x}_{h}\right)$, with $\boldsymbol{x}_{h}=(x, y)$, satisfying $h / \ell \ll \omega / N$ with $h$ as the maximum value of $f\left(\boldsymbol{x}_{h}\right)$ and $\ell$ its scale of variation. Such topographies are subcritical by nature, and may be 
(a)

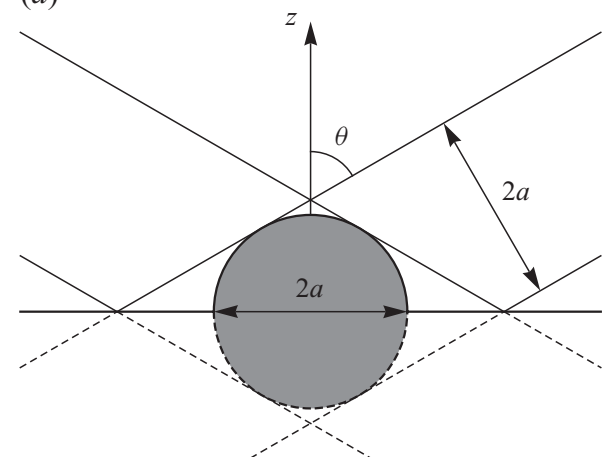

(b)

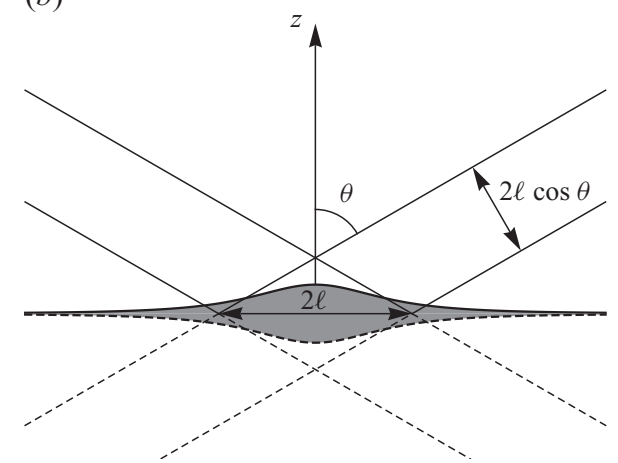

FiguRE 25. Beam geometry for internal tides from $(a)$ a hemisphere and $(b)$ a flat topography (for definiteness a witch of Agnesi). For the hemisphere, the beam width is set by the critical rays tangent to the topography and its image through the bottom. For flat topography, the beam width is set by the rays emanating from the base of the topography.

represented by the source

$$
q(\boldsymbol{x})=-2 U \frac{\partial}{\partial x} f\left(\boldsymbol{x}_{h}\right) \delta(z), \quad q(\boldsymbol{k})=-2 \mathrm{i} U k f\left(\boldsymbol{k}_{h}\right),
$$

where $\boldsymbol{k}_{h}=(k, l)$; see e.g. Voisin (2007) in the context of lee waves. In the steady inviscid case, the power radiated in the half-space $z>0$ is obtained immediately as

$$
P_{0}=\frac{\rho_{0}\left(N^{2}-\omega^{2}\right)^{1 / 2}|U|^{2}}{8 \pi^{2}} \iint \frac{k^{2}}{\kappa_{h}}\left|f\left(\boldsymbol{k}_{h}\right)\right|^{2} \mathrm{~d}^{2} k_{h},
$$

where $\kappa_{h}=\left|\boldsymbol{k}_{h}\right|$, consistent with Bell (1975b), Llewellyn Smith \& Young (2002) and Bühler \& Muller (2007).

The presence of the spectrum $f\left(\boldsymbol{k}_{h}\right)$ indicates that the structure of the internal tide is set by the horizontal scale $\ell$ of the topography. As illustrated in figure 25, the natural wave scale is the beam half-width $\ell \cos \theta$ and the natural non-dimensionalization of the coordinates is $\left(X_{+}, Z_{+}\right)=\left(x_{+}, z_{+}\right) /(\ell \cos \theta)$ in real space and $K_{+}=k_{+} \ell \cos \theta$ in Fourier space. When unsteady and viscous effects come into play, so does the Reynolds-Stokes number

$$
R e=\frac{2 \omega^{3} \ell^{2}}{N^{2} v}
$$

yielding for the parameters $\sigma_{u}$ and $\sigma_{v}$ the same final expressions as for the hemisphere, namely

$$
\sigma_{u}=\frac{Z_{+}}{\omega t \tan \theta}, \quad \sigma_{v}=\left(\frac{Z_{+}}{R e \tan \theta}\right)^{1 / 3} .
$$

The conversion rate becomes

$$
P=\frac{\rho_{0}\left(N^{2}-\omega^{2}\right)^{1 / 2}|U|^{2}}{8 \pi^{2} \ell^{3}} \int_{0}^{2 \pi} \mathrm{d} \varphi_{k} \cos ^{2} \varphi_{k} \int_{0}^{1 / \sigma_{u}} \mathrm{~d} K_{+} \exp \left(-2 \sigma_{v}^{3} K_{+}^{3}\right) K_{+}^{2}\left|f\left(\boldsymbol{k}_{h}\right)\right|^{2},
$$

with

$$
\boldsymbol{k}_{h}=\frac{K_{+}}{\ell}\left(\cos \varphi_{k}, \sin \varphi_{k}\right) .
$$


The most frequent examples of seafloor topography, considered e.g. by Llewellyn Smith \& Young (2002), are the witch of Agnesi

$$
f\left(\boldsymbol{x}_{h}\right)=h\left(1+\frac{r_{h}^{2}}{\ell^{2}}\right)^{-3 / 2}, \quad f\left(\boldsymbol{k}_{h}\right)=2 \pi h \ell^{2} \exp \left(-\kappa_{h} \ell\right),
$$

and the Gaussian bump

$$
f\left(\boldsymbol{x}_{h}\right)=h \exp \left(-\frac{r_{h}^{2}}{2 \ell^{2}}\right), \quad f\left(\boldsymbol{k}_{h}\right)=2 \pi h \ell^{2} \exp \left(-\frac{\kappa_{h}^{2} \ell^{2}}{2}\right),
$$

of the same volume $2 \pi h \ell^{2}$. The associated conversion rates are, for the former,

$$
\begin{gathered}
P_{0}=\frac{\pi}{8} \rho_{0}\left(N^{2}-\omega^{2}\right)^{1 / 2} \ell h^{2}|U|^{2}, \\
\frac{P}{P_{0}}=4 \int_{0}^{1 / \sigma_{u}} \exp \left(-2 \sigma_{v}^{3} K_{ \pm}^{3}\right) K_{+}^{2} \exp \left(-2 K_{+}\right) \mathrm{d} K_{+},
\end{gathered}
$$

and for the latter,

$$
\begin{gathered}
P_{0}=\frac{\pi^{3 / 2}}{8} \rho_{0}\left(N^{2}-\omega^{2}\right)^{1 / 2} \ell h^{2}|U|^{2} \\
\frac{P}{P_{0}}=\frac{4}{\pi^{1 / 2}} \int_{0}^{1 / \sigma_{u}} \exp \left(-2 \sigma_{v}^{3} K_{ \pm}^{3}\right) K_{+}^{2} \exp \left(-K_{+}^{2}\right) \mathrm{d} K_{+} \cdot
\end{gathered}
$$

We compare these rates for all three topographies, namely the hemisphere, the witch of Agnesi and the Gaussian bump.

\subsection{Steady inviscid case}

Consider the steady inviscid case first. For flat topographies, the conversion rate exhibits the anticipated dependence as $\left(N^{2}-\omega^{2}\right)^{1 / 2} \ell h^{2}|U|^{2}$, implying a maximum at the frequency ratio $\omega / N=0$ at the fixed velocity amplitude $U$ and $\omega / N=\sqrt{ }(2 / 3) \approx 0.82$ at the fixed excursion $A=|U| / \omega$. For the hemisphere, the frequency dependence is more complicated and the conversion rate is a maximum at $\omega / N=0.71$ at fixed $U$ and 0.85 at fixed $A$.

In the more general situation, relevant to the laboratory, of a sphere oscillating at an arbitrary angle $\eta$ to the horizontal, the variations of the radiated power with frequency are plotted at the fixed excursion in figure 26. They are compared with those for a circular cylinder, based on the calculation of the radiated power by Hurley (1997), namely

$$
P_{0}=\frac{\pi}{2} \rho_{0}\left(N^{2}-\omega^{2}\right)^{1 / 2} a^{2}\left(|U|^{2}+|W|^{2}\right),
$$

independent of $\eta$ and exhibiting the same frequency dependence as for flat topographies. The variations are similar for the cylinder and the sphere, and are consistent with the experimental measurements by Ermanyuk \& Gavrilov (2002, 2003). In particular, the radiated power for the cylinder is at its maximum at frequency ratio 0.82 independent of $\eta$, and for the sphere varying weakly with it, between 0.83 and 0.85 .

This latter result has interesting implications in another area, namely internal wave generation by turbulence. It has been observed in several circumstances that a region of turbulent motion in a stratified fluid generates internal waves with a well-defined spectrum, peaked at frequency ratio 0.8 for a two-dimensional mixed region released at its neutral buoyancy level (Wu 1969), 0.7 when the release takes place above this 

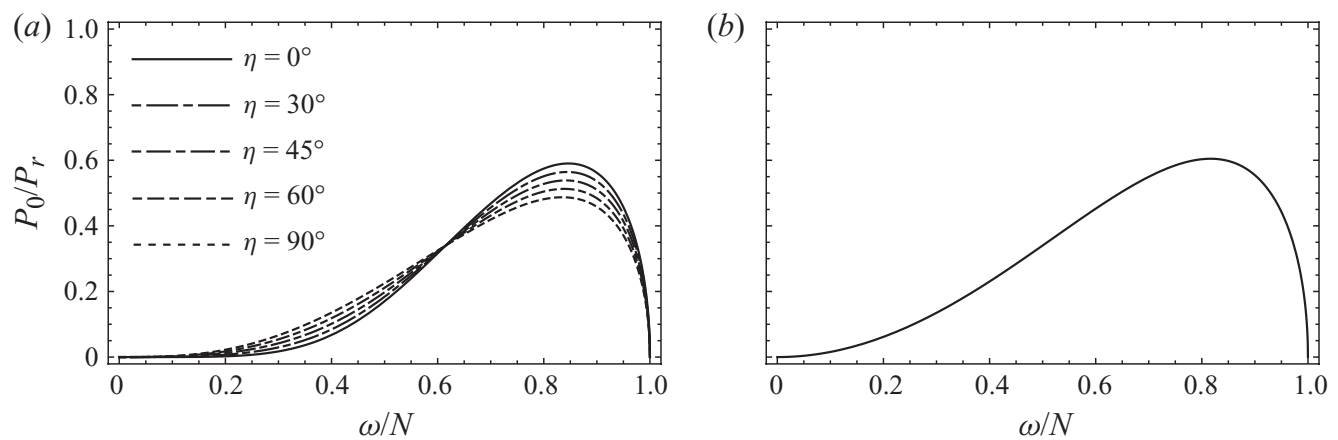

FIGURE 26. Frequency dependence of the steady inviscid power output of $(a)$ a sphere oscillating at varying angle $\eta$ to the horizontal and $(b)$ a circular cylinder, normalized by $P_{r}=\rho_{0} N^{3} a^{3} A^{2}$ for the sphere and $\rho_{0} N^{3} a^{2} A^{2}$ for the cylinder, with $A=\left(|U|^{2}+|W|^{2}\right)^{1 / 2} / \omega$ the excursion. For internal tides, the steady inviscid conversion rate varies according to $(a)$ with $\eta=0^{\circ}$ for a hemisphere and $(b)$ for a semi-circular cylinder; for flat topography, the frequency dependence is the same as in $(b)$.
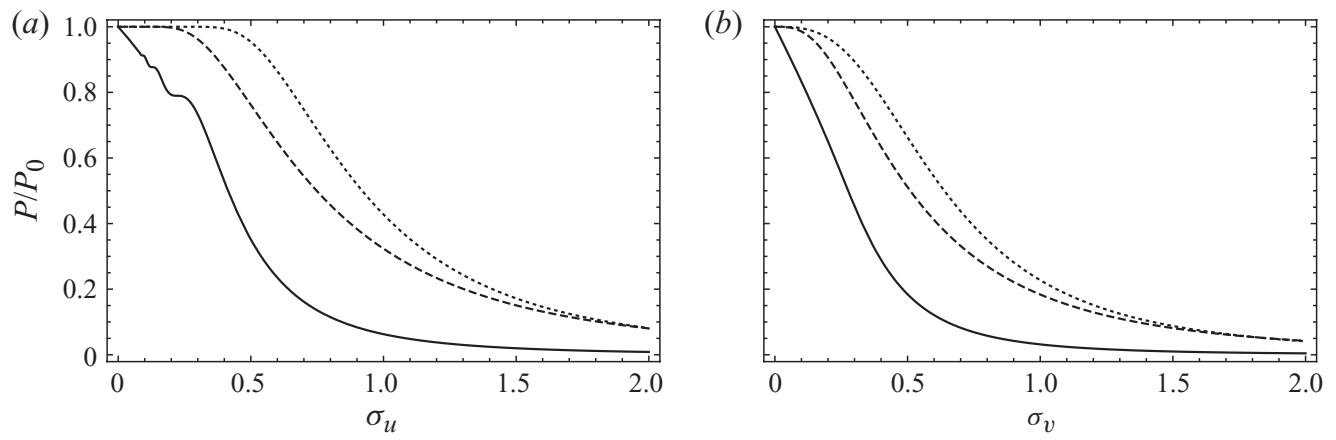

FiguRE 27. Power decay owing to $(a)$ unsteadiness alone and $(b)$ viscosity alone, for the hemisphere (solid lines), the witch of Agnesi (dashed lines) and the Gaussian bump (dotted lines).

level (Cerasoli 1978) and 0.5 for a freely decaying three-dimensional turbulent field (Riley, Metcalfe \& Weissman 1981). If a turbulent region can be thought of as an incoherent assembly of turbulent patches oscillating at random frequency in random directions with all about the same excursion, then the existence of a maximum of radiation at a fixed frequency independent of the direction of oscillation might account for the presence of the peak.

\subsection{Unsteady and viscous dissipation}

Coming back to internal tides, we consider the unsteady and viscous decay of the conversion rate. Figure 27 illustrates this decay in the limits $\sigma_{v} \rightarrow 0$ and $\sigma_{u} \rightarrow 0$, corresponding to unsteadiness and viscosity acting in isolation, respectively, for the hemisphere, the witch of Agnesi and the Gaussian bump. The associated asymptotics are given in table 3. The decay is initially faster for the hemisphere. Ultimately it becomes identical for the three, as $1 / \sigma_{u}^{3}$ i.e. $1 /\left|Z_{ \pm}\right|^{3}$ when unsteady effects are dominant, and $1 / \sigma_{v}^{3}$ i.e. $1 /\left|Z_{ \pm}\right|$when viscous effects are dominant, corresponding to such large distances that the size of the topography is insignificant and only the multipolar order of the forcing matters, a situation described in table 3 of Voisin (2003). 


\begin{tabular}{lccccccc} 
& \multicolumn{3}{c}{$\sigma_{v}=0$} & & \multicolumn{2}{c}{$\sigma_{u}=0$} \\
\cline { 2 - 3 } Topography & $\left.\frac{P}{P_{0}}\right|_{\sigma_{u} \ll 1}$ & $\left.\frac{P}{P_{0}}\right|_{\sigma_{u} \gg 1}$ & $\sigma_{u_{d}}$ & & $\left.\frac{P}{P_{0}}\right|_{\sigma_{v} \ll 1}$ & $\left.\frac{P}{P_{0}}\right|_{\sigma_{v} \gg 1}$ & $\sigma_{v_{d}}$ \\
Hemisphere & $1-\frac{3}{\pi} \sigma_{u}$ & $\frac{2}{9 \pi \sigma_{u}^{3}}$ & 0.41 & & $1-\frac{3}{\pi} 2^{1 / 3} \Gamma\left(\frac{2}{3}\right) \sigma_{v}$ & $\frac{1}{9 \pi \sigma_{v}^{3}}$ & 0.28 \\
Witch & $1-2 \frac{\exp \left(-2 / \sigma_{u}\right)}{\sigma_{u}^{2}}$ & $\frac{4}{3 \sigma_{u}^{3}}$ & 0.75 & $1-15 \sigma_{v}^{3}$ & $\frac{2}{3 \sigma_{v}^{3}}$ & 0.51 \\
Gaussian & $1-\frac{2}{\pi^{1 / 2}} \frac{\exp \left(-1 / \sigma_{u}^{2}\right)}{\sigma_{u}}$ & $\frac{4}{3 \pi^{1 / 2} \sigma_{u}^{3}}$ & 0.92 & & $1-\frac{8}{\pi^{1 / 2}} \sigma_{v}^{3}$ & $\frac{2}{3 \pi^{1 / 2} \sigma_{v}^{3}}$ & 0.64
\end{tabular}

TABLE 3. Asymptotic power decay owing to unsteadiness alone (left column) and viscosity alone (right column), and associated dissipation thresholds.

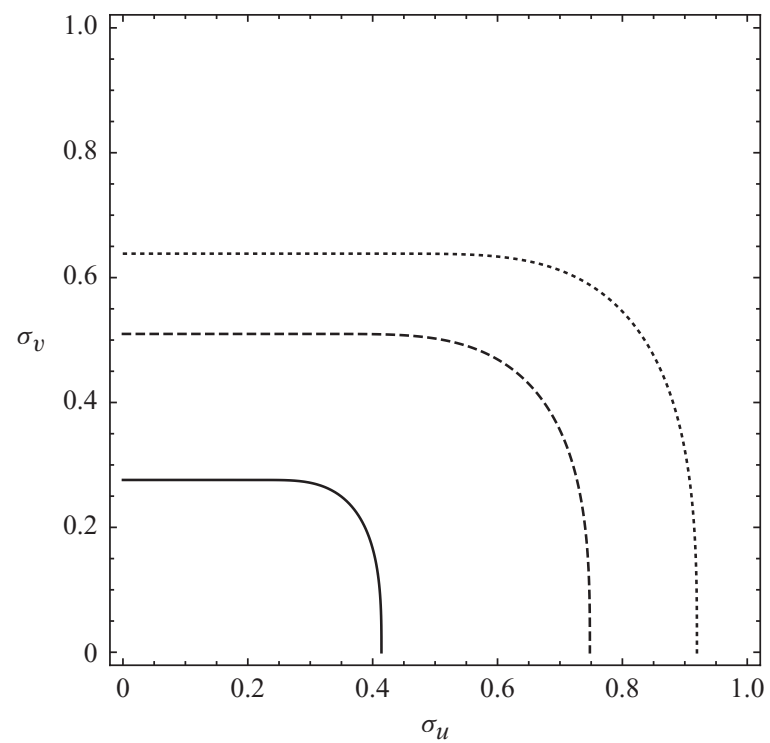

FIGURE 28. Dissipation threshold for the hemisphere (solid line), the witch of Agnesi (dashed line) and the Gaussian bump (dotted line).

Since the rate of decay varies with distance, another quantity must be introduced to characterize the dissipation of energy. We define a dissipation distance $Z_{d}$ as the distance where the radiated energy has been divided by two, namely

$$
\left.\frac{P}{P_{0}}\right|_{\left|Z_{ \pm}\right|=Z_{d}}=\frac{1}{2} .
$$

As for the transition from bimodality to unimodality in (3.12), this condition defines a boundary in the $\left(\sigma_{u}, \sigma_{v}\right)$-plane, plotted in figure 28 . The associated thresholds $\sigma_{u_{d}}$ and $\sigma_{v_{d}}$ are slightly larger than the transition thresholds $\sigma_{u_{c}}$ and $\sigma_{v_{c}}$ in figure 11. Plotting in figure 29(a) the energy decay with distance in the experiments of Flynn et al. (2003), King et al. (2009) and in the present experiments, all the measurements are seen to have taken place before the dissipation thresholds. 

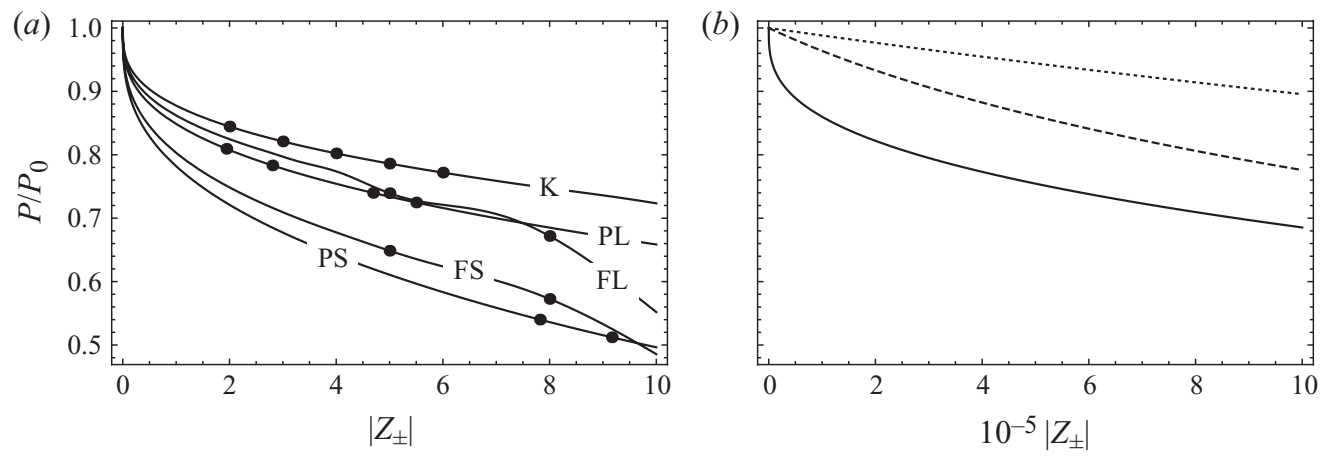

FiguRE 29. Power decay in $(a)$ existing laboratory experiments for the sphere, with the same notations as in figure 11, and (b) typical oceanic conditions for the hemisphere (solid line), the witch of Agnesi (dashed line) and the Gaussian bump (dotted line).

\subsection{Application to oceanic parameters}

In order to assess the relevance of these concepts for the ocean, we borrow the values of the buoyancy frequency $N=5 \times 10^{-4} \mathrm{~s}^{-1}$, the barotropic frequency $\omega=2 \times 10^{-4} \mathrm{~s}^{-1}$ and the velocity amplitude $U=1.4 \mathrm{~cm} \mathrm{~s}^{-1}$ from Llewellyn Smith \& Young (2002), and the turbulent kinematic viscosity $v=10 \mathrm{~mm}^{2} \mathrm{~s}^{-1}$ from Di Lorenzo, Young \& Llewellyn Smith (2006). For the witch of Agnesi and the Gaussian bump, we borrow the values of the height $h=320 \mathrm{~m}$ and the half-width $\ell=1.6 \mathrm{~km}$ from Llewellyn Smith \& Young (2002), who deduced them from seamount statistics in the Pacific. For the hemisphere, the radius $a=\left(3 h \ell^{2}\right)^{1 / 3} \approx 1300 \mathrm{~m}$ is chosen such that the volume of all three topographies is the same. Unsteady effects, difficult to quantify in the ocean, are ignored.

The smallness of the Keulegan-Carpenter numbers, of the order of $10^{-2}$, legitimates the linear approach. The inviscid radiated power $P_{0}$ is of the order of $10 \mathrm{~kW}$ for the flat topographies, consistent with Llewellyn Smith \& Young (2002), and one order of magnitude larger for the hemisphere. The hugeness of the Reynolds-Stokes numbers, of orders $10^{7}$ for the flat topographies and $10^{8}$ for the hemisphere, leaves no room for viscosity to dissipate this power; for example, the dissipation thresholds in figure 28 translate to dimensional distances of order $10^{9} \mathrm{~m}$. Figure $29(\mathrm{~b})$ represents the power decay with distance, implying that $10 \%$ decay requires propagation over non-dimensional distances of about $5 \times 10^{4}$ for the hemisphere, $3 \times 10^{5}$ for the witch of Agnesi and $10^{6}$ for the Gaussian bump, corresponding to dimensional distances of order $10^{8} \mathrm{~m}$, larger than the circumference of the Earth $4 \times 10^{7} \mathrm{~m}$ ! For the same reason, we do not expect the transition from bimodality to unimodality to be observed in the ocean for supercritical topography.

\section{Conclusions}

A joint theoretical and experimental study has been presented on the generation of internal gravity waves by an oscillating sphere. The theory considered oscillations at an arbitrary angle to the horizontal, by superposition of horizontal and vertical oscillations, while the experiments involved horizontal oscillations. The theory was applied to the calculation of the conversion rate of barotropic tidal energy into internal tides, for several seafloor topographies either hemispherical or flat. 
Experimentally, the dye-line technique of Hopfinger et al. (1991) for the measurement of isopycnal displacements has been developed and automated, providing both high-precision data, with sub-pixel accuracy, and readily interpretable images of the flow. Use has been made of the radiation pattern of the waves to effectively prevent reflection at the tank walls. The dye-line technique has been applied to the measurement of the waves in the vertical plane along the direction of oscillation through the centre of the sphere. A separate paper (Ermanyuk et al. 2010) will present the three-dimensional reconstruction of the wave field.

Theoretically, the boundary integral method has been shown to provide an adequate representation of the sphere, satisfying the free-slip boundary condition at its surface. In order to reproduce the variety of experimental configurations here and in the literature, near-field effects, viscous effects and the unsteady effects arising from the interference with transients generated at the start-up must all be taken into account. Near-field effects are significant within 1 diameter above the top or below the bottom of the sphere. Two dimensionless parameters have been introduced to quantify unsteady and viscous effects, and a transition diagram has been drawn in the two-parameter plane to separate an inner bimodal zone, with bimodal wave profiles, and an outer unimodal zone, with unimodal profiles. Bimodal profiles have steady inviscid singularities at the critical rays tangent to the sphere, with unsteady and viscous effects acting locally at these rays to keep the waves finite. Unimodal profiles are governed primarily by unsteady and viscous effects. The parameter quantifying viscous effects derives from that introduced by Hurley \& Keady (1997) for a cylinder. Unsteady effects become negligible after a time depending on the extent of the wave field considered; under typical experimental conditions, this time varies between 5 and 10 oscillation periods.

At small oscillation amplitude, of 0.2 times the sphere radius, the waves are linear and the agreement between experiment and theory is excellent. Increasing the amplitude to a higher value, of 0.5 times the radius, gives rise to nonlinear effects which manifest themselves through a saturation of the wave amplitude, $20 \%$ smaller than its linear prediction, owing to the loss of wave energy to mixing at the sphere. The choice of an oscillation frequency above half the buoyancy frequency prevents the propagation of higher harmonics, which will be considered in Ermanyuk et al. (2010).

The application of the theory to internal tides revealed several interesting features. The existence of bimodal profiles, and the transition to unimodal profiles with distance, are specific to supercritical topographies, of slope locally larger than the slope of the rays. For subcritical topographies, of slope everywhere smaller than the ray slope, the profiles are always unimodal. The conversion rate of barotropic tidal energy into internal tides scales as $\left(N^{2}-\omega^{2}\right)^{1 / 2} \ell h^{2} U^{2}$ for flat topographies, of infinitesimal slope, with $N$ as the buoyancy frequency, $\omega$ as the frequency and $U$ as the velocity amplitude of the barotropic flow, and $h$ as the height and $\ell$ as the half-width of the topography; for the hemisphere, the dependence on $\omega / N$ is more complicated. Unsteady and viscous effects induce a decay of the conversion rate with distance, but in oceanic conditions the decay is so slow that it is unlikely to be observed. Similarly, the transition from bimodality to unimodality takes place at such large distances in the ocean that it is unlikely to be observed.

A remarkable feature of the solution for the sphere, pointed out by a referee, is the simple form of the surface source distribution (2.10) equivalent to the sphere: in order to account for the singularity of the waves at the critical rays, some kind of peculiar behaviour of the distribution was expected at the points where the rays are tangent to 
the sphere. The explanation, detailed in the Appendix, lies in the generation process of the waves, discussed by Lighthill $(1978, \S 4.10)$ : in order to generate the waves, the source distribution is successively integrated in planes perpendicular to the direction of propagation of the phase, differentiated in this direction to one-halfth order, split into the sum of travelling waves moving in opposite directions, and the split waves sent diagonally along the rays. Each step of this process is susceptible to introducing singularities. One step, the splitting into travelling waves, amounts to adding to the source an out-of-phase component given by plus or minus its Hilbert transform.

For two-dimensional supercritical topographies, Pétrélis et al. (2006) and Balmforth \& Peacock (2009) used vertical and horizontal equivalent source distributions, respectively. The associated integral equations were solved numerically, and singular distributions were obtained. The singularities, however, were not located at the points where the critical rays are tangent to the topography, but at other points. A close inspection reveals that the origin of the singularities is numerical, and that it is linked with the use of vertical and horizontal source distributions, which typically exhibit discontinuities at the vertical and horizontal extremities of the topography, respectively, and with the discretization of the topography in terms of straight segments. We anticipate that if a contour source distribution is used instead, and the topography is discretized in terms of more complex boundary elements such as circular arcs or cubic splines (see e.g. Pozrikidis 2002, ch. 3), then the distribution will be regular.

Morris Flynn, Bruce Sutherland and Benjamin King are thanked for generously sharing their data. The data of Morris Flynn and Bruce Sutherland, in particular, initiated the present research. Inventive technical assistance by Pierre Carecchio and Joseph Virone is gratefully acknowledged. An anonymous referee is thanked for constructive criticism and insightful questioning. E.V.E. was supported by RFBRCNRS project No. 07-01-92212 and the 'Regular and Chaotic Hydrodynamics' European Research Network, and benefited from a visiting professorship at Joseph Fourier University from September-November 2007, during which the experiments were performed at LEGI. J.B.F. and the experimental devices built for this research were supported by grant FLOWINg of the French Research Agency. B.V. was supported by grants TOPOGI-3D and PIWO of the French Research Agency and grant LEFE/IDAO of INSU, and benefited from the hospitality of the Department of Mechanical and Aerospace Engineering of the University of California at San Diego, where part of the paper was written.

\section{Appendix. Generation process}

We consider steady inviscid far-field waves in the vertical plane represented in figure 1, combining a half-plane of the azimuthal angle $0 \leqslant \varphi_{0}<\pi$ on the right with the complementary half-plane of the angle $\varphi_{0}+\pi$ on the left. Following Lighthill $(1978, \S 4.10)$ and Voisin (2003), local Cartesian coordinates are introduced, denoted say by $\left(x_{ \pm}^{\prime}, y_{ \pm}^{\prime}, z_{ \pm}^{\prime}\right)$ and defined by

$$
\begin{gathered}
x=x_{ \pm}^{\prime} \cos \theta \cos \varphi_{0}-y_{ \pm}^{\prime} \sin \varphi_{0} \pm z_{ \pm}^{\prime} \sin \theta \cos \varphi_{0}, \\
y=x_{ \pm}^{\prime} \cos \theta \sin \varphi_{0}+y_{ \pm}^{\prime} \cos \varphi_{0} \pm z_{ \pm}^{\prime} \sin \theta \sin \varphi_{0}, \\
z=\mp x_{ \pm}^{\prime} \sin \theta+z_{ \pm}^{\prime} \cos \theta,
\end{gathered}
$$

such that $x_{ \pm}^{\prime}=x_{ \pm}$and $z_{ \pm}^{\prime}=z_{ \pm}$in the right half-plane and $x_{ \pm}^{\prime}=-x_{\mp}$ and $z_{ \pm}^{\prime}=z_{\mp}$ in the left half-plane, with $y_{ \pm}^{\prime}=0$ everywhere in the plane. Coordinates $\left(x_{+}^{\prime}, y_{+}^{\prime}, z_{+}^{\prime}\right)$ are 
used in the first and third quadrants, and coordinates $\left(x_{-}^{\prime}, y_{-}^{\prime}, z_{-}^{\prime}\right)$ in the second and fourth quadrants. Expression (2.9) of the velocity becomes

$$
\boldsymbol{u}_{0} \sim \pm \frac{\cot ^{1 / 2} \theta}{2^{5 / 2} \pi^{3 / 2}} \boldsymbol{e}_{z_{ \pm}^{\prime}} \frac{\exp [-\mathrm{i}(\omega t+\pi / 4)]}{\left|z_{ \pm}^{\prime}\right|^{1 / 2}} \int_{0}^{ \pm \infty \operatorname{sign} z}\left|k_{ \pm}^{\prime}\right|^{1 / 2} q_{ \pm}^{\prime}\left(k_{ \pm}^{\prime}, 0,0 \mid \varphi_{0}\right) \exp \left(\mathrm{i} k_{ \pm}^{\prime} x_{ \pm}^{\prime}\right) \mathrm{d} k_{ \pm}^{\prime},
$$

where $q_{ \pm}^{\prime}\left(x_{ \pm}^{\prime}, y_{ \pm}^{\prime}, z_{ \pm}^{\prime} \mid \varphi_{0}\right)=q(\boldsymbol{x})$ and $q_{ \pm}^{\prime}\left(k_{ \pm}^{\prime}, l_{ \pm}^{\prime}, m_{+}^{\prime} \mid \varphi_{0}\right)=q(\boldsymbol{k})$.

As pointed out by Lighthill $(1978, \S 4.10)$, this expression highlights the way the wave field is constructed. Fractional derivatives are involved, defined at order $\mu$, with $n \leqslant \operatorname{Re} \mu<n+1$, and for a function $f(x)$ of the spectrum $f(k)$, as

$$
\begin{aligned}
f^{(\mu)}(x)=\frac{1}{\Gamma(n+1-\mu)} \int_{-\infty}^{x} \frac{f^{(n+1)}(\xi)}{(x-\xi)^{\mu-n}} \mathrm{~d} \xi & \\
& =\frac{1}{2 \pi}\left(\mathrm{e}^{-\mathrm{i} \mu \pi / 2} \int_{-\infty}^{0}+\mathrm{e}^{\mathrm{i} \mu \pi / 2} \int_{0}^{+\infty}\right)|k|^{\mu} f(k) \exp (\mathrm{i} k x) \mathrm{d} k .
\end{aligned}
$$

First, the source is integrated in planes perpendicular to the $x_{+}^{\prime}$-direction of propagation of the phase (in other words, in planes tangent to the surfaces of constant phase along the rays), according to

$$
g\left(x_{ \pm}^{\prime} \mid \varphi_{0}\right)=\iint q_{ \pm}^{\prime}\left(x_{ \pm}^{\prime}, y_{ \pm}^{\prime}, z_{ \pm}^{\prime} \mid \varphi_{0}\right) \mathrm{d} y_{ \pm}^{\prime} \mathrm{d} z_{ \pm}^{\prime}=\frac{1}{2 \pi} \int_{-\infty}^{+\infty} q_{ \pm}^{\prime}\left(k_{ \pm}^{\prime}, 0,0 \mid \varphi_{0}\right) \exp \left(\mathrm{i} k_{ \pm}^{\prime} x_{ \pm}^{\prime}\right) \mathrm{d} k_{ \pm}^{\prime} .
$$

Then the integrated source is differentiated to one-halfth order, yielding

$$
g^{(1 / 2)}\left(x_{ \pm}^{\prime} \mid \varphi_{0}\right)=\left(\frac{\mathrm{e}^{\mathrm{i} \pi / 4}}{2 \pi} \int_{0}^{+\infty}+\frac{\mathrm{e}^{-\mathrm{i} \pi / 4}}{2 \pi} \int_{-\infty}^{0}\right)\left|k_{ \pm}^{\prime}\right|^{1 / 2} q_{ \pm}^{\prime}\left(k_{ \pm}^{\prime}, 0,0 \mid \varphi_{0}\right) \exp \left(\mathrm{i} k_{ \pm}^{\prime} x_{ \pm}^{\prime}\right) \mathrm{d} k_{ \pm}^{\prime} .
$$

Finally, the one-halfth derivative, viewed as a standing wave, is split into the sum of two travelling waves moving in opposite directions, and the waves are sent diagonally along the rays. One wave,

$$
g_{>}^{(1 / 2)}\left(x_{ \pm}^{\prime} \mid \varphi_{0}\right)=\frac{\mathrm{e}^{\mathrm{i} \pi / 4}}{2 \pi} \int_{0}^{+\infty}\left(k_{ \pm}^{\prime}\right)^{1 / 2} q_{ \pm}^{\prime}\left(k_{ \pm}^{\prime}, 0,0 \mid \varphi_{0}\right) \exp \left(\mathrm{i} k_{ \pm}^{\prime} x_{ \pm}^{\prime}\right) \mathrm{d} k_{ \pm}^{\prime},
$$

travels towards positive $x_{ \pm}^{\prime}$ and is sent upwards and downwards to the right, while the other wave,

$$
g_{<}^{(1 / 2)}\left(x_{ \pm}^{\prime} \mid \varphi_{0}\right)=\frac{\mathrm{e}^{-\mathrm{i} \pi / 4}}{2 \pi} \int_{-\infty}^{0}\left(-k_{ \pm}^{\prime}\right)^{1 / 2} q_{ \pm}^{\prime}\left(k_{ \pm}^{\prime}, 0,0 \mid \varphi_{0}\right) \exp \left(\mathrm{i} k_{ \pm}^{\prime} x_{ \pm}^{\prime}\right) \mathrm{d} k_{ \pm}^{\prime},
$$

travels towards negative $x_{ \pm}^{\prime}$ and is sent upwards and downwards to the left. These last two steps (differentiation to one-halfth order and splitting) may be applied in any order. Back in the original coordinates $\left(x_{ \pm}, \varphi, z_{ \pm}\right)$, the velocity becomes

$$
\boldsymbol{u}_{0} \sim \mp \mathrm{i} \frac{\cot ^{1 / 2} \theta}{2^{3 / 2} \pi^{1 / 2}} \boldsymbol{e}_{z_{ \pm}} \frac{\exp (-\mathrm{i} \omega t)}{\left|z_{ \pm}\right|^{1 / 2}} g_{>}^{(1 / 2)}\left(x_{ \pm} \mid \varphi\right) .
$$

We note that the splitting technique, based on one-sided Fourier transforms, is precisely that applied experimentally by Mercier et al. (2008) to separate the waves propagating along each arm of the St Andrew's cross for an oscillating cylinder. It is equivalent to adding to the forcing (once reduced to a function of the single variable $x_{+}^{\prime}$ ) an out-of-phase component given by plus or minus its Hilbert transform, a standard technique in signal processing to determine the analytic signal associated 
with a real signal (see e.g. Roddier 1978, §13.2), and a mechanism identified for internal tides over two-dimensional flat topographies by Bühler \& Muller (2007).

\section{A.1. Sphere}

In the two-dimensional case of an oscillating cylinder, no fractional derivative is involved and the algebraic singularity of the waves at the critical rays tangent to the cylinder arises from the first step in the process, namely the integration of the source along the rays, as mentioned by Ivanov (1989).

For the sphere, the singularity at the critical rays $\left|X_{ \pm}^{\prime}\right|=1$, where $X_{ \pm}^{\prime}=x_{ \pm}^{\prime} / a$, is only a discontinuity in the integrated source

$$
g\left(x_{ \pm}^{\prime} \mid \varphi_{0}\right)=2 \pi a\left[\frac{2 U \cos \theta}{1+B(\cos \theta)} \cos \varphi_{0} \mp \frac{W \sin \theta}{1-B(\cos \theta)}\right] X_{ \pm}^{\prime} H\left(1-\left|X_{ \pm}^{\prime}\right|\right),
$$

with $H(x)$ as the Heaviside step function. It is turned into an algebraic singularity by the next step, namely the differentiation to one-halfth order, according to

$$
\begin{aligned}
g^{(1 / 2)}\left(x_{ \pm}^{\prime} \mid \varphi_{0}\right)=-2^{3 / 2}(\pi a)^{1 / 2}\left[\frac{2 U \cos \theta}{1+B(\cos \theta)} \cos \varphi_{0} \mp \frac{W \sin \theta}{1-B(\cos \theta)}\right] \\
\quad \times \begin{cases}\frac{\left[X_{ \pm}^{\prime}-\left(X_{ \pm}^{\prime 2}-1\right)^{1 / 2}\right]^{3 / 2}}{\left(X_{ \pm}^{\prime 2}-1\right)^{1 / 2}} H\left(X_{ \pm}^{\prime}\right) & \left(\left|X_{ \pm}^{\prime}\right|>1\right), \\
-\frac{\sin \left(\frac{3}{2} \arccos X_{ \pm}^{\prime}\right)}{\left(1-X_{ \pm}^{\prime 2}\right)^{1 / 2}} & \left(\left|X_{ \pm}^{\prime}\right|<1\right) .\end{cases}
\end{aligned}
$$

Subsequent splitting into travelling waves yields

$$
\begin{aligned}
g_{>}^{(1 / 2)}\left(x_{ \pm}^{\prime} \mid \varphi_{0}\right)=-(2 \pi a)^{1 / 2}\left[\frac{2 U \cos \theta}{1+B(\cos \theta)} \cos \varphi_{0}\right. & \left.\mp \frac{W \sin \theta}{1-B(\cos \theta)}\right] \\
& \times \frac{\exp \left[-\frac{3}{2} \operatorname{arccosh}\left(X_{ \pm}^{\prime}+\mathrm{i} 0\right)\right]}{\left[\left(X_{ \pm}^{\prime}+\mathrm{i} 0\right)^{2}-1\right]^{1 / 2}},
\end{aligned}
$$

with $g_{<}^{(1 / 2)}$ as the complex conjugate of $g_{>}^{(1 / 2)}$. Each step of the process is illustrated in figure 30 . The resulting velocity, which may be written

$$
\begin{aligned}
& \boldsymbol{u}_{0} \sim \mathrm{i} \frac{\cot ^{1 / 2} \theta}{2^{5 / 2}}\left[ \pm \frac{2 U \cos \theta}{1+B(\cos \theta)} \cos \varphi\right.\left.-\frac{W \sin \theta}{1-B(\cos \theta)}\right] \boldsymbol{e}_{z_{ \pm}} \frac{\exp (-\mathrm{i} \omega t)}{\left|Z_{ \pm}\right|^{1 / 2}} \\
& \times \frac{\left[\left(X_{ \pm}+1+\mathrm{i} 0\right)^{1 / 2}-\left(X_{ \pm}-1+\mathrm{i} 0\right)^{1 / 2}\right]^{3}}{\left(X_{ \pm}+1+\mathrm{i} 0\right)^{1 / 2}\left(X_{ \pm}-1+\mathrm{i} 0\right)^{1 / 2}},
\end{aligned}
$$

consistent with (3.6), has a bimodal profile with algebraic singularities at the critical rays, a specific property of supercritical wave generators.

\section{A.2. Flat topographies}

In contrast, for the flat topographies considered in $\S 7$, necessarily subcritical, unimodal profiles are expected. We verify this for the witch of Agnesi, which becomes, upon integration,

$$
g\left(x_{+}^{\prime} \mid \varphi_{0}\right)=8 h U \frac{\cos \varphi_{0}}{\cos \theta} \frac{X_{+}^{\prime}}{\left(1+X_{+}^{\prime 2}\right)^{2}},
$$



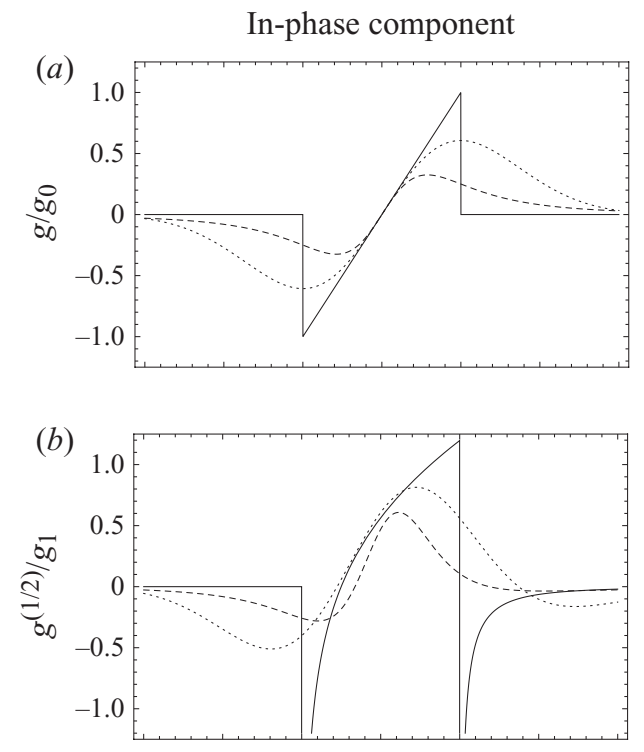

Out-of-phase component
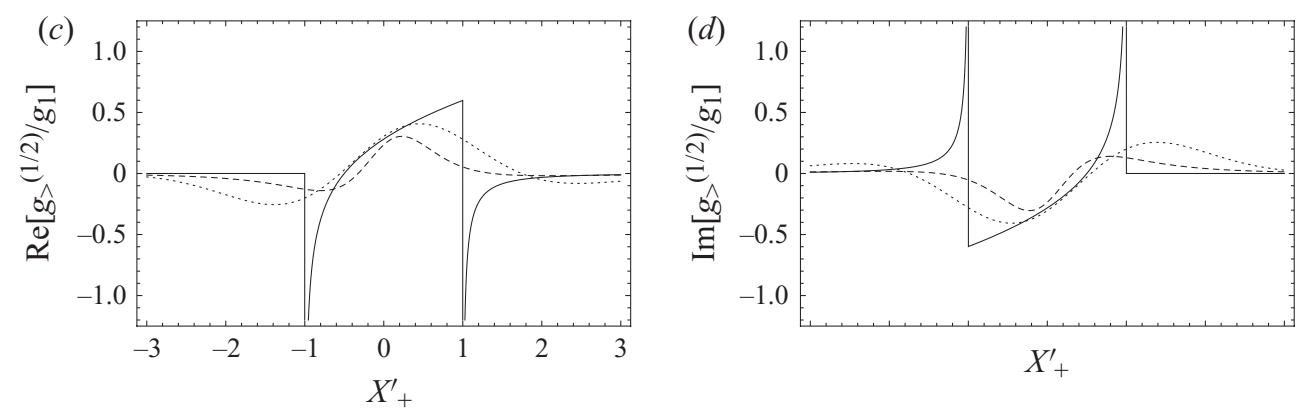

Amplitude

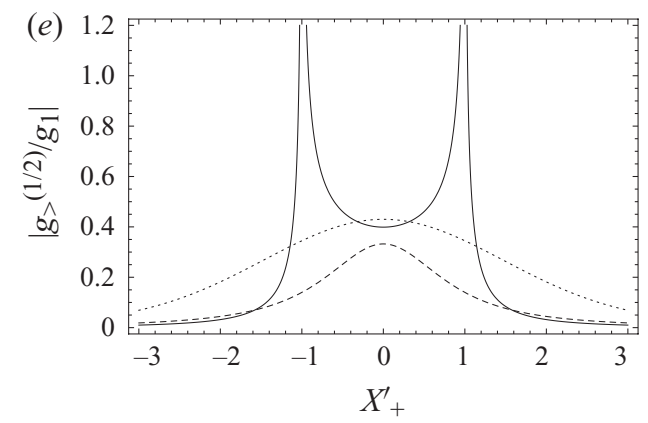

FIgURE 30. Transformation of the source function into the wave profile, for the sphere (solid lines), the witch of Agnesi (dashed lines) and the Gaussian bump (dotted lines), in three steps: integration perpendicular to the direction of propagation of the phase, yielding profile $(a)$; differentiation to one-halfth order along this direction, yielding profile $(b)$; splitting into travelling waves propagating in opposite directions, yielding the in-phase profile $(c)$, out-of-phase profile $(d)$ and amplitude profile $(e)$. The normalization constant $g_{0}$ has been chosen such that the integrated sources are $X_{+}^{\prime} H\left(1-\left|X_{+}^{\prime}\right|\right), X_{+}^{\prime} /\left(1+X_{+}^{\prime 2}\right)^{2}$ and $X_{+}^{\prime} \exp \left(-X_{+}^{\prime 2} / 2\right)$ for the sphere, the witch of Agnesi and the Gaussian bump, respectively, and $g_{1}$ has been taken as $g_{0} / a^{1 / 2}$ for the sphere and $g_{0} /(\ell \cos \theta)^{1 / 2}$ otherwise. 
differentiation to one-halfth order,

$$
g^{(1 / 2)}\left(x_{+}^{\prime} \mid \varphi_{0}\right)=3\left(\frac{\pi}{\ell}\right)^{1 / 2} h U \frac{\cos \varphi_{0}}{\cos ^{3 / 2} \theta} \frac{\cos \left(\frac{5}{2} \arctan X_{+}^{\prime}-\frac{\pi}{4}\right)}{\left(1+X_{+}^{\prime 2}\right)^{5 / 4}},
$$

and splitting,

$$
g_{>}^{(1 / 2)}\left(x_{+}^{\prime} \mid \varphi_{0}\right)=\frac{3}{2}\left(\frac{\pi}{\ell}\right)^{1 / 2} h U \frac{\cos \varphi_{0}}{\cos ^{3 / 2} \theta} \frac{\exp \left[\mathrm{i}\left(\frac{5}{2} \arctan X_{+}^{\prime}-\frac{\pi}{4}\right)\right]}{\left(1+X_{+}^{\prime 2}\right)^{5 / 4}} .
$$

It bears close similarity to the examples considered by Lighthill $(1978, \S 4.10)$. The velocity profile, described by

$$
\boldsymbol{u}_{0} \sim \frac{3}{2} \frac{h}{\ell} \frac{U \cos \varphi}{\sin ^{1 / 2} \theta \cos ^{3 / 2} \theta} \boldsymbol{e}_{z+} \frac{\exp [-\mathrm{i}(\omega t+3 \pi / 4)]}{Z_{+}^{1 / 2}} \frac{1}{\left(1-\mathrm{i} X_{+}\right)^{5 / 2}},
$$

is plotted in figure 30 and is indeed unimodal.

The Gaussian bump yields more complicated expressions, upon integration,

$$
g\left(x_{+}^{\prime} \mid \varphi_{0}\right)=2^{3 / 2} \pi^{1 / 2} h U \frac{\cos \varphi_{0}}{\cos \theta} X_{+}^{\prime} \exp \left(-\frac{X_{+}^{\prime 2}}{2}\right),
$$

differentiation to one-halfth order,

$$
g^{(1 / 2)}\left(x_{+}^{\prime} \mid \varphi_{0}\right)=-2^{3 / 2}\left(\frac{\pi}{\ell}\right)^{1 / 2} h U \frac{\cos \varphi_{0}}{\cos ^{3 / 2} \theta} \exp \left(-\frac{X_{+}^{\prime 2}}{4}\right) D_{3 / 2}\left(-X_{+}^{\prime}\right),
$$

and splitting,

$$
g_{>}^{(1 / 2)}\left(x_{+}^{\prime} \mid \varphi_{0}\right)=\frac{3}{2} \mathrm{e}^{-\mathrm{i} \pi / 4}\left(\frac{\pi}{\ell}\right)^{1 / 2} h U \frac{\cos \varphi_{0}}{\cos ^{3 / 2} \theta} \exp \left(-\frac{X_{+}^{\prime 2}}{4}\right) D_{-5 / 2}\left(-\mathrm{i} X_{+}^{\prime}\right),
$$

involving the parabolic cylinder function $D_{\mu}(x)$ related to the more familiar modified Bessel function $I_{\mu}(x)$ by

$$
\begin{gathered}
D_{3 / 2}(-x)=-\frac{\pi^{1 / 2} x^{5 / 2}}{8}\left(3 I_{3 / 4}-2 I_{-1 / 4}-I_{-5 / 4}+3 I_{-3 / 4}-2 I_{1 / 4}-I_{5 / 4}\right)\left(\frac{x^{2}}{4}\right), \\
D_{-5 / 2}(-\mathrm{i} x)=\frac{\pi^{1 / 2} x^{5 / 2}}{6}\left[3 I_{3 / 4}-2 I_{-1 / 4}-I_{-5 / 4}+\mathrm{i}\left(3 I_{-3 / 4}-2 I_{1 / 4}-I_{5 / 4}\right)\right]\left(\frac{x^{2}}{4}\right) .
\end{gathered}
$$

However, the velocity profile, described by

$$
\boldsymbol{u}_{0} \sim \frac{3}{2^{5 / 2}} \frac{h}{\ell} \frac{U \cos \varphi}{\sin ^{1 / 2} \theta \cos ^{3 / 2} \theta} \boldsymbol{e}_{z+} \frac{\exp [-\mathrm{i}(\omega t+3 \pi / 4)]}{Z_{+}^{1 / 2}} \exp \left(-\frac{X_{+}^{2}}{4}\right) D_{-5 / 2}\left(-\mathrm{i} X_{+}\right),
$$

and plotted in figure 30 , is similar. It is consistent with the theoretical predictions by Bühler \& Muller (2007), who did not use the far-field approximation, and with the experimental measurements by Peacock et al. (2008), who considered a twodimensional Gaussian bump of non-small $h / \ell \approx 0.7$. 


\section{REFERENCES}

Appleby, J. C. \& Crighton, D. G. 1986 Non-Boussinesq effects in the diffraction of internal waves from an oscillating cylinder. Q. J. Mech. Appl. Math. 39, 209-231.

Appleby, J. C. \& Crighton, D. G. 1987 Internal gravity waves generated by oscillations of a sphere. J. Fluid Mech. 183, 439-450.

BAINES, P. G. \& FANG, X.-H. 1985 Internal tide generation at a continental shelf/slope junction: a comparison between theory and a laboratory experiment. Dyn. Atmos. Oceans 9, 297-314.

Balmforth, N. J. \& Peacock, T. 2009 Tidal conversion by supercritical topography. J. Phys. Oceanogr. 39, 1965-1974.

Batchelor, G. K. 1967 An Introduction to Fluid Dynamics. Cambridge University Press.

BeLL, T. H. $1975 a$ Lee waves in stratified flows with simple harmonic time dependence. J. Fluid Mech. 67, 705-722.

BeLL, T. H. $1975 b$ Topographically generated internal waves in the open ocean. J. Geophys. Res. 80, 320-327.

Bonnier, M., Bonneton, P. \& Eiff, O. 1998 Far-wake of a sphere in a stably stratified fluid: characterization of the vortex structures. Appl. Sci. Res. 59, 269-281.

BüHLER, O. \& Muller, C. J. 2007 Instability and focusing of internal tides in the deep ocean. J. Fluid Mech. 588, 1-28.

Cerasoli, C. P. 1978 Experiments on buoyant-parcel motion and the generation of internal gravity waves. J. Fluid Mech. 86, 247-271.

Chashechkin, Y. D. 2007 Visualization of singular components of periodic motions in a continuously stratified fluid. J. Vis. 10, 17-20.

Chashechinin, Y. D., Kistovich, Y. V. \& Smirnov, S. A. 2001 Linear generation theory of 2D and 3D periodic internal waves in a viscous stratified fluid. Environmetrics 12, 57-80.

CHASHEChKIN, Y. D. \& PRIKHOD'Ko, Y. V. 2007 Regular and singular flow components for stimulated and free oscillations of a sphere in continuously stratified liquid. Dokl. Phys. 52, 261-265.

Clark, H. A. \& Sutherland, B. R. 2009 Schlieren measurements of internal waves in nonBoussinesq fluids. Exp. Fluids 47, 183-193.

Dalziel, S. B., Hughes, G. O. \& Sutherland, B. R. 2000 Whole-field density measurements by 'synthetic schlieren'. Exp. Fluids 28, 322-335.

Davis, A. M. J. \& Llewellyn Smith, S. G. 2010 Tangential oscillations of a circular disk in a viscous stratified fluid. J. Fluid Mech. 656, 342-359.

DÉCAmp, S., Kozack, C. \& Sutherland, B. R. 2008 Three-dimensional schlieren measurements using inverse tomography. Exp. Fluids 44, 747-758.

Di Lorenzo, E., Young, W. R. \& Llewellyn Smith, S. 2006 Numerical and analytical estimates of $M_{2}$ tidal conversion at steep oceanic ridges. J. Phys. Oceanogr. 36, 1072-1084.

Echeverri, P. E, Flynn, M. R., Winters, K. B. \& Peacock, T. 2009 Low-mode internal tide generation by topography: an experimental and numerical investigation. J. Fluid Mech. 636, 91-108.

ERMANYUK, E. V. 2002 The rule of affine similitude for the force coefficients of a body oscillating in a uniformly stratified fluid. Exp. Fluids 32, 242-251.

Ermanyuk, E. V., Flór, J.-B. \& Voisin, B. 2010 Spatial structure of first and higher harmonic internal waves from a horizontally oscillating sphere. J. Fluid Mech. (submitted).

ERmanYuK, E. V. \& GaVRILOV, N. V. 2002 Force on a body in a continuously stratified fluid. Part 1. Circular cylinder. J. Fluid Mech. 451, 421-443.

Ermanyuk, E. V. \& GaVriLov, N. V. 2003 Force on a body in a continuously stratified fluid. Part 2. Sphere. J. Fluid Mech. 494, 33-50.

Ermanyuk, E. V. \& Gavrilov, N. V. 2005 Duration of transient processes in the formation of internal-wave beams. Dokl. Phys. 50, 548-550.

Ermanyuk, E. V. \& GaVRilov, N. V. 2008 On internal waves generated by large-amplitude circular and rectilinear oscillations of a circular cylinder in a uniformly stratified fluid. J. Fluid Mech. 613, 329-356.

FERRARI, R. \& WUNSCH, C. 2009 Ocean circulation kinetic energy: reservoirs, sources, and sinks. Annu. Rev. Fluid Mech. 41, 253-282.

Flór, J.-B., Bush, J. W. M. \& Ungarish, M. 2004 An experimental investigation of spin-up from rest of a stratified fluid. Geophys. Astrophys. Fluid Dyn. 98, 277-296. 
FlóR, J.-B., Ungarish, M. \& Bush, J. W. M. 2002 Spin-up from rest in a stratified fluid: boundary flows. J. Fluid Mech. 472, 51-82.

FlynN, M. R., ONU, K. \& Sutherland, B. R. 2003 Internal wave excitation by a vertically oscillating sphere. J. Fluid Mech. 494, 65-93.

Fortuin, J. M. H. 1960 Theory and application of two supplementary methods of constructing density gradient columns. J. Polym. Sci. 44, 505-515.

GARretT, C. 2003 Internal tides and ocean mixing. Science 301, 1858-1859.

Garrett, C. \& Kunze, E. 2007 Internal tide generation in the deep ocean. Annu. Rev. Fluid Mech. 39, 57-87.

Gordon, D. \& Stevenson, T. N. 1972 Viscous effects in a vertically propagating internal wave. J. Fluid Mech. 56, 629-639.

Gorodtsov, V. A. \& TeOdorovich, E. V. 1982 Study of internal waves in the case of rapid horizontal motion of cylinders and spheres. Fluid Dyn. 17, 893-898.

Gorodtsov, V. A. \& Teodorovich, E. V. 1986 Energy characteristics of harmonic internal wave generators. J. Appl. Mech. Tech. Phys. 27, 523-529.

GöRTLER, H. 1943 On an oscillatory phenomenon in fluids of a stable distribution of density. Z. Angew. Math. Mech. 23, 65-71 (in German).

Gostiaux, L. \& Dauxois, T. 2007 Laboratory experiments on the generation of internal tidal beams over steep slopes. Phys. Fluids 19, 028102.

Hall, P. 1984 On the stability of the unsteady boundary layer on a cylinder oscillating transversely in a viscous fluid. J. Fluid Mech. 146, 347-367.

Hendershott, M. C. 1969 Impulsively started oscillations in a rotating stratified fluid. J. Fluid Mech. 36, 513-527.

Hopfinger, E. J., Flór, J.-B., Chomaz, J.-M. \& Bonneton, P. 1991 Internal waves generated by a moving sphere and its wake in a stratified fluid. Exp. Fluids 11, 255-261.

Hurley, D. G. 1969 The emission of internal waves by vibrating cylinders. J. Fluid Mech. 36, $657-672$.

Hurley, D. G. 1972 A general method for solving steady-state internal gravity wave problems. J. Fluid Mech. 56, 721-740.

Hurley, D. G. 1997 The generation of internal waves by vibrating elliptic cylinders. Part 1. Inviscid solution. J. Fluid Mech. 351, 105-118.

Hurley, D. G. \& Hood, M. J. 2001 The generation of internal waves by vibrating elliptic cylinders. Part 3. Angular oscillations and comparison of theory with recent experimental observations. J. Fluid Mech. 433, 61-75.

Hurley, D. G. \& Keady, G. 1997 The generation of internal waves by vibrating elliptic cylinders. Part 2. Approximate viscous solution. J. Fluid Mech. 351, 119-138.

IL'INYKh, Y. S., Smirnov, S. A. \& Chashechin, Y. D. 1999 Excitation of harmonic internal waves in a viscous continuously stratified liquid. Fluid Dyn. 34, 890-895.

IvANOV, A. V. 1988 Visualization and measurements of density inhomogeneities in a density stratified fluid using the Moiré fringe method. Preprint No. 189, Institute of Applied Physics, Academy of Sciences of the USSR, Gorkii (in Russian).

Ivanov, A. V. 1989 Generation of internal waves by an oscillating source. Izv. Atmos. Ocean. Phys. 25, 61-64.

Ivey, G. N., Winters, K. B. \& KosefF, J. R. 2008 Density stratification, turbulence, but how much mixing? Annu. Rev. Fluid Mech. 40, 169-184.

King, B., Zhang, H. P. \& Swinney, H. L. 2009 Tidal flow over three-dimensional topography in a stratified fluid. Phys. Fluids 21, 116601.

Kistovich, Y. V. \& Chashechkin, Y. D. 1999a Generation of monochromatic internal waves in a viscous fluid. J. Appl. Mech. Tech. Phys. 40, 1020-1028.

Kistovich, Y. V. \& ChashechKin, Y. D. $1999 b$ An exact solution of a linearized problem of the radiation of monochromatic internal waves in a viscous fluid. J. Appl. Math. Mech. 63, 587-594.

Kunze, E. \& Llewellyn Smith, S. G. 2004 The role of small-scale topography in turbulent mixing of the global ocean. Oceanography 17, 55-64.

Lighthill, J. 1978 Waves in Fluids. Cambridge University Press.

Lin, Q., Boyer, D. L. \& Fernando, H. J. S. 1994 Flows generated by the periodic horizontal oscillations of a sphere in a linearly stratified fluid. J. Fluid Mech. 263, 245-270. 
Llewellyn Smith, S. G. \& Young, W. R. 2002 Conversion of the barotropic tide. J. Phys. Oceanogr. 32, 1554-1566.

Llewellyn Smith, S. G. \& Young, W. R. 2003 Tidal conversion at a very steep ridge. J. Fluid Mech. 495, 175-191.

Makarov, S. A., Neklyudov, V. I. \& Chashechkin, Y. D. 1990 Spatial structure of two-dimensional monochromatic internal-wave beams in an exponentially stratified liquid. Izv. Atmos. Ocean. Phys. 26, 548-554.

Makhortykh, S. A. \& RYBak, S. A. 1990 Effect of the near field of a point source on the generation of internal waves. Izv. Atmos. Ocean. Phys. 26, 194-198.

McLaren, T. I., Pierce, A. D., Fohl, T. \& Murphy, B. L. 1973 An investigation of internal gravity waves generated by a buoyantly rising fluid in a stratified medium. J. Fluid Mech. 57, 229-240.

Mercier, M. J., Garnier, N. B. \& Dauxois, T. 2008 Reflection and diffraction of internal waves analyzed with the Hilbert transform. Phys. Fluids 20, 086601.

Merzkirch, W. \& Peters, F. 1992 Optical visualization of internal gravity waves in stratified fluid. Opt. Laser. Engng 16, 411-425.

Mowbray, D. E. \& Rarity, B. S. H. 1967 A theoretical and experimental investigation of the phase configuration of internal waves of small amplitude in a density stratified liquid. J. Fluid Mech. 28, $1-16$.

NycANDER, J. 2005 Generation of internal waves in the deep ocean by tides. J. Geophys. Res. 110, C10028.

NyCANDER, J. 2006 Tidal generation of internal waves from a periodic array of steep ridges. J. Fluid Mech. 567, 415-432.

Onu, K., Flynn, M. R. \& Sutherland, B. R. 2003 Schlieren measurement of axisymmetric internal wave amplitudes. Exp. Fluids 35, 24-31.

Oster, G. 1965 Density gradients. Sci. Am. 213, 70-76.

Oster, G. \& Yамамото, M. 1963 Density gradient techniques. Chem. Rev. 63, 257-268.

Отто, S. R. 1992 On stability of the flow around an oscillating sphere. J. Fluid Mech. 239, 47-63.

Peacock, T., Echeverri, P. \& Balmforth, N. J. 2008 An experimental investigation of internal tide generation by two-dimensional topography. J. Phys. Oceanogr. 38, 235-242.

Peacock, T. \& Weidman, P. 2005 The effect of rotation on conical wave beams in a stratified fluid. Exp. Fluids 39, 32-37.

Peters, F. 1985 Schlieren interferometry applied to a gravity wave in a density-stratified liquid. Exp. Fluids 3, 261-269.

Pétrélis, F., Llewellyn Smith, S. \& Young, W. R. 2006 Tidal conversion at a submarine ridge. J. Phys. Oceanogr. 36, 1053-1071.

Pozrikidis, C. 1992 Boundary Integral and Singularity Methods for Linearized Viscous Flow. Cambridge University Press.

Pozrikidis, C. 2002 A Practical Guide to Boundary Element Methods with the Software Library BEMLIB. Chapman \& Hall/CRC.

Riley, J. J., Metcalfe, R. W. \& Weissman, M. A. 1981 Direct numerical simulations of homogeneous turbulence in density-stratified fluids. AIP Conf. Proc. 76, 79-112.

Riley, N. 2001 Steady streaming. Annu. Rev. Fluid Mech. 33, 43-65.

RodDier, F. 1978 Distributions et Transformation de Fourier. McGraw-Hill.

Saint-Exupéry, A. De 1946 Le Petit Prince. Gallimard.

SAKAI, S. 1990 Visualization of internal gravity wave by Moiré method. Trans. Vis. Soc. Japan 10, 65-68 (in Japanese).

SARma, L. V. K. V. \& Krishna, D. V. 1972 Oscillation of axisymmetric bodies in a stratified fluid. Zastosow. Matem. 13, 109-121.

Simakov, S. T. 1994 Formation of singularities of limiting amplitude in a density stratified fluid disturbed by an extended monochromatic forcing. Wave Motion 19, 11-27.

St. Laurent, L. \& Garrett, C. 2002 The role of internal tides in mixing the deep ocean. J. Phys. Oceanogr. 32, 2882-2899.

Sutherland, B. R., Dalziel, S. B., Hughes, G. O. \& Linden, P. F. 1999 Visualization and measurement of internal waves by 'synthetic schlieren'. Part 1. Vertically oscillating cylinder. J. Fluid Mech. 390, 93-126. 
Sutherland, B. R., Flynn, M. R. \& ONU, K. 2003 Schlieren visualisation and measurement of axisymmetric disturbances. Nonlinear Process. Geophys. 10, 303-309.

Sutherland, B. R., Hughes, G. O., Dalziel, S. B. \& Linden, P. F. 2000 Internal waves revisited. Dyn. Atmos. Oceans 31, 209-232.

Sutherland, B. R. \& Linden, P. F. 2002 Internal wave excitation by a vertically oscillating elliptical cylinder. Phys. Fluids 14, 721-731.

Thomas, L. P., Marino, B. M. \& Dalziel, S. B. 2009 Synthetic schlieren: determination of the density gradient generated by internal waves propagating in a stratified fluid. J. Phys. Conf. Ser. 166, 012007.

Thomas, N. H. \& Stevenson, T. N. 1972 A similarity solution for viscous internal waves. J. Fluid Mech. 54, 495-506.

Vlasenko, V., Stashchuk, N. \& Hutter, K. 2005 Baroclinic Tides: Theoretical Modeling and Observational Evidence. Cambridge University Press.

VoIsIn, B. 1991 Internal wave generation in uniformly stratified fluids. Part 1. Green's function and point sources. J. Fluid Mech. 231, 439-480.

Voisin, B. 2003 Limit states of internal wave beams. J. Fluid Mech. 496, 243-293.

Voisin, B. 2007 Lee waves from a sphere in a stratified flow. J. Fluid Mech. 574, 273-315.

VoIsIN, B. $2010 a$ Oscillating bodies in density-stratified fluids. Part 1. Wave field. In preparation.

VoIsIN, B. $2010 b$ Oscillating bodies in density-stratified fluids. Part 2. Added mass. In preparation.

VoIsIN, B. 2010c Transition regimes of internal wave beams. In preparation.

Westerweel, J. 1997 Fundamentals of digital particle image velocimetry. Meas. Sci. Technol. 8, $1379-1392$.

WU, J. 1969 Mixed region collapse with internal wave generation in a density-stratified medium. J. Fluid Mech. 35, 531-544.

WunsCh, C. \& Ferrari, R. 2004 Vertical mixing, energy, and the general circulation of the oceans. Annu. Rev. Fluid Mech. 36, 281-314.

Xu, Y., Boyer, D. L., Fernando, H. J. S. \& Zhang, X. 1997 Motion fields generated by the oscillatory motion of a circular cylinder in a linearly stratified fluid. Expl Thermal Fluid Sci. 14, 277-296.

Zhang, H. P., King, B. \& Swinney, H. L. 2007 Experimental study of internal gravity waves generated by supercritical topography. Phys. Fluids 19, 096602.

Zhang, H. P., King, B. \& Swinney, H. L. 2008 Resonant generation of internal waves on a model continental slope. Phys. Rev. Lett. 100, 244504. 